\title{
Dense gas in the Galactic central molecular zone is warm and heated by turbulence ${ }^{\star}$
}

\author{
Adam Ginsburg ${ }^{1}$, Christian Henkel ${ }^{2,3}$, Yiping Ao ${ }^{4,5}$, Denise Riquelme ${ }^{2}$, Jens Kauffmann ${ }^{2}$, Thushara Pillai ${ }^{2}$, \\ Elisabeth A. C. Mills ${ }^{6}$, Miguel A. Requena-Torres ${ }^{2}$, Katharina Immer $^{1}$, Leonardo Testi ${ }^{1}$, Juergen Ott ${ }^{6}$, John Bally ${ }^{7}$, \\ Cara Battersby $^{8}$, Jeremy Darling ${ }^{7}$, Susanne Aalto ${ }^{9}$, Thomas Stanke ${ }^{1}$, Sarah Kendrew ${ }^{10}$, J. M. Diederik Kruijssen ${ }^{11}$, \\ Steven Longmore ${ }^{12}$, James Dale ${ }^{13}$, Rolf Guesten ${ }^{2}$, and Karl M. Menten ${ }^{2}$ \\ ${ }^{1}$ European Southern Observatory, Karl-Schwarzschild-Strasse 2, 85748 Garching bei München, Germany \\ e-mail: Adam.Ginsburg@eso.org \\ 2 Max Planck Institute for Radio Astronomy, auf dem Hugel, 53121 Bonn, Germany \\ 3 Astron. Dept., King Abdulaziz University, PO Box 80203, 21589 Jeddah, Saudi Arabia \\ ${ }^{4}$ National Astronomical Observatory of Japan, 2-21-1 Osawa, Mitaka, 181-8588 Tokyo, Japan \\ 5 Purple Mountain Observatory, Chinese Academy of Sciences, 210008 Nanjing, PR China \\ ${ }^{6}$ National Radio Astronomy Observatory, Socorro, NM 87801, USA \\ 7 CASA, University of Colorado, 389-UCB, Boulder, CO 80309, USA \\ 8 Harvard-Smithsonian Center for Astrophysics, 60 Garden Street, Cambridge, MA 02138, USA \\ 9 Department of Earth and Space Sciences, Chalmers University of Technology, 41258 Gôteborg, Sweden \\ ${ }^{10}$ Department of Astrophysics, The Denys Wilkinson Building, Keble Road, Oxford OX1 3RH, UK \\ 11 Max-Planck Institut für Astrophysik, Karl-Schwarzschild-Straße 1, 85748 Garching, Germany \\ 12 Astrophysics Research Institute, Liverpool John Moores University, IC2, Liverpool Science Park, 146 Brownlow Hill, \\ Liverpool L3 5RF, UK \\ 13 University Observatory Munich, Scheinerstr. 1, 81679 München, Germany
}

Received 15 March 2015 / Accepted 16 August 2015

\begin{abstract}
Context. The Galactic center is the closest region where we can study star formation under extreme physical conditions like those in high-redshift galaxies.

Aims. We measure the temperature of the dense gas in the central molecular zone (CMZ) and examine what drives it.

Methods. We mapped the inner $300 \mathrm{pc}$ of the $\mathrm{CMZ}$ in the temperature-sensitive $J=3-2$ para-formaldehyde (p- $\left.\mathrm{H}_{2} \mathrm{CO}\right)$ transitions. We used the $3_{2,1}-2_{2,0} / 3_{0,3}-2_{0,2}$ line ratio to determine the gas temperature in $n \sim 10^{4}-10^{5} \mathrm{~cm}^{-3}$ gas. We have produced temperature maps and cubes with $30^{\prime \prime}$ and $1 \mathrm{~km} \mathrm{~s}^{-1}$ resolution and published all data in FITS form.

Results. Dense gas temperatures in the Galactic center range from $\sim 60 \mathrm{~K}$ to $>100 \mathrm{~K}$ in selected regions. The highest gas temperatures $T_{\mathrm{G}}>100 \mathrm{~K}$ are observed around the Sgr B2 cores, in the extended Sgr B2 cloud, the $20 \mathrm{~km} \mathrm{~s}^{-1}$ and $50 \mathrm{~km} \mathrm{~s}^{-1}$ clouds, and in "The Brick" (G0.253+0.016). We infer an upper limit on the cosmic ray ionization rate $\zeta_{\mathrm{CR}}<10^{-14} \mathrm{~s}^{-1}$.

Conclusions. The dense molecular gas temperature of the region around our Galactic center is similar to values found in the central regions of other galaxies, in particular starburst systems. The gas temperature is uniformly higher than the dust temperature, confirming that dust is a coolant in the dense gas. Turbulent heating can readily explain the observed temperatures given the observed line widths. Cosmic rays cannot explain the observed variation in gas temperatures, so CMZ dense gas temperatures are not dominated by cosmic ray heating. The gas temperatures previously observed to be high in the inner $\sim 75 \mathrm{pc}$ are confirmed to be high in the entire CMZ.
\end{abstract}

Key words. Galaxy: center - ISM: molecules - ISM: structure - Galaxy: nucleus - cosmic rays - ISM: clouds

\section{Introduction}

The central region of our Galaxy, the central molecular zone (CMZ), is the nearest location in which we can study star formation in an extreme environment with pressure, turbulent Mach number, and gas temperature much higher than in local star-forming regions (Morris \& Serabyn 1996). While there have been great leaps in our understanding of star formation in

$\star$ The data can be accessed from doi: 10.7910/DVN/27601 and are available at the CDS via anonymous ftp to cdsarc.u-strasbg.fr $(130.79 .128 .5)$ or via

http://cdsarc.u-strasbg.fr/viz-bin/qcat?J/A+A/586/A50 local molecular clouds in the past decade, there remain many unanswered questions about how star formation changes as gas becomes denser, more opaque, and more turbulent, as it most likely was in galaxies at the peak of cosmic star formation (Kruijssen \& Longmore 2013).

The Galactic CMZ has attracted a great deal of attention recently because it has a much higher ratio of dense gas mass to star formation tracers than the rest of the galaxy (Longmore et al. 2013b, 2012, 2013a; Kruijssen et al. 2014; Yusef-Zadeh et al. 2009; Immer et al. 2012). The star formation rate in this region therefore appears to be suppressed relative to the expectations from nearby star-forming regions and nearby galaxy disks, 
where the star formation timescale decreases with increasing gas surface density (Kennicutt 1998; Kennicutt \& Evans 2012; Leroy et al. 2013; Heiderman et al. 2010). Within the CMZ, the central $\sim 100 \mathrm{pc}^{1}$ "ring" including the dust ridge (Lis et al. 1991; Sofue 1995; Molinari et al. 2011) contains most of the dense gas. Longmore et al. (2013b) recently proposed that the clouds along the "ring" (which is more accurately described as a stream; Kruijssen et al. 2015) represent a time-ordered path from starless to star-forming clouds, from "The Brick" (G0.253+0.016, cloud a) through the lettered dust ridge clouds (clouds b, c, d, e, f Lis et al. 1999), ending at Sgr B2. In principle, this would allow us to study the time evolution of protocluster clumps given an orbital model to describe their motion (Kruijssen et al. 2015).

The thermal properties of this gas are a crucial component for understanding the conditions governing both star formation and interstellar chemistry. The gas temperature sets the sound speed in the gas and therefore the Mach number within a turbulent medium. It also governs the thermal Jeans mass. Regardless of whether turbulence or gravity controls the fragmentation scale, the gas temperature is clearly important.

Prior gas temperature measurements in the $\mathrm{CMZ}$ primarily used the popular ammonia metastable inversion transition thermometer $\left(\mathrm{NH}_{3}(1,1)-(7,7)\right.$; Güsten et al. 1981; Mauersberger et al. 1986; Hüttemeister et al. 1993; Ott et al. 2014), which is sensitive to moderate density gas $\left(n\left(\mathrm{H}_{2}\right) \sim 10^{3}-10^{4} \mathrm{~cm}^{-3}\right.$; Shirley 2015). While this thermometer is generally reliable in cold regions (e.g., the $(1-1) /(2-2)$ ratio is accurate to $T_{\mathrm{G}} \lesssim$ $40 \mathrm{~K}$ ), the population of the higher energy states may be affected by formation heating, a mechanism recently discovered to affect $\mathrm{H}_{3} \mathrm{O}+$ in the $\mathrm{CMZ}$ and suggested to affect $\mathrm{NH}_{3}$, in which the excess energy from molecule formation leaves a significant fraction of the molecules in a rotationally excited state (Lis et al. 2014), so confirmation of the high temperatures seen in $\mathrm{NH}_{3}$ (Mills \& Morris 2013) can be used to assess the significance of that mechanism. Ao et al. (2013) used the paraformaldehyde ( $\left.\mathrm{p}-\mathrm{H}_{2} \mathrm{CO}\right) 218 \mathrm{GHz}$ thermometer (Mangum \& Wootten 1993), which is sensitive to denser $\left(n \sim 10^{4}-10^{5} \mathrm{~cm}^{-3}\right)$ and hotter $\left(T_{\mathrm{G}}>20 \mathrm{~K}\right.$, usually $\left.T_{\mathrm{G}} \sim 60 \mathrm{~K}\right)$ gas, to map out the inner $\sim 75 \mathrm{pc}$ of the $\mathrm{CMZ}$ and found high temperatures comparable to those found in previous studies.

There is a long-standing problem that the observed gas and dust temperatures do not agree throughout the CMZ (Güsten et al. 1981; Molinari et al. 2011; Ao et al. 2013; Clark et al. 2013; Ott et al. 2014). Photon-dominated region (PDR) models predict that gas and dust temperatures should match at high column densities (Hollenbach \& Tielens 1999). The observed discrepancy represents a significant problem for understanding the importance of gas temperature and pressure in the evolution of molecular clouds. The dust temperature is readily estimated via multiband continuum mapping and therefore is often used as a proxy for the dense gas temperature. While the discrepancy is not theoretically a problem, as the gas and dust may remain collisionally uncoupled up to moderately high densities given a high enough heating rate, it is an important empirical difference between $\mathrm{CMZ}$ and Galactic disk clouds. Dust and $\mathrm{NH}_{3}$ derived temperatures are usually assumed and often observed to agree in Galactic disk clouds (Pillai et al. 2006; Dunham et al. 2010; Juvela et al. 2012; Battersby et al. 2014), but the difference can no longer be ignored in the Galactic center.

Within both our own Galactic center and many nearby galactic nuclei, there is controversy over which heating mechanisms

\footnotetext{
Assuming the IAU-recommended $d=8.5 \mathrm{kpc}$ (Ghez et al. 2008; Gillessen et al. 2009; Reid et al. 2009; Gillessen et al. 2013).
}

dominate. In the CMZ, Ao et al. (2013) were able to rule out $\mathrm{X}$-ray heating as an energetically important mechanism, leaving cosmic rays and turbulence (mechanical heating) as viable candidates. Studies of the CO spectral line energy distribution (SLED) around Sgr A* and Sgr B2 argued for mechanical heating and UV heating, respectively (Goicoechea et al. 2013; Etxaluze et al. 2013). Nearby infrared-luminous galaxies have properties that resemble these regions (Kamenetzky et al. 2012, 2014), but the debate about the dominant heating mechanism remains open in many galaxies, allowing for photon-dominated, X-ray-dominated, mechanical/turbulent, or cosmic ray heating (Loenen et al. 2008; van der Werf et al. 2010; Papadopoulos et al. 2011; Meijerink et al. 2011; Bayet et al. 2011; Mangum et al. 2013; Papadopoulos \& Thi 2013).

We have expanded the $\mathrm{H}_{2} \mathrm{CO}$ mapping project of Ao et al. (2013) to a $\sim 5 \times$ larger area, including the dust ridge and the positive-longitude turbulent clouds thought to be associated with the intersection point between the $\times 1$ and $\times 2$ orbits (Rodriguez-Fernandez et al. 2006; Riquelme et al. 2013). We describe the new observations in Sect. 2. In Sect. 3, we describe the analysis process used to extract signal from the data cubes. In Sect. 4, we describe how we derive temperatures from the line ratios. We discuss various implications of our data in Sect. 5. In the appendices, we describe further details of the data reduction process, include additional tables, and provide the source code for all aspects of this project, from data reduction through figure generation for the paper.

\section{Observations and data reduction}

\subsection{Observations}

We observed the $\mathrm{CMZ}$ from $-0.4^{\circ}<\ell<1.6^{\circ}$ with the APEX-1 (SHFI) instrument (Vassilev et al. 2008) on the Atacama Pathfinder Experiment (APEX) telescope (Güsten et al. 2006) using the eXtended bandwidth Fast Fourier Transform Spectrometer (XFFTS) backend (Klein et al. 2012). The observations were performed in service mode and were spread out over two years. The time was divided into $25 \mathrm{~h}$ in June 2013, $75 \mathrm{~h}$ in April-July 2014, and $50 \mathrm{~h}$ in October 2014. A final set of observations was taken in April 2015; these data are not included in the analysis in this paper but are provided in the delivered FITS files. The time was split between the ESO (E-093.C0144A; 50h, E-095.C-0242A, 25h), MPIfR (M-091.F-0019 and M-093.F-0009; 75h), and OSO (E-093.C-0144A; 25h) queues.

The 2013 observations were taken in $4^{\prime} \times 4^{\prime}$ patches, and the frequency range covered was $217.5-220 \mathrm{GHz}$ and $216-218.5 \mathrm{GHz}$ in the two spectral windows. Scans were performed along lines of constant RA and Dec. These observations used the same observing strategy and off position as Ao et al. (2013).

The 2014 observing strategy was modified to use larger $8^{\prime} \times 8^{\prime}$ scans along lines of Galactic latitude and longitude. The frequency range was also shifted to cover windows over 217-219.5 and 218.4-220.9 GHz, thus including the bright ${ }^{13} \mathrm{CO}$ and $\mathrm{C}^{18} \mathrm{O} 2-1$ lines. Three off positions were used for these observations: (a) 17:52:06.854 -28:30:31.32; (b) 17:43:53.890 -28:07:04.68; and (c) 17:48:11.934 -29:44:41.83; position (b) exhibits some ${ }^{13} \mathrm{CO}$ emission from local clouds near $0 \mathrm{~km} \mathrm{~s}^{-1}$ but no other emission lines, while both (a) and (c) appear to be entirely clean.

Additionally, for the $\mathrm{p}-\mathrm{H}_{2} \mathrm{CO}$ data, we incorporated the $41 \mathrm{~h}$ of observations from Ao et al. (2013) using the older fast fourier transform spectrometer (FFTS) backend. These data 
covered $2 \mathrm{GHz}$ of bandwidth, including all three of the $\mathrm{p}-\mathrm{H}_{2} \mathrm{CO}$ lines and $\mathrm{SiO} 5-4$, but they did not cover the $\mathrm{CO}$ isotopologue lines.

The detected lines were $\mathrm{p}-\mathrm{H}_{2} \mathrm{CO} 3_{0,3}-2_{0,2} 218.22219 \mathrm{GHz}$, p- $\mathrm{H}_{2} \mathrm{CO} \quad 3_{2,2}-2_{2,1} \quad 218.47563 \mathrm{GHz}, \quad$ p- $\mathrm{H}_{2} \mathrm{CO} \quad 3_{2,1}-2_{2,0}$ 218.76007 GHz, SiO 5-4 217.10498 GHz, $\mathrm{CH}_{3} \mathrm{OH} 4_{2,2}-3_{1,2}$ 218.44005 GHz, OCS 18-17 $218.90336 \mathrm{GHz}, \mathrm{HC}_{3} \mathrm{~N} 24-23$ 218.32471 GHz, SO 65 $54219.94944 \mathrm{GHz}, \mathrm{HNCO} 10_{0,10}-9_{0,9}$ $219.79828 \mathrm{GHz}, \mathrm{C}^{18} \mathrm{O} 2-1219.56036 \mathrm{GHz}$, and ${ }^{13} \mathrm{CO} 2-1$ $220.39868 \mathrm{GHz}$. All listed frequencies are rest frequencies, but we do not include many, e.g., $\mathrm{CH}_{3} \mathrm{CN} 12-11$, that are in the observed band but were detected only in Sgr B2, since complete surveys of the rich molecular heimat in this band have been compiled elsewhere (Nummelin et al. 1998; Belloche et al. 2013). Of the detected lines, the SO, HNCO, $\mathrm{C}^{18} \mathrm{O}$, and ${ }^{13} \mathrm{CO}$ were not covered by the Ao et al. (2013) data but were in the newer XFFTS data. While we do not analyze these lines in this paper, we provide data cubes that include them.

The raw data were acquired with 32768 spectral channels in each window, yielding $0.1 \mathrm{~km} \mathrm{~s}^{-1}$ resolution. We expect to see no lines narrower than a few $\mathrm{km} \mathrm{s}^{-1}$ in the $\mathrm{CMZ}$, particularly not with the relatively shallow observations we have acquired. We therefore downsampled the data by a factor of 8 to $0.8 \mathrm{~km} \mathrm{~s}^{-1}$ resolution prior to resampling onto a $1 \mathrm{~km} \mathrm{~s}^{-1}$ grid to make the data more manageable.

The system temperature ranged from $120<T_{\text {sys }}<200 \mathrm{~K}$ for the majority of the observations, with a mean $T_{\mathrm{sys}}=165 \mathrm{~K}$. A small fraction $(10 \%)$ of observations were in the range $200<$ $T_{\text {sys }}<300 \mathrm{~K}$. There were also a very small number $(<1 \%)$ with much higher system temperatures $\left(300<T_{\text {sys }}<750 \mathrm{~K}\right)$ that were flagged out (see Sect. 2.2.2).

\subsection{Reduction}

\subsubsection{Calibration}

Calibration was performed at the telescope using the standard APEX calibration tools ${ }^{2}$. These yield flux-calibrated spectra at each position with appropriate pointing information. Typical flux calibration uncertainties are $\sim 10 \%$ and pointing errors $\sigma \lesssim 2^{\prime \prime}$.

There was a significant calibration error discovered at the APEX telescope during a large segment of the 2014 observing campaign ${ }^{3}$. The calibration errors were of order $\sim 15-25 \%$. We corrected the data using the correction factors suggested on the calibration page, but the remaining calibration uncertainty is higher in this data, $\sim 15 \%$ total rather than the usual $\sim 10 \%$.

\subsubsection{Flagging bad spectra}

Spectra were removed if they showed excessive noise compared to the theoretical expectation given the measured system temperature. As in Ao et al. (2013), the threshold was set to $1.5 \times$ the theoretical noise from averaging two polarizations, i.e. $3 T_{\text {sys }}\left(2 \Delta v t_{\text {exp }}\right)^{-0.5}$, where $t_{\text {exp }}$ is the exposure time (integration time) per spectrum in seconds and $\Delta v$ is the channel width in Hz. This approach resulted in $\sim 0.2 \%$ of the data being removed.

\footnotetext{
2 See the observing manuals:

http: //www . apex-telescope.org/documents/public/ APEX-MPI-MAN-Q012.pdf; http://wWW . apex-telescope. org/documents/public/APEX-MPI-MAN-0011.pdf

3 http://wWW . apex-telescope.org/heterodyne/shfi/ calibration/calfactor/
}

At the position of Sgr B2, the noise was significantly higher due to signal from the continuum source. We therefore disabled this flagging in a $2.5^{\prime}$ box around Sgr B2.

\subsubsection{Mapmaking}

The maps were made by computing an output grid in Galactic coordinates with $7.2^{\prime \prime}$ pixels and adding each spectrum to the cube at the appropriate pixel $^{4}$ location. In order to avoid empty pixels and maximize the signal-to-noise, the spectra were added to the grid with a weight set from a Gaussian with $F W H M=10^{\prime \prime}$, which effectively smooths the output images from $F W H M \approx 28^{\prime \prime}$ to $\approx 30^{\prime \prime}$. See Mangum et al. (2007) for more detail on the on-the-fly mapping technique used here. The spectra were averaged with inverse-variance weighting. The full weight equation is therefore:

$W_{i}=\exp \left(\frac{-\left(\left(x-x_{i}\right)^{2}+\left(y-y_{i}\right)^{2}\right)}{2 \sigma_{\text {pix }}^{2}}\right) \frac{1}{\sigma_{\text {rms }}^{2}}$

where $x_{i}$ and $y_{i}$ are the coordinate of the pointing in pixel space, $\sigma_{\text {pix }} \approx 10^{\prime \prime} / \sqrt{8 \log 2} /\left(7.2^{\prime \prime}\right.$ pix $\left.^{-1}\right)=1.38$, and $\sigma_{\text {rms }}$ is the standard deviation measured along the spectrum in a signal-free frequency region.

The position-position-velocity (PPV) cubes were created with units of brightness temperature on the corrected antenna temperature scale $\left(T_{\mathrm{A}}^{*}\right)$. The main beam efficiency is $\eta_{\mathrm{mb}}=0.75$ $(\text { gain } \sim 39 \mathrm{Jy} / \mathrm{K})^{5}$. These values are noted in the FITS headers of the released data.

The maps achieved a depth of $\sigma=50-80 \mathrm{mK}$ in $1 \mathrm{~km} \mathrm{~s}^{-1}$ channels and $F W H M=30^{\prime \prime}$ beams. The noise is slightly lower (about 15\%) than expected from the online APEX calculator because our noise measurements are made in moderately smoothed maps.

\subsection{Baselining}

The data showed significant baseline structure, leading to largescale correlated components in the resulting spectra. The baselines were removed over a velocity range $-150<v_{\mathrm{LSR}}<$ $150 \mathrm{~km} \mathrm{~s}^{-1}$ by the following procedure:

1. identify bright PPV regions in the $\mathrm{p}-\mathrm{H}_{2} \mathrm{CO} 3_{0,3}-2_{0,2}$ line using the technique described in Sect. 3;

2. mask the regions identified as $3_{0,3}-2_{0,2}$-bright for each other spectral line data cube;

3. fit a polynomial to the un-masked data;

4. subtract the fitted polynomial.

We used a 7th-order polynomial for this process because lower order baseline removal left a significant and patterned residual. A more detailed examination of the baseline removal process is described in Appendix A.

\section{Signal extraction and masking}

We use the method described partially in Ao et al. (2013) and more thoroughly in Dame (2011) to mask the data cubes at locations of significant signal in the brightest line. A noise map

\footnotetext{
4 We use the term "pixel" to refer to a square data element projected on the sky with axes in Galactic coordinates. The term "voxel" is used to indicate a cubic data element, with two axes in galactic coordinates and a third in frequency or velocity.

5 http://www . apex-telescope.org/telescope/efficiency/
} 
$20 \mathrm{kms}$

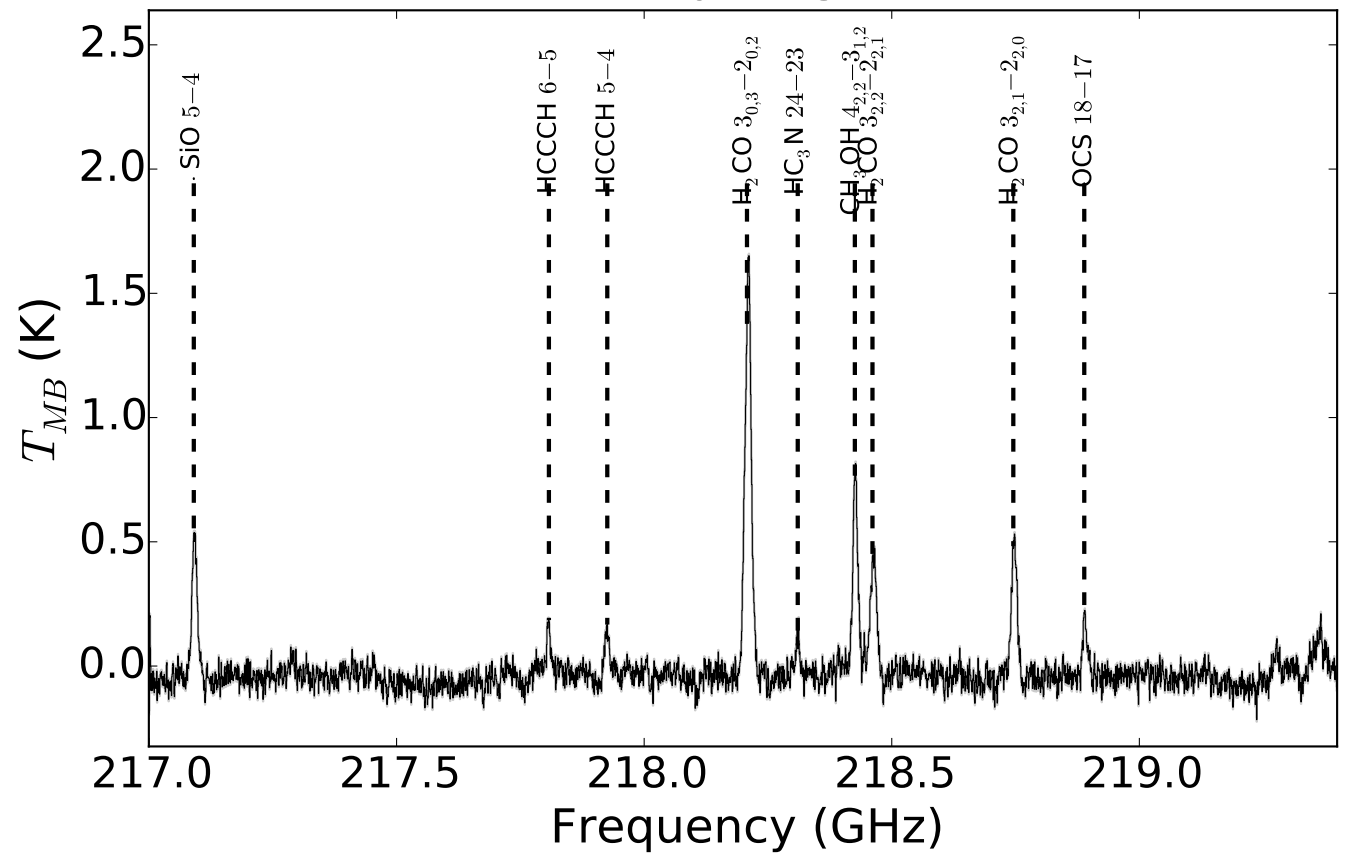

$20 \mathrm{kms}$

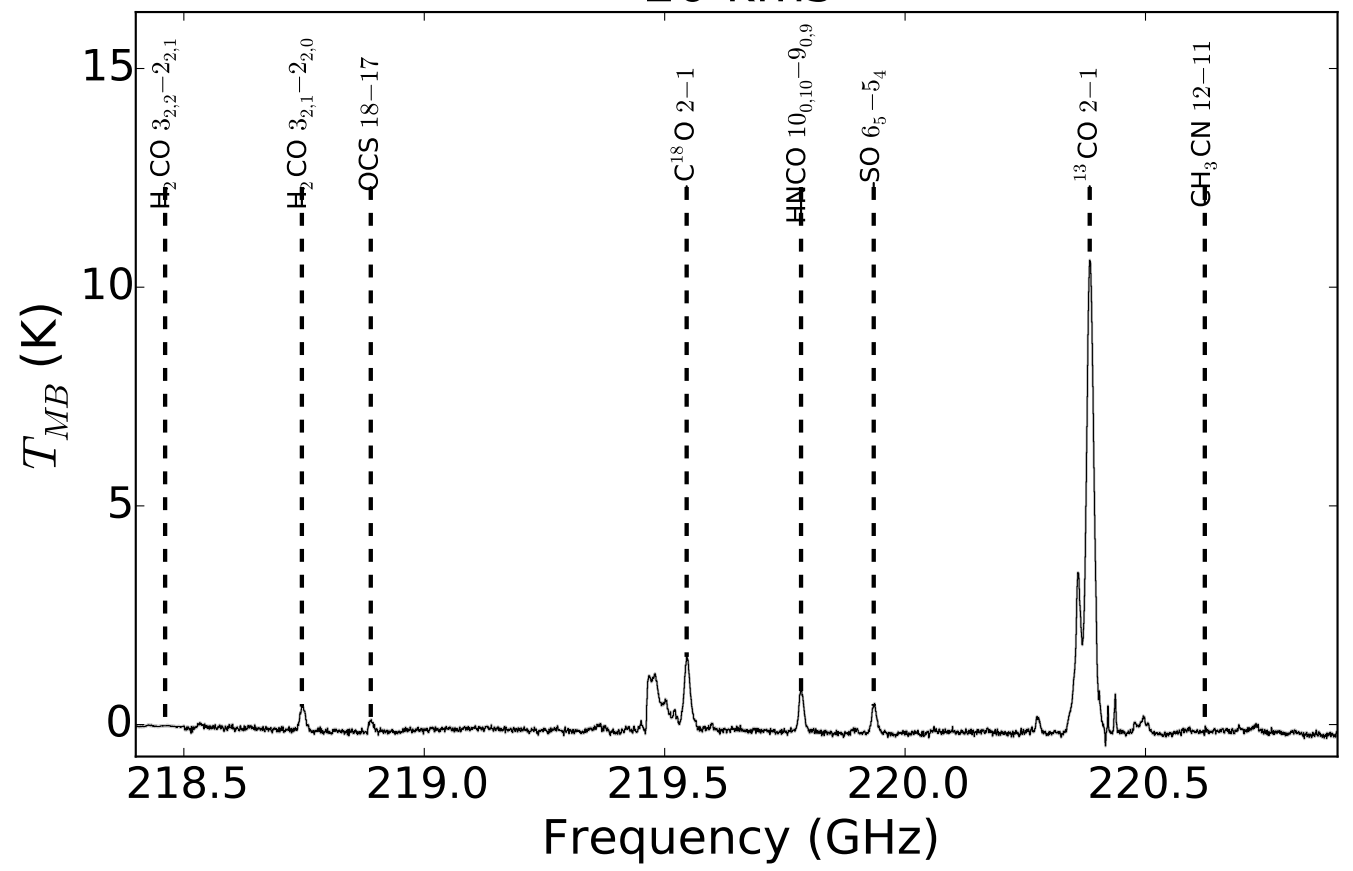

Fig. 1. Spectra toward one $\mathrm{H}_{2}$ CO-bright cloud, the $20 \mathrm{~km} \mathrm{~s}^{-1}$ cloud $(\ell=359.892, b=-0.074)$, over the full APEX band. All detected lines are identified. The feature overlapping the $\mathrm{C}^{18} \mathrm{O} 2-1$ line at about $219.5 \mathrm{GHz}$ is the ${ }^{12} \mathrm{CO}$ line from the upper sideband, suppressed by $\sim 20 \mathrm{~dB}$. There are artifacts at the band edges, e.g., below $217.0 \mathrm{GHz}$, because these spectra average a few different tunings without accounting for missing data at the band edges.

was created by computing the sample standard deviation over a $200 \mathrm{~km} \mathrm{~s}^{-1}$ range in which no signal was present. We use the $\mathrm{p}-\mathrm{H}_{2} \mathrm{CO} 3_{0,3}-2_{0,2}$ line, which is the brightest of the $\mathrm{H}_{2} \mathrm{CO}$ lines, to create the mask by the following procedure:

1. Smooth the data with a Gaussian of width two pixels in each direction (spatial, $\sigma=14.4^{\prime \prime}$, and spectral, $\sigma=2 \mathrm{~km} \mathrm{~s}^{-1}$ ).

2. Create an inclusive mask of all pixels with brightness $T_{\mathrm{A}}>$ $2 \sigma$ in the smoothed map.
3. Grow the mask from the previous step by one pixel in each direction (this is known in image processing as binary dilation).

The $\mathrm{p}-\mathrm{H}_{2} \mathrm{CO} 3_{0,3}-2_{0,2}$ mask was then applied to the $3_{2,1}-2_{2,0}$ and $3_{2,2}-2_{2,1}$ cubes created with the same PPV gridding. There is some overlap between the $\mathrm{CH}_{3} \mathrm{OH} 4_{2,2}-3_{1,2}$ line and the $\mathrm{p}-\mathrm{H}_{2} \mathrm{CO}$ $3_{2,2}-2_{2,1}$ line in PPV space, so we shifted the $\mathrm{p}-\mathrm{H}_{2} \mathrm{CO} 3_{0,3}-2_{0,2}$ mask to the velocity of the $\mathrm{CH}_{3} \mathrm{OH}$ line in the $\mathrm{p}-\mathrm{H}_{2} \mathrm{CO} 3_{2,2}-2_{2,1}$ cube and excluded those pixels expected to contain $\mathrm{CH}_{3} \mathrm{OH}$ 
emission. However, inspection of the $3_{2,2}-2_{2,1}$ cube revealed that significant chunks of the $3_{2,2}-2_{2,1}$ signal were excised by this masking, so all further analysis excludes the $3_{2,2}-2_{2,1}$ line.

Integrated intensity maps are shown in Fig. 2a for the $3_{0,3}-2_{0,2}$ map masked with the above method and in Fig. $2 b$ for the $3_{2,1}-2_{2,0}$ map using the mask created from the $3_{0,3}-2_{0,2}$ data.

\subsection{Ratio maps}

We have created $\mathrm{p}-\mathrm{H}_{2} \mathrm{CO} S_{v}\left(3_{2,1}-2_{2,0}\right) / S_{v}\left(3_{0,3}-2_{0,2}\right)$ ratio maps as a first step toward building temperature maps. In general, a higher $\mathrm{p}-\mathrm{H}_{2} \mathrm{CO} S_{v}\left(3_{2,1}-2_{2,0}\right) / S_{v}\left(3_{0,3}-2_{0,2}\right)$ indicates a higher temperature, and therefore the ratio maps are a good proxy for relative temperature. We display four different varieties of ratio map in Figs. 3 and E.1.

Figure 3 shows maps produced by creating ratio cubes, in which each voxel has the value $R_{1}=\mathrm{p}-\mathrm{H}_{2} \operatorname{COS}_{v}\left(3_{2,1}-2_{2,0}\right) /$ $S_{v}\left(3_{0,3}-2_{0,2}\right)$, then averaging across velocity with constant weight for each voxel in Fig. $3 \mathrm{a}$ and with the weight set by the $3_{0,3}-2_{0,2}$ brightness value in Fig. $3 b$. In both cases, the mask described in Sect. 3 was applied to the cubes before averaging over velocity.

Ratio maps are subject to dramatic uncertainties in the low signal-to-noise regime, which is inevitably reached in some parts of the map. The error distribution on the ratio becomes nonGaussian as signal approaches zero, approaching a pathological Cauchy distribution in which the mean becomes undefined. We therefore limited our ratio measurements to regions with significance $>5 \sigma$ in the $3_{0,3}-2_{0,2}$ line and used that line as the denominator in the ratio. By using the brighter line as the denominator, we avoid divide-by-zero numerical errors.

\subsection{Region extraction}

In order to extract higher signal-to-noise measurements on selected regions, we broke the data down into subsets using both a by-eye region selection drawing regions using ds 9 and a more systematic approach using a dendrogram-based clump finding algorithm (Rosolowsky et al. 2008) ${ }^{6}$. The dendrogram clump finding uses a watershed decomposition like other clump finding methods, but it also extracts clumps at different brightness scales (different heights in the watershed) and tracks how nested clumps are related to each other. Within a hierarchy of nested clumps, the brightest and smallest is a "leaf" and all associated larger structures are "ancestors" (roots or trunks and branches). We refer to the clumps identified in this manner as "dendrogramextracted" clumps from here on. Figures 1 and 3 in Rosolowsky et al. (2008) give visual representations of this hierarchy.

We ran the dendrogram extraction analysis on the $3_{0,3}-2_{0,2}$ PPV cube data. We assumed a uniform noise per pixel $\sigma_{\mathrm{T}}=$ $\left\langle\sigma_{\mathrm{T}}\right\rangle=0.07 \mathrm{~K}$, which corresponds to the mean noise per field. We enforced thresholds of a minimum number of voxels $N_{\text {min }}=100$, a peak threshold of $I=3 \sigma$, and a splitting threshold $\Delta=2 \sigma$, which defines how large the brightness difference between the peak pixel and an adjacent pixel must be in order to divide the clump into two sources. The exact values of these parameters are not particularly important, as we are interested in general trends with size-scale and galactocentric distance, but we caution against overinterpretation of the resulting catalog as the number of sources and their size and distribution can change dramatically with small changes in the selected parameters. The

\footnotetext{
6 http://dendrograms.org/
}

number and location of voxels included in the catalog is, however, relatively robust against small parameter changes.

For each extracted clump, we measured the corresponding integrated and peak emission in the $3_{0,3}-2_{0,2}, 3_{2,1}-2_{2,0}$, and ${ }^{13} \mathrm{CO}$ cubes. We also extracted the mean dust temperature and column density from SED fits to Herschel HiGal 170-500 $\mu \mathrm{m}$ maps (Molinari et al. 2010; Traficante et al. 2011). The SED fits were performed on background-subtracted data using an approach originally described in Battersby et al. (2011), which includes details of the fitting process and specification of assumed physical parameters. We also extracted dust column density and temperature using a more naive pixel-by-pixel approach with no background subtraction ${ }^{7}$ and found the two to be consistent. We assumed a dust to gas ratio of 100 and dust opacity $\kappa_{505 \mathrm{GHz}}=4 \mathrm{~g} \mathrm{~cm}^{-2}$, the same as Battersby et al. (2011) and equivalent to that assumed in the Bolocam Galactic Plane Survey observations of the CMZ with $\beta=2\left(\kappa_{271.1 \mathrm{GHz}}=1.1 \mathrm{~g} \mathrm{~cm}^{-2}\right.$ Bally et al. 2010; Aguirre et al. 2011; Ginsburg et al. 2013).

\subsubsection{Manual region extraction}

The by-eye selected regions were extracted less systematically, instead focusing on peaks in the $3_{0,3}-2_{0,2}$ or $3_{2,1}-2_{2,0}$ emission. These selections were made in two dimension rather than in the full PPV space. We included a series of large square $8^{\prime} \times 8^{\prime}$ apertures corresponding to each observed field. For each region extracted in this fashion, we fit a 1-3 component model as described in Sect. 4.2, where each component consists of a 6-parameter multi-Gaussian model. An example spectrum extracted from a small region around "cloud c" is shown in Fig. 5. The extracted regions are shown in Fig. B.1.

\section{Analysis: temperature determination}

In this section, we describe the techniques used to derive gas temperatures. In Sect. 4.1 we model the line ratios with a radiative transfer code. We fit Gaussians to spectra extracted from the cubes and use these fits to measure line ratios in Sect. 4.2. In Sect. 4.3, we apply the same modeling approach to the dendrogram-extracted clumps from Sect. 3.2. We use the modeling results from Sects. 4.2 and 4.3 to justify a simplified modeling approach that we apply to the entire map of the CMZ in Sect. 4.4.

\subsection{Radiative transfer modeling}

We use RADEX (van der Tak et al. 2007) and a related solver (Fujun Du's myRadex ${ }^{8}$ ) to create model grids for the $\mathrm{p}-\mathrm{H}_{2} \mathrm{CO}$ lines over a grid of 20 densities $\left(n=10^{2.5}-10^{7} \mathrm{~cm}^{-3}\right)$, a fixed assumed ${ }^{9}$ line gradient of $5 \mathrm{~km} \mathrm{~s}^{-1} \mathrm{pc}^{-1}, 30 \mathrm{H}_{2} \mathrm{CO}$ column densities $N\left(\mathrm{H}_{2} \mathrm{CO}\right)=1 \times 10^{11}-1 \times 10^{15.1} \mathrm{~cm}^{-2}$, and 50 temperatures from 10 to $350 \mathrm{~K}$. The grid was created using the pyradex wrapper of RADEX ${ }^{10}$ and collision rates retrieved from LAMDA (Wiesenfeld \& Faure 2013). We then upsampled these grids by spline interpolating between grid points to acquire a high-resolution $\left(T_{\mathrm{G}} \times n \times N=250 \times 100 \times 101\right)$

\footnotetext{
7 http://hi-gal-sed-fitter.readthedocs.org/en/latest/ 8 https://github.com/fjdu/myRadex

9 The line gradient is selected because observed line widths have FWHM larger than $5 \mathrm{~km} \mathrm{~s}^{-1}$ in $30^{\prime \prime}(1.2 \mathrm{pc})$ beams throughout the CMZ. The temperature measurements are robust against this assumption, with a typical change of $\mathrm{d} T / T<25 \%$ for a gradient $4 \times$ larger or smaller.

${ }^{10}$ https://github.com/adamginsburg/pyradex
} 


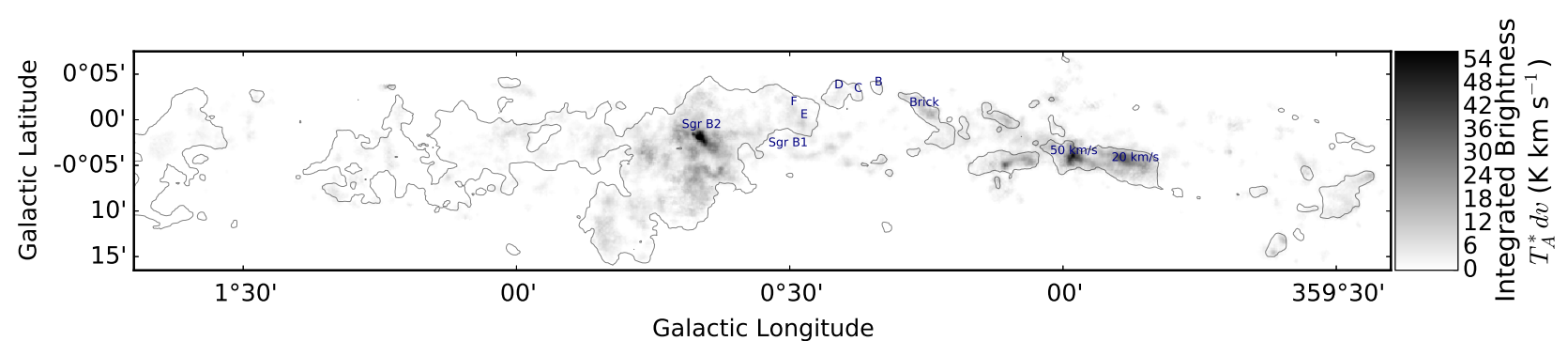

(a)

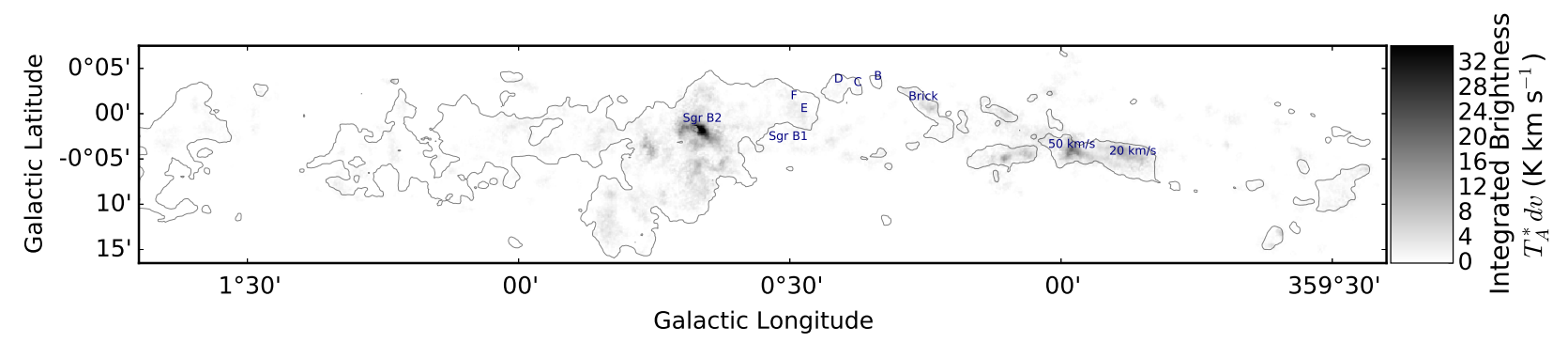

(b)

Fig. 2. Integrated intensity (moment 0 ) maps of the a) $\mathrm{p}-\mathrm{H}_{2} \mathrm{CO} 3_{0,3}-2_{0,2}$ and b) $3_{2,1}-2_{2,0}$ lines. The cubes were masked prior to integration using the method described in Sect. 3. Selected region names are labeled. The thin black contours are at a column $N\left(\mathrm{H}_{2}\right)=5 \times 10^{22} \mathrm{~cm}^{-2}$ from the Herschel SED fit maps.

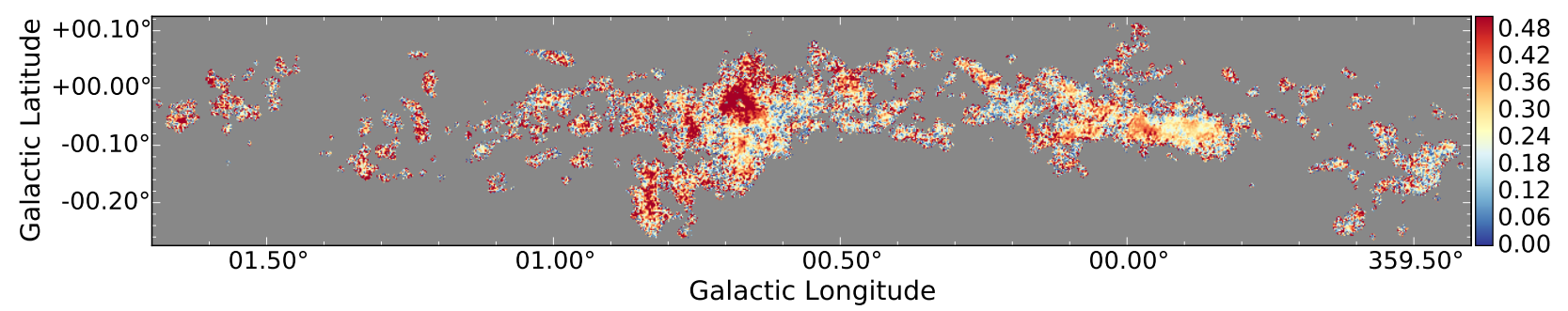

(a)

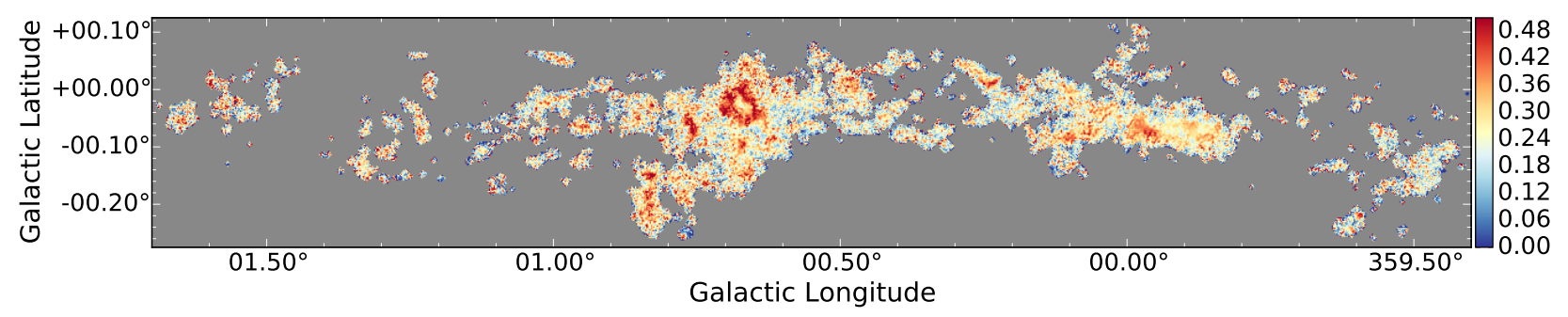

(b)

Fig. 3. a) Map of the ratio of the integrated emission of $\mathrm{p}-\mathrm{H}_{2} \mathrm{CO} S_{v}\left(3_{2,1}-2_{2,0}\right) / S_{v}\left(3_{0,3}-2_{0,2}\right)$. The gray areas indicate pixels that were completely masked out in the PPV cubes. The Sgr B2 peaks exhibit $\mathrm{H}_{2} \mathrm{CO}$ absorption and therefore do not contain reliable measurements. Higher ratios (red) correspond to higher temperatures. b) The same as a), but the average along velocity has been weighted by the $3_{0,3}-2_{0,2}$ brightness.

grid covering $10<T_{\mathrm{G}}<350 \mathrm{~K}, 10^{2.5}<n<10^{7} \mathrm{~cm}^{-3}$, and $10^{11}<N<10^{15.1} \mathrm{~cm}^{-2} \mathrm{~km} \mathrm{~s}^{-1} \mathrm{pc}^{-1}$. For our analysis, we used the myRadex grid; it is consistent with the RADEX grid over most of parameter space but suffers fewer numerical instabilities over the region we are interested in.

\subsection{Spectral modeling}

We extracted spectra averaged over selected regions and fitted a 6-parameter model to the full $218-219 \mathrm{GHz}$ spectral range using pyspeckit (Ginsburg \& Mirocha 2011). The fitted parameters are the amplitude of the $\mathrm{H}_{2} \mathrm{CO} 3_{0,3}-2_{0,2}$ line, the centroid velocity ${ }^{11}$, the line width $(\sigma)$, the ratio $R_{1}=\mathrm{p}-\mathrm{H}_{2} \mathrm{CO} S_{v}\left(3_{2,1}-2_{2,0}\right) / S_{v}\left(3_{0,3}-2_{0,2}\right)$, the ratio $R_{2}=$ $\mathrm{p}-\mathrm{H}_{2} \mathrm{CO} S_{v}\left(3_{2,2}-2_{2,1}\right) / S_{v}\left(3_{2,1}-2_{2,0}\right)$, and the amplitude of the $\mathrm{CH}_{3} \mathrm{OH} 4_{2,2}-3_{1,2}$ line ${ }^{12}$, where $S_{v}$ is the surface brightness. For these spectra, we performed a different baseline subtraction to

11 We assume the $\mathrm{CH}_{3} \mathrm{OH}$ and $\mathrm{H}_{2} \mathrm{CO}$ lines have the same centroid velocity, and we find no counterexamples to that assumption within our data.

12 The parameters $R_{2}$ and the $\mathrm{CH}_{3} \mathrm{OH}$ brightness are essential for fully fitting the spectrum, but they are not used for further analysis. 


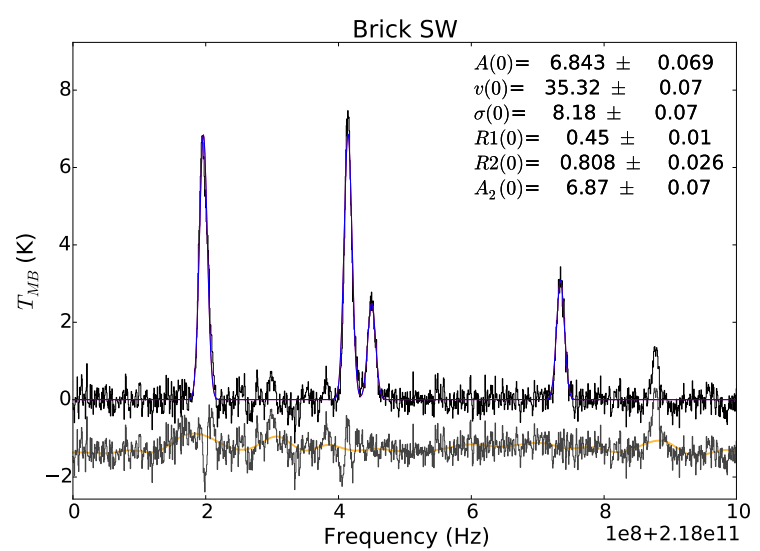

(a)

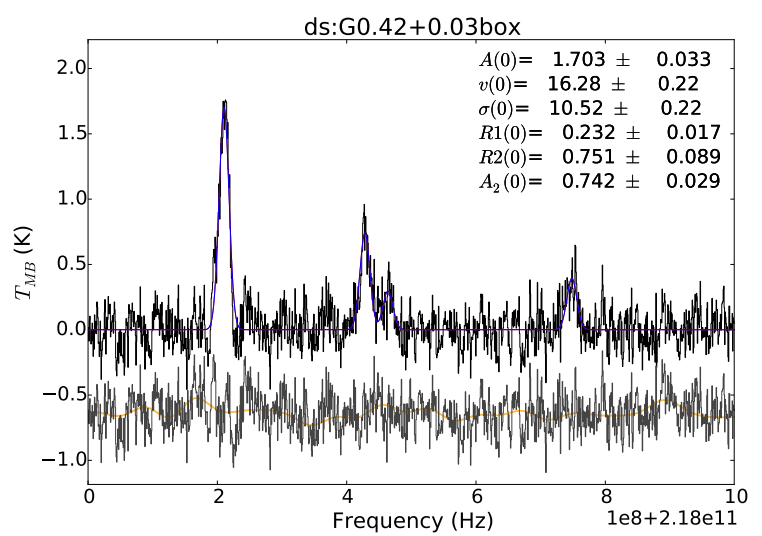

(b)

Fig. 4. Simultaneous fits to four lines, from low to high frequency, $\mathrm{p}-\mathrm{H}_{2} \mathrm{CO} 3_{0,3}-2_{0,2}, \mathrm{CH}_{3} \mathrm{OH} 4_{2,2}-3_{1,2}, \mathrm{p}-\mathrm{H}_{2} \mathrm{CO} 3_{2,2}-2_{2,1}$, and p- $\mathrm{H}_{2} \mathrm{CO} 3_{2,1}-2_{2,0}$ for a) The Brick $\left(\ell=0.241, b=0.006, r=25^{\prime \prime}\right)$ and b) a box centered on G0.42+0.03 $\left(\ell=0.415, b=0.032, \approx 80^{\prime \prime} \times 80^{\prime \prime}\right)$. The fitted parameters shown in the legend are the amplitude of the $\mathrm{H}_{2} \mathrm{CO} 3_{0,3}-2_{0,2}$ line, the centroid velocity, the line width $(\sigma$, not FWHM), the ratio $R_{1}=\mathrm{p}-\mathrm{H}_{2} \mathrm{CO} S_{v}\left(3_{2,1}-2_{2,0}\right) / S_{v}\left(3_{0,3}-2_{0,2}\right)$, the ratio $R_{2}=\mathrm{p}-\mathrm{H}_{2} \operatorname{CO} S_{v}\left(3_{2,2}-2_{2,1}\right) / S_{v}\left(3_{2,1}-2_{2,0}\right)$, and the amplitude of the $\mathrm{CH}_{3} \mathrm{OH} 4_{2,2}-3_{1,2}$ line. These spectra have significantly different ratios and therefore derived temperatures: The Brick has $T_{\mathrm{G}} \approx 100 \mathrm{~K}$ while G0.42+0.03 has $T_{\mathrm{G}} \approx 40-50 \mathrm{~K}$. The bottom black spectrum is the residual, and the orange wiggly spectrum shows the spline fit used to remove baseline ripples. The OCS 18-17 $218.90336 \mathrm{GHz}$ and $\mathrm{HC}_{3} \mathrm{~N} 24-23218.32471 \mathrm{GHz}$ lines are also detected, but not fitted, in The Brick. In these examples, we are showing cases where the $\mathrm{CH}_{3} \mathrm{OH} 4_{2,2}-3_{1,2}$ and p- $\mathrm{H}_{2} \mathrm{CO} 3_{2,2}-2_{2,1}$ lines are not too badly blended, but along many lines of sight, especially those within $\sim 20^{\prime}$ of Sgr B2, they are irretrievably blended.

that described in Sect. 2.3. We first fit the described 6-parameter model, then fit a spline curve to the residual smoothed to a 50-channel $\left(50 \mathrm{~km} \mathrm{~s}^{-1}\right)$ scale. We then re-fit the model to the data. In all but the lowest signal-to-noise cases, this approach resulted in consistent ratio measurements between the two fits and with the other methods. Figure 4 shows two example spectra that exhibit significantly different ratios with the spline baseline fit indicated. We use the baseline-subtracted measurements for further analysis.

For each extracted region (see Sect. 3.2), we created $\chi^{2}$ grids over the modeled temperature-density-column density parameter space. We measured $\chi^{2}$ with five independent constraints: the line ratio $R_{1}$, the $\mathrm{H}_{2} \mathrm{CO}$ abundance, the total column density of $\mathrm{H}_{2}$, a lower limit on the $\mathrm{H}_{2}$ volume density, and an upper limit on the beam filling factor ${ }^{13}$. We use the line ratio as the primary constraint rather than line brightnesses to avoid uncertainties due to the filling factor of the emitting gas: even for diffuse clouds, the filling factor of the emitting regions may be ff $\ll 1$ if the emission is isolated to compact shocked regions, as expected if highly supersonic $\mathcal{M}>10$ turbulence is energetically dominant in the clouds. We use an abundance $N\left(\mathrm{p}-\mathrm{H}_{2} \mathrm{CO}\right) / N\left(\mathrm{H}_{2}\right)=X\left(\mathrm{p}-\mathrm{H}_{2} \mathrm{CO}\right)=10^{-9.08 \pm 1}$, allowing for more than two orders of magnitude variation in the $\mathrm{H}_{2} \mathrm{CO}$ abundance (Ginsburg et al. 2013; Carey et al. 1998; Wootten et al. 1978; Mundy et al. 1987). To constrain the total column density, we use the Herschel dust maps (see Sect. 3.2) to derive an $\mathrm{H}_{2}$ column density, which has a nominal $\sim 2-3 \times$ uncertainty ${ }^{14}$. We treat the error as $10 \times$ (instead of $\sim 2-3 \times$ ) to account for the additional uncertainty in the abundance of $\mathrm{p}-\mathrm{H}_{2} \mathrm{CO}$. The effective range of $N\left(\mathrm{p}-\mathrm{H}_{2} \mathrm{CO}\right)$ is therefore $10^{11.5}<N<10^{15.5} \mathrm{~cm}^{-2}$,

\footnotetext{
${ }_{13}$ Where the model predicts a brightness temperature higher than observed, we can invoke a low filling factor to explain the discrepancy. If the model predicts a brightness temperature too low, we can rule it out. 14 The statistical uncertainty is much smaller than the systematic unknowns, including the dust opacity per unit mass, the presence of multiple line-of-sight cloud components, and the presence of multiple temperature components within any given cloud.
}

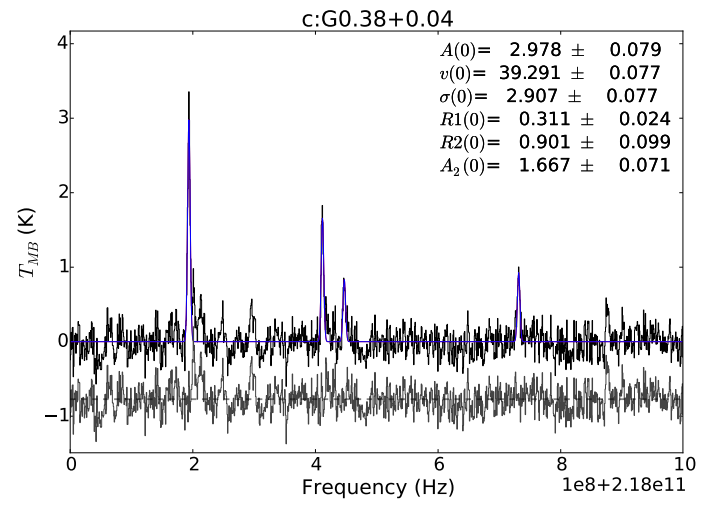

Fig. 5. Fitted spectrum of "cloud c". The fitted parameters and their corresponding errors are shown in the legend as in Fig. 4.

allowing for $10^{21.5}<N\left(\mathrm{H}_{2}\right)<10^{23.5} \mathrm{~cm}^{-2}$ and $\pm 1 \sigma$ in log abundance. To constrain the dust density, we assume the selected area has a mass given by the Herschel dust-derived mass and a spherical volume, which sets a conservative (but nonetheless highly constraining) lower limit on the volume density.

These constraints are shown projected onto the three planes of our fitted parameter space in the multi-paneled Figs. D.1-D.4 for one example region. The fitted parameters are displayed in Fig. D.4.

While $R_{2}$ can provide significant constraints on various parameters, we do not use it for our general analysis because of the ambiguity imposed by the overlap with the $\mathrm{CH}_{3} \mathrm{OH}$ $4_{2,2}-3_{1,2}$ line. Blending between the $\mathrm{CH}_{3} \mathrm{OH} 4_{2,2}-3_{1,2}$ and $\mathrm{H}_{2} \mathrm{CO} 3_{2,2}-2_{2,1}$ line occurs frequently, in $\sim 1 / 4$ of all sightlines.

\subsection{Clump modeling}

We derived temperatures for each dendrogram catalog clump using the same measured quantities as in Sect. 4.2 as constraints. 


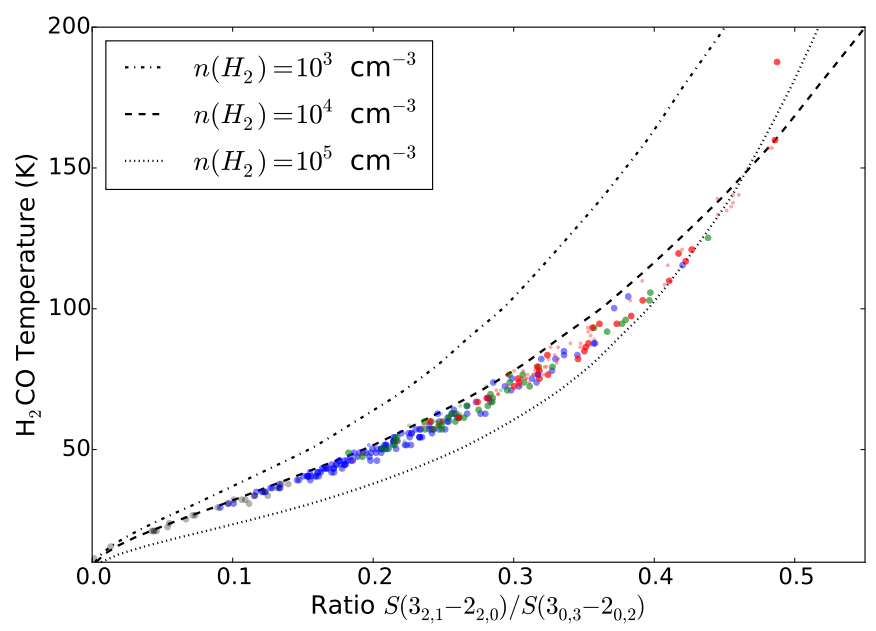

Fig. 6. Derived temperature vs. the measured ratio p- $\mathrm{H}_{2} \mathrm{CO} S_{v}\left(3_{2,1}-2_{2,0}\right) / S_{v}\left(3_{0,3}-2_{0,2}\right)$ for the dendrogram clumps. The big symbols represent leaves (compact clumps) and the small symbols represent larger and therefore lower-density ancestor structures. The temperature includes constraints from the assumed constant $\mathrm{H}_{2} \mathrm{CO}$ abundance and the varying column density. The points are color coded by signal-to-noise in the ratio $R_{1}$, with gray $S / N<5$, blue $5<S / N<25$, green $25<S / N<50$, and red $S / N>50$. The black lines show the modeled temperature as a function of $R_{1}$ for three different assumed densities with a constant assumed abundance $X_{\mathrm{H}_{2} \mathrm{CO}}=1.2 \times 10^{-9}$. While the measured values appear consistent with the $n=10^{4} \mathrm{~cm}^{-3}$ curve, the systematic errors permit any density in the range $10^{4} \mathrm{~cm}^{-3}<n<10^{5} \mathrm{~cm}^{-3}$; this plot most importantly shows that $n \sim 10^{3} \mathrm{~cm}^{-3}$ is not plausible and that the temperature does not differ very much for a given value of $R_{1}$ over the plausible range of densities.

The $\chi^{2}$ grid described in Sect. 4.1 was used to extract the temperature and associated error.

We plot the derived temperature for all clumps against the measured line ratio in Fig. 6. This figure includes both "leaves" (the most compact of the nested clumps) and "ancestors" (any clump that contains a smaller clump within it) from the dendrogram extraction. The figure illustrates the effects of assuming higher or lower density on the temperature; the derived temperature is only weakly dependent on the assumed density. The lower density models are excluded by the observed volume density lower limit (assuming spherical geometry) from the dust. We use an assumed density $n\left(\mathrm{H}_{2}\right)=10^{4} \mathrm{~cm}^{-3}$ when a uniform assumed density is required in further analysis.

\subsection{Temperature maps}

Using the relations shown in Fig. 6, we converted the ratio map shown in Fig. 3 to a temperature map in Fig. 7 with a constant abundance $X\left(\mathrm{H}_{2} \mathrm{CO}\right)=1.2 \times 10^{-9}$ and two constant densities $n=10^{4}$ (Figs. 7a/b) and $n=10^{5} \mathrm{~cm}^{-3}$ (Fig. 7c). Because this map includes the full line-of-sight integrated emission, there are many regions where multiple independent components are being mixed.

These temperature maps do not have uniform reliability. There is some spatial variation in the noise, but the variation in the signal is much more important. In regions where the peak signal is low, the reliability of these maps is substantially reduced. We have also applied a mask to the lower panels of Fig. 7 to make the low-reliability regions blend into the background by adding a foreground gray layer with opacity set by the inverse $\mathrm{S} / \mathrm{N}$ (see Appendix E).
The effective range of detected temperatures is $\approx 40-150 \mathrm{~K}$. While lower and higher temperatures appear in the map, those measurements should not be trusted. Below $T_{\mathrm{G}} \lesssim 40 \mathrm{~K}$, all temperature measurements in the map are effectively $1 \sigma$ upper limits $^{15}$. This limit is due to a combination of sensitivity and excitation: below $40 \mathrm{~K}$, the $\mathrm{p}-\mathrm{H}_{2} \mathrm{CO}$ lines are observed to be faint; the $3_{2,1}-2_{2,0}$ line is not detected. In regions of high column density, we detect both lines, and in all of these regions, we have measured high temperatures $\left(T_{\mathrm{G}}>40 \mathrm{~K}\right)$. Since $T_{\mathrm{G}} \sim 40 \mathrm{~K}$ corresponds to $R_{1} \sim 0.15$, a $5 \sigma$ temperature measurement would require $T_{\mathrm{B}}\left(3_{2,1}-2_{2,0}\right)=(5 \times 0.07 \mathrm{~K})=0.35 \mathrm{~K}$ and $T_{\mathrm{B}}\left(3_{0,3}-2_{0,2}\right)=$ $(5 \times 0.07 \mathrm{~K}) / 0.15=2.33 \mathrm{~K}$. This $T_{\mathrm{B}}\left(3_{0,3}-2_{0,2}\right)$ is slightly brighter than the brightest detection in our map, and all regions detected with such high brightness temperatures also turned out to have higher $T_{\mathrm{B}}\left(3_{2,1}-2_{2,0}\right)$, and therefore higher $R_{1}$ and temperature. The higher measured temperatures become lower limits above $\sim 150 \mathrm{~K}$ because the $\mathrm{p}-\mathrm{H}_{2} \mathrm{CO}$ line ratio is intrinsically insensitive to higher temperatures, as can be seen in Fig. D.4 (rightmost column), in which the modeled line brightnesses are both nearly constant above $T_{\mathrm{G}} \gtrsim 150 \mathrm{~K}$.

\section{Discussion}

\subsection{Gas heating and thermal balance}

Following Ao et al. (2013) and Papadopoulos (2010), we examine the energy balance in the CMZ gas to understand the temperatures we measure. The two key processes they identified are cosmic ray and turbulent dissipation (shock) heating. We ignore all UV-driven heating processes in this section as we are interested in gas at high extinction $\left(A_{V}>10\right)^{16}$. Other processes, e.g., direct (proto)stellar heating or supernova bubble interaction may be relevant locally, but cannot heat the gas globally. Ao et al. (2013) ruled out diffuse X-rays as a significant heating source, with an energy density $\sim 3$ orders of magnitude too low to explain observed temperatures.

We determine the expected gas kinetic temperature by assuming that the heating and cooling processes are in equilibrium. This is generally a good assumption on the scales we observe, as typical gas cooling rates via line or dust cooling around $T_{\mathrm{G}} \sim 100 \mathrm{~K}$ and $n \sim 10^{5} \mathrm{~cm}^{-3}$ are $\mathrm{d} T / \mathrm{d} t \sim$ $10^{-20} \mathrm{erg} \mathrm{s}^{-1} \mathrm{~cm}^{-3} \sim 23\left(n / 10^{5} \mathrm{~cm}^{-3}\right) \mathrm{K} \mathrm{kyr}^{-1}$ (Goldsmith 2001, and see below). The cooling timescale is shorter than the timescales for any energy injection mechanisms that may plausibly heat the gas. The supernova rate is $<1 \mathrm{kyr}^{-1}$ over the whole CMZ, and supernova remnants stop producing cosmic rays rapidly, so the supernovae are expected to contribute to a diffuse cosmic ray population rather than local stochastic heating incidents. Turbulence in these clouds is dynamically driven on scales of 10-30 pc (Kruijssen et al. 2015). Assuming the turbulent dissipation scale is close to the sonic scale with a sound speed $c_{\mathrm{s}}=0.6\left(T_{\mathrm{G}} / 100 \mathrm{~K}\right)^{1 / 2} \mathrm{~km} \mathrm{~s}^{-1}$, the gas temperature will be well mixed on milliparsec spatial scales on kiloyear timescales.

\footnotetext{
15 When averaged into regions in later sections, we measure some temperatures below this limit, albeit still at low significance.

${ }^{16}$ While some emission likely comes from PDRs, we expect these regions to have small filling factors and typically lower densities than cloud centers. At densities $n<10^{4} \mathrm{~cm}^{-3}, \mathrm{p}-\mathrm{H}_{2} \mathrm{CO} 3-2$ emission is very weak and would contribute little to the observed line emission. Volumes within the actively star-forming regions Sgr B2, Sgr C, and near the circumnuclear disk may be locally affected by strong UV heating from young and forming stars, but the bulk of the dense gas is not. Finally, PDR models (e.g., Hollenbach \& Tielens 1999) predict that $T_{\mathrm{D}} \approx T_{\mathrm{G}}$ at high densities, while we show that $T_{\mathrm{G}}>T_{\mathrm{D}}$; see Sect. 5.3.
} 


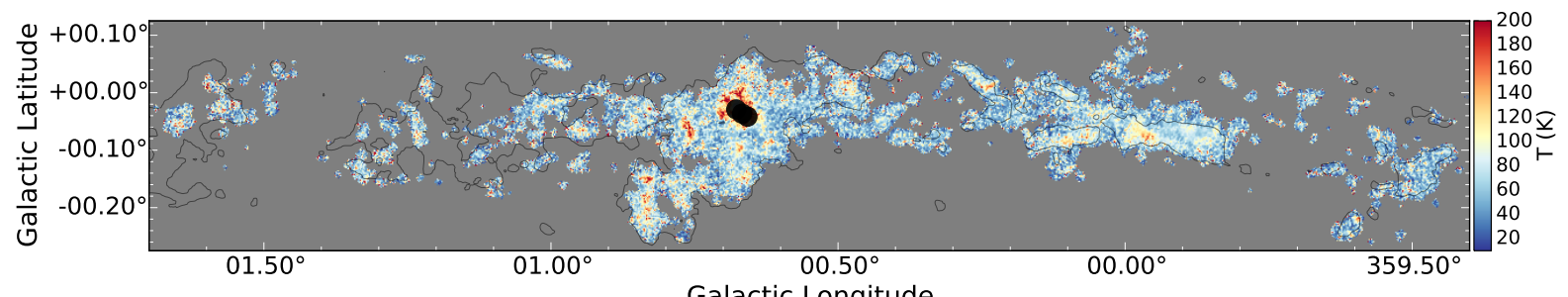

(a)

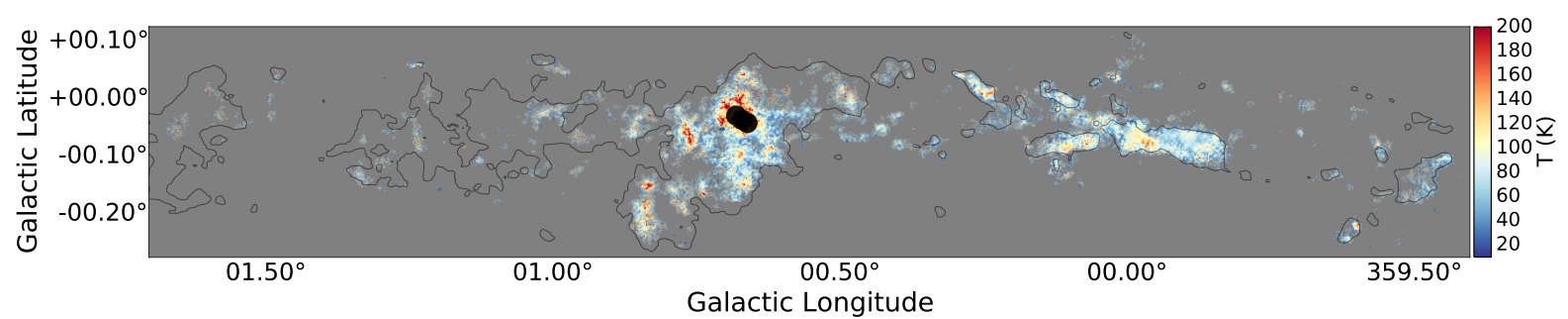

(b)

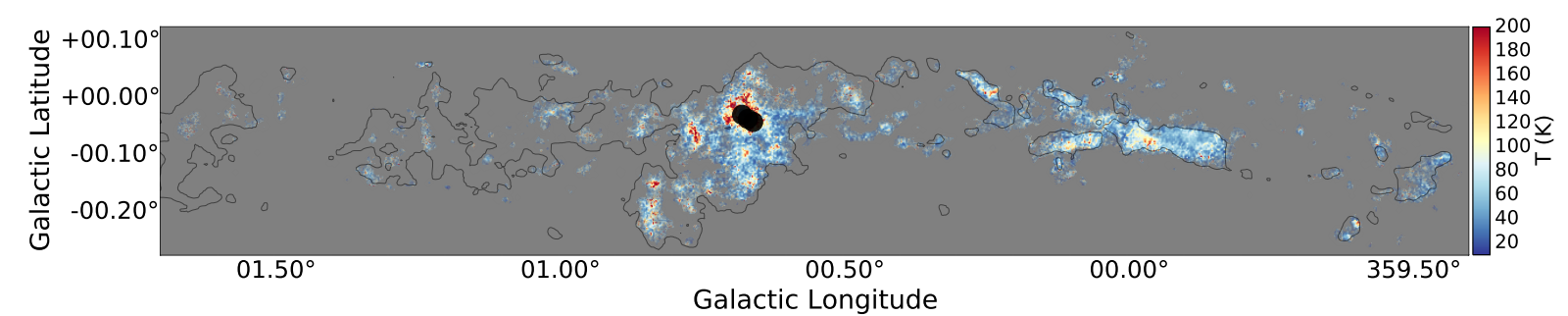

(c)

Fig. 7. Temperature maps assuming fixed $X_{\mathrm{p}-\mathrm{H}_{2} \mathrm{CO}}=1.2 \times 10^{-9}$ and $n\left(\mathrm{H}_{2}\right)$. The maps in b) and c) are masked by signal-to-noise such that regions that have lower signal-to-noise are more gray. The thin black contours are at a column $N\left(\mathrm{H}_{2}\right)=5 \times 10^{22} \mathrm{~cm}^{-2}$ from the Herschel SED fit maps. a) $n\left(\mathrm{H}_{2}\right)=10^{4} \mathrm{~cm}^{-3}$, no mask; b) $n\left(\mathrm{H}_{2}\right)=10^{4} \mathrm{~cm}^{-3}$; c) $n\left(\mathrm{H}_{2}\right)=10^{5} \mathrm{~cm}^{-3}$.

We expand upon the Ao et al. (2013) analysis by solving for the temperature assuming that both cosmic ray ionization and turbulent heating are present and that the dust temperature is nonzero. We assume the heating rate for cosmic rays is constant per cubic centimeter $\Gamma_{\mathrm{CR}}=n \zeta_{\mathrm{CR}}$, and the heating rate from turbulence $\Gamma_{\text {turb }}=n \sigma^{3} / L$, where $\sigma$ is the $3 \mathrm{D}$ velocity dispersion and $L$ is the length scale. We used DESPOTIC (Krumholz 2014) to determine the equilibrium temperature. We included $\mathrm{CO}, \mathrm{O}$, $\mathrm{C}+, \mathrm{C}, \mathrm{o}-\mathrm{H}_{2}, \mathrm{p}-\mathrm{H}_{2}$, and $\mathrm{HD}^{17}$ as line coolants ${ }^{18}$. We used the large velocity gradient (LVG) approximation for optical depth calculation. The resulting curves for $T_{\mathrm{G}}\left(\sigma_{v}\right)$ are shown in Fig. 9 (see Appendix $\mathrm{F}$ for further details). We have assumed a size scale $L=5 \mathrm{pc}$, a gradient $\mathrm{d} v / \mathrm{d} r=5 \mathrm{~km} \mathrm{~s}^{-1} \mathrm{pc}^{-1}$, and a cosmic ray ionization rate (CRIR) $\zeta_{\mathrm{CR}}=10^{-17} \mathrm{~s}^{-1}$, with exceptions noted in the legend. We assume a dust temperature $T_{\mathrm{D}}=25 \mathrm{~K}$,

\footnotetext{
17 The collision rate files for $\mathrm{H}_{2}$ and $\mathrm{HD}$ were provided by Wing-fai Thi from work by the ProDiMo team from Le Bourlot et al. (1999), Wrathmall et al. (2007), Flower et al. (2000), Wolniewicz et al. (1998). Input abundances were $X(\mathrm{CO})=10^{-4}, X\left({ }^{13} \mathrm{CO}\right)=5 \times 10^{-7}, X\left(\mathrm{C}^{18} \mathrm{O}\right)=$ $5 \times 10^{-8}, X(C)=5 \times 10^{-7}, X(C+)=5 \times 10^{-10}, X(O)=5 \times 10^{-6}$, $X(\mathrm{HD})=10^{-5}$, and the ortho/para ratio of $\mathrm{H}_{2} O P R=3$.

${ }^{18}$ We assume a very low abundance for $\mathrm{C}+, X_{C+}=5 \times 10^{-10}$, assuming that cloud shielding will prevent photoionization and collisions with $\mathrm{H}_{2}$ will destroy $\mathrm{CR}$-generated $\mathrm{C}+$ rapidly, which is confirmed by the Nelson \& Langer (1999) chemical model. Lis \& Menten (1998) and Lis et al. (1999) observed bright $\mathrm{C}+$ emission from The Brick; we interpret this emission as coming from a heated outer skin of the cloud at lower densities not coincident with the observed $\mathrm{H}_{2} \mathrm{CO}$ emission.
}

but we assume lower dust radiation temperatures because the dust is optically thin at long wavelengths in most regions. We have assumed a constant interstellar radiation field (ISRF) of $G_{0}$, which should only weakly affect dust temperatures and has no effect on the gas at the high column densities being modeled. A higher ISRF may be appropriate but does not change our results, since a $1000 G_{0}$ field provides the same gas heating rate as a $\zeta_{\mathrm{CR}}=2.5 \times 10^{-17} \mathrm{~s}^{-1}$, a rate much lower than the measured CRIRs in the CMZ. Clark et al. (2013) similarly find that the ISRF has little effect on the gas temperature.

A first key point is that we measure gas temperatures $T_{\mathrm{G}} \sim$ $60 \mathrm{~K}$ in many clouds everywhere throughout the CMZ. Since cosmic rays have very high penetration depths, we expect that cosmic ray heating is uniform everywhere within the CMZ, independent of column density ${ }^{19}$. If this uniformity holds, then we can set an upper limit on the cosmic ray ionization rate, $\zeta_{\mathrm{CR}} \lesssim 10^{-14} \mathrm{~s}^{-1}$, since a higher rate would result in a minimum gas temperature above what we have measured. The limit is consistent with many previous measurements of the CRIR, though it rules out some of the more extreme values (Yusef-Zadeh et al. 2013; Goto et al. 2013). For a fiducial upper limit measurement, we use $n=10^{4} \mathrm{~cm}^{-3}, \mathrm{~d} v / \mathrm{d} r=5 \mathrm{~km} \mathrm{~s}^{-1} \mathrm{pc}^{-1}, T_{\text {rad,D }}=10 \mathrm{~K}$, and

\footnotetext{
${ }^{19}$ However, low-energy cosmic rays will be affected by the strong magnetic fields in the CMZ. Our naive assumptions gloss over much of the physics of cosmic ray interaction with molecular cloud gas, but they are consistent with typical assumptions adopted in the literature and are necessary for a constant cosmic ray ionization rate $\left(\mathrm{a} \zeta_{\mathrm{CR}}\right)$ to be meaningful.
} 


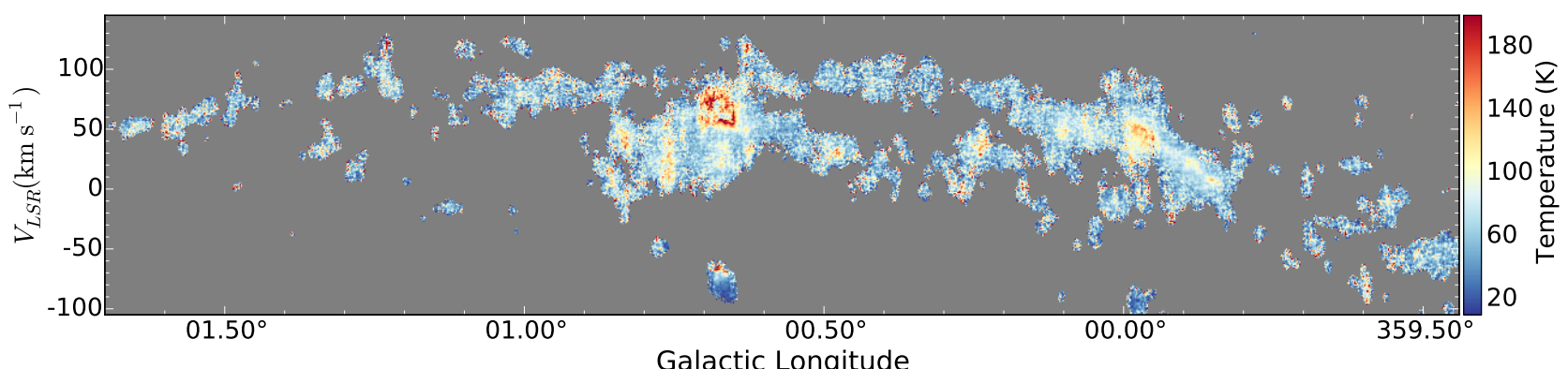

(a)

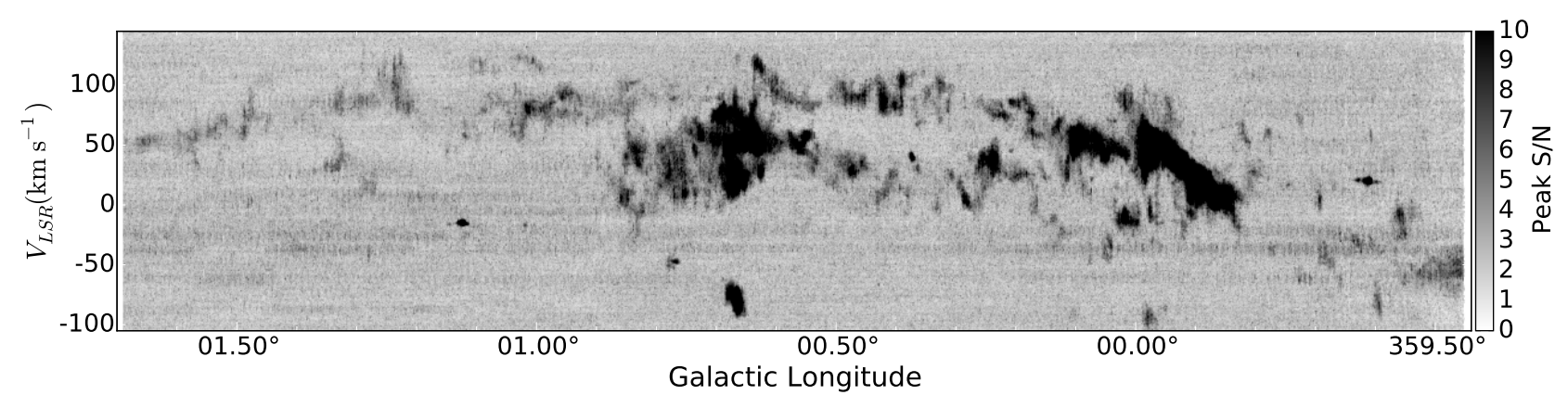

(b)

Fig. 8. a) A Galactic longitude-velocity diagram made by taking the $3_{0,3}-2_{0,2}$-weighted average of the $R_{1}$ PPV cube along Galactic latitude and using the $n=10^{4} \mathrm{~cm}^{-3}$ curve from Fig. 6 to derive temperature. b) A Galactic longitude-velocity map of peak signal-to-noise along Galactic latitude, which is used as a mask in a). The apparently cold clumps at $-80 \mathrm{~km} \mathrm{~s}^{-1}$ in Sgr B2 and $-90 \mathrm{~km} \mathrm{~s}^{-1}$ in Sgr A are from a different line, $\mathrm{HC}_{3} \mathrm{~N}$ 24-23: the gas temperatures from these positions are meaningless. The apparent high temperatures around the cloud edges are artifacts caused by lower signal-to-noise ratios at the edges and should be ignored.

$\zeta_{\mathrm{CR}}=10^{-14} \mathrm{~s}^{-1}$, which yields $T_{\mathrm{G}}=72 \mathrm{~K}$ in the absence of any other heating mechanisms. This upper limit could be violated if the gas in all observed clumps is about an order of magnitude denser than the dust-derived average density, which is not likely. This limit leads to the conclusion that cosmic ray heating is either not dominant in the CMZ or not uniform, as uniform cosmic ray heating cannot satisfy the requirement for both high $(>100 \mathrm{~K})$ and low $(\lesssim 40 \mathrm{~K})$ temperatures to be present in the CMZ.

We have information on the linewidth, size scale, and density for each individual region, albeit with great uncertainties on each. To assess the role of heating by turbulent energy dissipation, we use these measurements to predict the gas temperature. We use the effective radius, which is the geometric mean between the moment-derived major and minor axes ${ }^{20}$, of the selected sources as the length scale, the measured linewidth ${ }^{21} \sigma_{v}$, the measured dust temperature $T_{\mathrm{D}}$ scaled down by an optical depth correction $1-\exp \left[-N\left(\mathrm{H}_{2}\right) / 10^{24} \mathrm{~cm}^{-2}\right]$, and we assume the velocity gradient $\mathrm{d} v / \mathrm{d} r=5 \mathrm{~km} \mathrm{~s}^{-1} \mathrm{pc}^{-1}$. Of these measurements, the linewidth $\sigma_{v}$ is dominant in determining the predicted temperature and is the least uncertain. This analysis is performed both for the hand-selected apertures, which have arbitrary radii but well-measured linewidths, and the dendrogram leaf sources.

\footnotetext{
${ }^{20}$ The effective radius is often computed as the square root of the total area (in pixels), but Rosolowsky \& Leroy (2006) show that this is usually a very bad approximation, so we use the Gaussian-corrected (see next footnote) moment-derived radius.

${ }^{21}$ We correct the linewidth and the radius of dendrogram-extracted clumps for clipping assuming the underlying source is Gaussian following Rosolowsky \& Blitz (2005, Appendix B).
}

The results of the turbulence-predicted temperature analysis are shown in Fig. 10, which plots our measured temperature against the predicted temperature assuming turbulent heating is active and the cosmic ray heating is negligible $\left(\zeta_{\mathrm{CR}}=\right.$ $\left.1 \times 10^{-17} \mathrm{~s}^{-1}\right)$. For the hand-extracted regions, there is no strong trend in the data, but most data points can be explained by turbulent heating alone, perhaps with cosmic rays setting a high floor temperature. There are a few points with measured temperatures below the turbulence prediction. Some of these regions include at least one velocity component that seems to be genuinely cool and broad. A possible explanation for these regions is that their linewidths are an indication of bulk motion rather than turbulent motion, and therefore they may be good candidates for expanding bubble edges, either supernova or $\mathrm{H}$ II-region driven. Alternatively, they may contain denser gas and therefore be able to cool more efficiently; these regions would then be good regions to search for star formation.

The dendrogram-extracted points plotted include only those with a Gaussian correction factor $<3$, as larger corrections are regarded as unreliable extrapolations. Since dendrogram-extracted sources are necessarily coherent in position-velocity space, they are less likely than the hand-selected features (which were selected in position-position space) to represent unassociated line-of-sight features.

The predicted temperatures for dendrogram clumps frequently exceed the measured temperatures by a large margin, often a factor of 2 or more. There are two classes of explanation: first, that the cooling rate has been underestimated. This is entirely plausible, as we have assumed solar neighborhood chemistry (specifically, a CO abundance $X_{\mathrm{CO}}=10^{-4} \mathrm{H}_{2}$, and negligible abundances for other coolants), which does not necessarily hold under CMZ conditions. The other possibility is that we have 

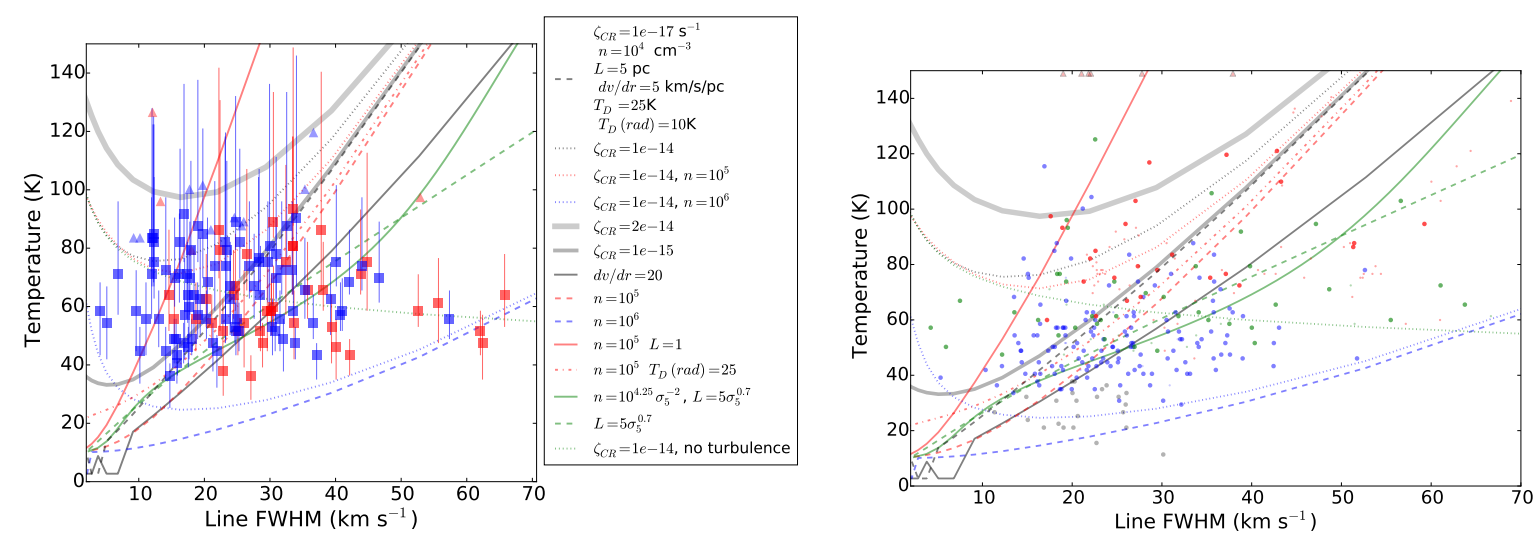

Fig. 9. Gas kinetic temperature vs. linewidth. The lines in both plots show modeled temperatures as a function of line width given different assumptions as computed by DESPOTIC. A version of this plot with no data is shown in Fig. F.1. The legend shows the various assumptions, with the fiducial assumptions indicated at the top, associated with the dashed gray line; these fiducial values are kept fixed except where indicated. The colors of the curves each correspond to a different density, while different line styles indicate changes in other physical constants. The green solid curve shows a pressure equilibrium case $\left(n \propto \sigma^{-2}\right)$ with a Larson relation $\left(L \propto \sigma^{0.7}\right)$ based on Shetty et al. (2012). a) The fitted temperature as described in Sect. 4.2 plotted against the fitted line width for the by-eye selected regions. The blue symbols are compact "clump" sources and the red symbols are large-area $\left(8^{\prime}\right)$ square regions. The very broad line regions, $F W H M \gtrsim 40 \mathrm{~km} \mathrm{~s}^{-1}$, are sometimes affected by significant baseline issues, and it is possible that the temperature has been underestimated as a result (underestimated $3_{2,1}-2_{2,0}$ lines, and therefore underestimated temperatures, are possible because of the lower signal-to-noise of the $3_{2,1}-2_{2,0}$ line; the $3_{0,3}-2_{0,2}$ line is less likely to be affected). b) The same as a) but for the dendrogram-extracted sources. The points are color coded by signal-to-noise in the ratio $R_{1}$, with black $S / N<5$, blue $5<S / N<25$, green $25<S / N<50$, and red $S / N>50$. Small, faint points indicate ancestors in the dendrogram, while bright, large points indicate leaves. Lower limits on linewidth and temperature are indicated with triangles on the edge of the plot. Note that in both panels, any correlation is even weaker than that observed in Fig. 6 of Hüttemeister et al. (1993), who make the same plot for $\mathrm{NH}_{3}(4,4)$ and $(5$, 5). Riquelme et al. (2013) also found no correlation using $\mathrm{NH}_{3}$ over a similar range of velocity dispersions. While there is no clear correlation between velocity dispersion and temperature, most data points fall within a range that is consistent with turbulent heating if the appropriate size scale is selected; see Fig. 10 for plots that account for the differing source sizes. No single cosmic ray ionization rate is capable of reproducing our observations, and the higher CRIRs $\left(\zeta_{\mathrm{CR}}>10^{-14} \mathrm{~s}^{-1}\right)$ are inconsistent with most of the data.

overestimated the line width or underestimated the size scale of the clump via our extraction method. While we have tried to account for this source of error by correcting for clipping, we are nonetheless extrapolating our measurements.

The predicted temperatures for the hand-extracted regions are more uncertain. Most of the hand-extracted regions agree with the prediction at a $1 \sigma$ and all but a few at a $2 \sigma$ level. Given that the sizes used for these regions are uncertain by a large factor (for square apertures especially), there is no significant disagreement between $T_{\mathrm{G} \text {,turb }}$ and $T_{\mathrm{G} \text {,measured }}$.

To explore the possibility that our cooling rates are underestimated, we examine a particular clump from the catalog, with $n=10^{4.2} \mathrm{~cm}^{-3}, \sigma=8.6 f_{\mathrm{g}}=14.3 \mathrm{~km} \mathrm{~s}^{-1}, L=2 r=2(0.588) f_{\mathrm{g}}=$ $1.65 \mathrm{pc}$ (beam-deconvolved), $T_{\mathrm{D}}=21 \mathrm{~K}$, and $T_{\mathrm{D}, \mathrm{rad}}=1.2 \mathrm{~K}$, where $f_{\mathrm{g}}$ is the Gaussian correction factor. This clump has a predicted temperature $T_{\mathrm{G}}=156 \mathrm{~K}$, but a measured temperature $T_{\mathrm{G}}=91 \mathrm{~K}$. With local chemistry, the dominant coolant is $\mathrm{CO}$ by 2 orders of magnitude. In DESPOTIC, we set chemical equilibrium using the Nelson \& Langer (1999) chemical network code and find that the predicted $\mathrm{O}$ abundance is increased by an order of magnitude, which in turn makes oxygen a significant coolant with $\Lambda_{\mathrm{O}} \approx 0.5 \Lambda_{\mathrm{CO}}$, where $\Lambda$ is the cooling rate in erg s $\mathrm{sm}^{-1} \mathrm{~cm}^{-3}$ (other coolants remain negligible). The predicted equilibrium temperature is then $T_{\mathrm{G}}=114 \mathrm{~K}$. While this temperature is still a bit high, this example shows that the modified chemistry at these high temperatures should be accounted for when predicting the gas temperature.

We may be able to further constrain the heating mechanisms using this same data set if more precise velocity structure information is obtained for the clouds. Better velocity structure measurements can be achieved by combining line fitting analysis with systematic clump extraction in order to avoid using the extrapolated correction factors. This analysis is left for a future work.

\subsection{Temperature variation with spatial scale or radius}

Photon-driven heating mechanisms are subject to attenuation, so clouds with significant X-ray or UV heating may have heated shells and cool interiors. Turbulent and cosmic ray heating experience no such effects, but they exhibit a different dependence on gas density than the corresponding cooling mechanisms. We would also expect cooler interiors if the clouds are centrally condensed, since more efficient dust cooling can dominate heating processes at very high densities. If clouds are internally heated by gravitational collapse and star formation, the cloud interior could be warmer than its exterior.

We have examined the radial profiles of temperature and velocity dispersion for selected clouds. We measured the ratio $R_{1}$ and the line width as a function of aperture size by extracting progressively larger circular apertures centered on the same point. For each spectrum extracted, we have fitted the 6-parameter model described in Sect. 4.2 to derive the ratio and linewidth.

Different variations with scale are observed. For example, around "The Brick", the ratio decreases from $\sim 0.55$ at the position $\ell=0.237^{\circ}, b=0.008$, which is coincident with the highest measured temperature in The Brick (within a single spectrum), to $\sim 0.33$ when averaged over a radius $\sim 70^{\prime \prime}$ (Fig. 11a). By contrast, Fig. $11 \mathrm{~b}$ shows a highly turbulent example where the temperature is lowest at the center and higher on larger scales. These observations may imply that two different heating mechanisms, one internally driven (The Brick) and one external (G1.23-0.08), are active within the CMZ. 


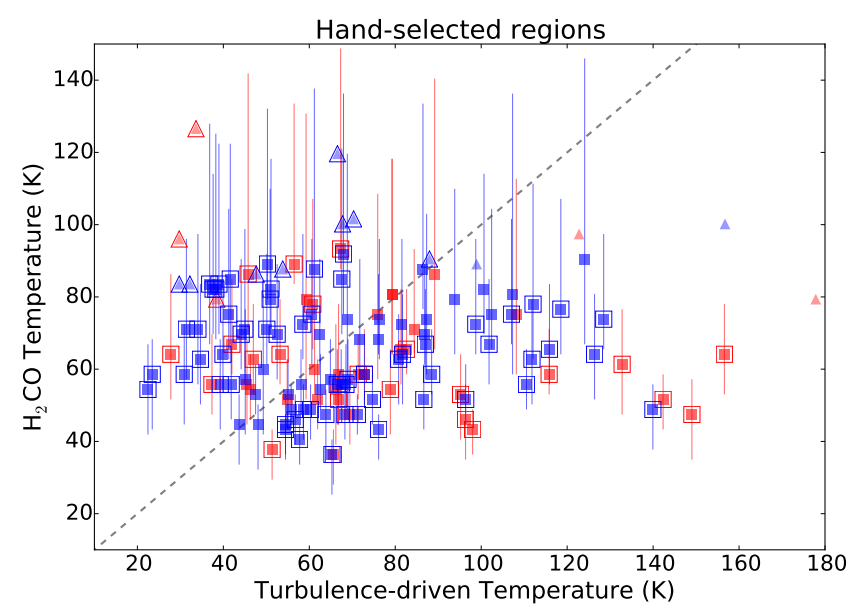

(a)

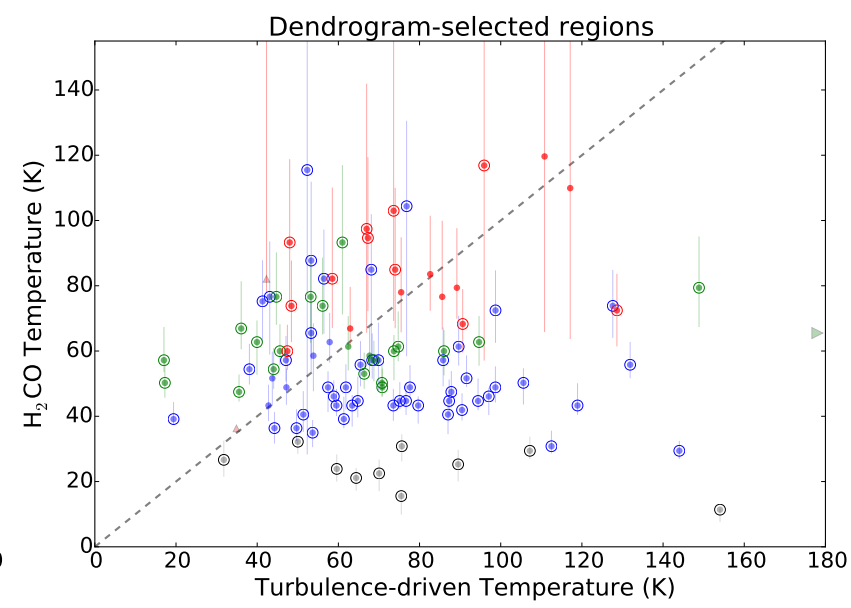

(b)

Fig. 10. $\mathrm{p}-\mathrm{H}_{2} \mathrm{CO}$-measured temperature against the predicted turbulent-driven temperature as discussed in Sect. 5.1. While no trend is obvious in the data, most of the high signal-to-noise data points are fairly close to the $T_{\mathrm{G}, \text { turb }}=T_{\mathrm{G} \text {,measured }}$ line. There are few points with $T_{\mathrm{G} \text {,measured }}>T_{\mathrm{G} \text {,turb }}$, so turbulence generally provides enough - and sometimes too much - energy to heat the gas to the observed temperatures. The regions with predicted temperatures higher than observed require additional explanation: either their heating rate has been overestimated or their cooling rate underestimated. a) The hand-selected regions as described in Fig. 12; the blue symbols are compact "clump" sources and the red symbols are large-area square regions. Triangles indicate regions with lower limits on the measured temperature, i.e. any region with a measured $1-\sigma$ lower limit on the temperature $T_{\mathrm{G}}>150 \mathrm{~K}$. The gray dashed line shows the $T_{\mathrm{G} \text {,turb }}=T_{\mathrm{G} \text {,measured }}$ relation. Regions that are inconsistent with the relation at the $1-\sigma$ level are outlined. b) The same as a) for the dendrogram-extracted regions. Regions with a large Gaussian correction factor $f_{\mathrm{g}}>3$ are excluded. Regions with $T_{\mathrm{G} \text {,turb }}>180 \mathrm{~K}$ are indicated with triangles. The points are color coded by signal-to-noise in the ratio $R_{1}$, with gray $S / N<5$, blue $5<S / N<25$, green $25<S / N<50$, and red $S / N>50$. If the lower signal-to-noise points in blue and gray are ignored, the agreement between the predicted temperature and observed temperature is fairly good.

These two objects are not meant to be representative of the whole CMZ, and the radial properties of clumps need to be studied in more detail to draw firm conclusions about photon-driven heating processes and thermal balance on different size scales.

\subsection{Difference between gas and dust temperatures}

Similar to the results of Ao et al. (2013) for the inner $\sim 75 \mathrm{pc}$, we find that the $\mathrm{p}-\mathrm{H}_{2} \mathrm{CO}$ and dust temperatures are not the same anywhere within the inner $R \lesssim 200$ pc. Figure 12 shows the fitted $\mathrm{p}-\mathrm{H}_{2} \mathrm{CO}$ temperature vs. the fitted dust temperature from HiGal. The majority of the $\mathrm{H}_{2} \mathrm{CO}$ data points are well above the $T_{\mathrm{D}}=T_{\mathrm{G}}$ line independent of the method used to extract the temperature. The few sources that are consistent with $T_{\mathrm{D}}=T_{\mathrm{G}}$ represent an interesting subset of potentially very cool clouds, though they all have low signal-to-noise ratios in the present data set. Overall, though, our data clearly indicate that, on $\sim$ pc scales in the CMZ, dust is a coolant and the gas and dust are not in equilibrium. This disagreement means that gas temperatures are inconsistent with PDR models (Hollenbach \& Tielens 1999) and confirms that UV heating is not a significant driver of the gas temperatures.

\subsection{Spatial variations in gas temperature and orbit comparison}

There are significant spatial variations in the gas temperature. The most obvious is in and around the Sgr B2 complex, where very high gas temperatures are measured. High gas temperatures, $T_{\mathrm{G}} \sim 100-120 \mathrm{~K}$, were previously measured in a ridge to the Galactic northeast of Sgr B2 N using $\mathrm{CH}_{3} \mathrm{CN}$ (de Vicente et al. 1997, Fig. 4b), which we now confirm, though our data show $T_{\mathrm{G}} \gtrsim 150 \mathrm{~K}$. Ott et al. (2014) also showed relatively high
$\mathrm{NH}_{3}$ temperatures in this hot ridge, and Etxaluze et al. (2013) found a high-temperature component $(\sim 50-84 \mathrm{~K})$ in their high- $J$ $\mathrm{CO}$ spectral line energy distribution analysis. The Brick, Sgr A, and the $50 \mathrm{~km} \mathrm{~s}^{-1}$ and $20 \mathrm{~km} \mathrm{~s}^{-1}$ clouds exhibit the highest temperatures outside of Sgr B2, with $T_{\mathrm{G}}>100 \mathrm{~K}$, in agreement with measurements in high- $J$ transitions of $\mathrm{NH}_{3}$ by Mills \& Morris (2013). The western portion of the CMZ, around G359.5, shows cooler temperatures $40 \mathrm{~K}<T_{\mathrm{G}}<60 \mathrm{~K}$. Along the "dust ridge" (see Sect. 1), the high-latitude portion is slightly warmer than the low-latitude region; this difference is most evident when comparing The Brick to the region just south of it and at $\ell=0.5^{\circ}$. Figure 13 shows the temperature of dendrogram clumps as a function of longitude. The high temperatures $\left(T_{\mathrm{G}} \gtrsim 60 \mathrm{~K}\right)$ persist to high longitudes, including the enigmatic turbulent yet starless G1.6 region (Menten et al. 2009).

In order to search for explanations for the temperature variations we observe, we compared our data to predicted cloud orbits. Figure 14 shows the orbital fit from Kruijssen et al. (2015) and a position-velocity slice through the fitted temperature cube following that orbit. The orbit does not align with all cloud components, especially along the green segment, but is still very useful for tracking the properties of clouds in common environments. Assuming we can use the orbit PPV predictions to locate the clouds in 3D, we can begin to understand the geometry of the CMZ. It appears that the far-side clouds (green segment, $1 \mathrm{Myr}<t<2 \mathrm{Myr}$ ), are slightly cooler than many of those on the near side, such as "The Brick" (red segment).

We assigned a time to each dendrogram-extracted clump within $35 \mathrm{~km} \mathrm{~s}^{-1}$ and $6 \mathrm{pc}$ of the Kruijssen et al. (2015) orbit and plotted their temperatures against that time in Fig. 15. We observed a hint of a linear increase in temperature with time at the first crossing. The possible linear increase in temperature over the first $\sim 0.5 \mathrm{Myr}$ would support the Kruijssen et al. (2015) hypothesis that the "ring" represents a single gas stream 


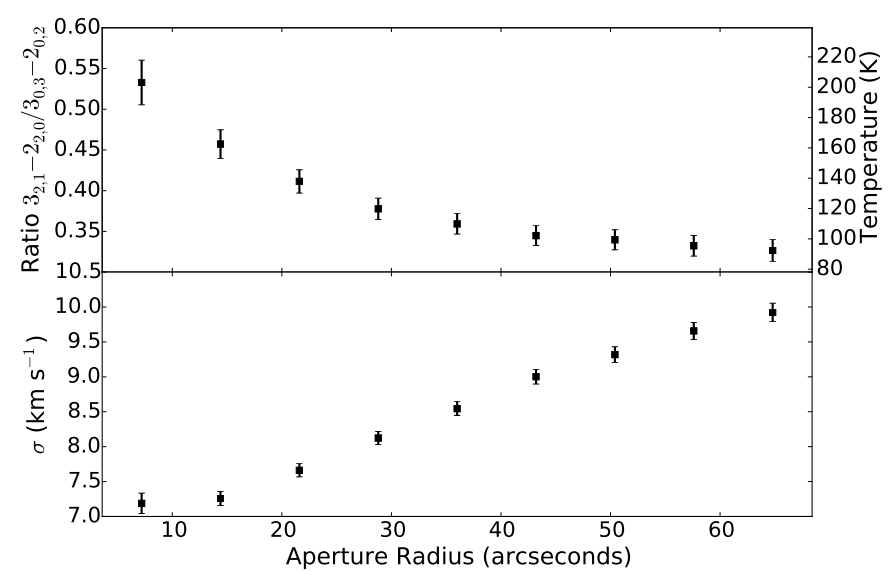

(a)

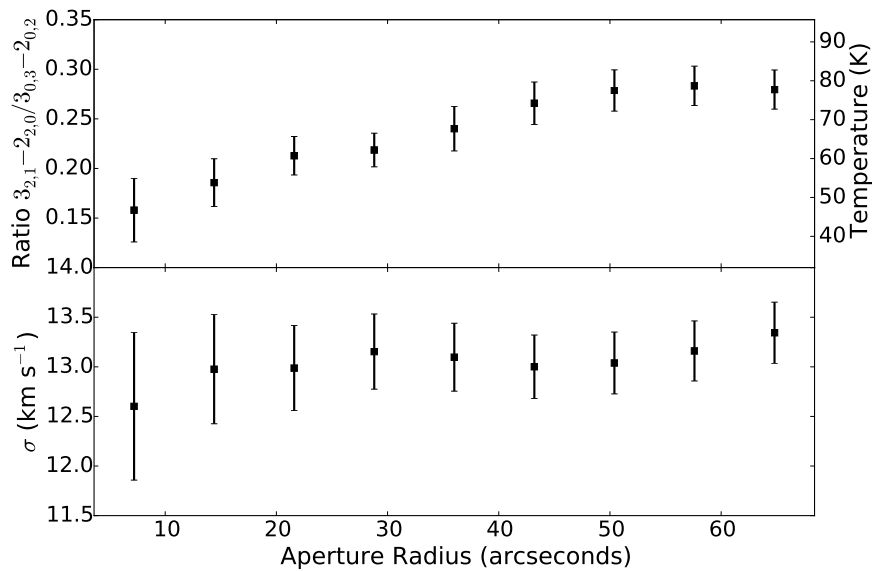

(b)

Fig. 11. a) Radial plots centered on the southwest portion of "The Brick". The top panel shows the ratio p- $\mathrm{H}_{2} \mathrm{CO} S_{v}\left(3_{2,1}-2_{2,0}\right) / S_{v}\left(3_{0,3}-2_{0,2}\right)$ as a function of aperture size, starting from a single pixel. The derived temperature using Fig. 6 is shown on the right axis; temperatures $\gtrsim 150 \mathrm{~K}$ should be regarded as lower limits. The bottom panel shows the line width $\sigma=F W H M / 2.35$ as a function of aperture size. b) The same as a) but for a region centered on G1.23-0.08, a local peak in the $\mathrm{p}-\mathrm{H}_{2} \mathrm{CO} 3_{0,3}-2_{0,2}$ emission. In this case, the ratio $\mathrm{p}-\mathrm{H}_{2} \mathrm{CO} S_{v}\left(3_{2,1}-2_{2,0}\right) / S_{v}\left(3_{0,3}-2_{0,2}\right)$ is lower at the center and increases toward larger radii.
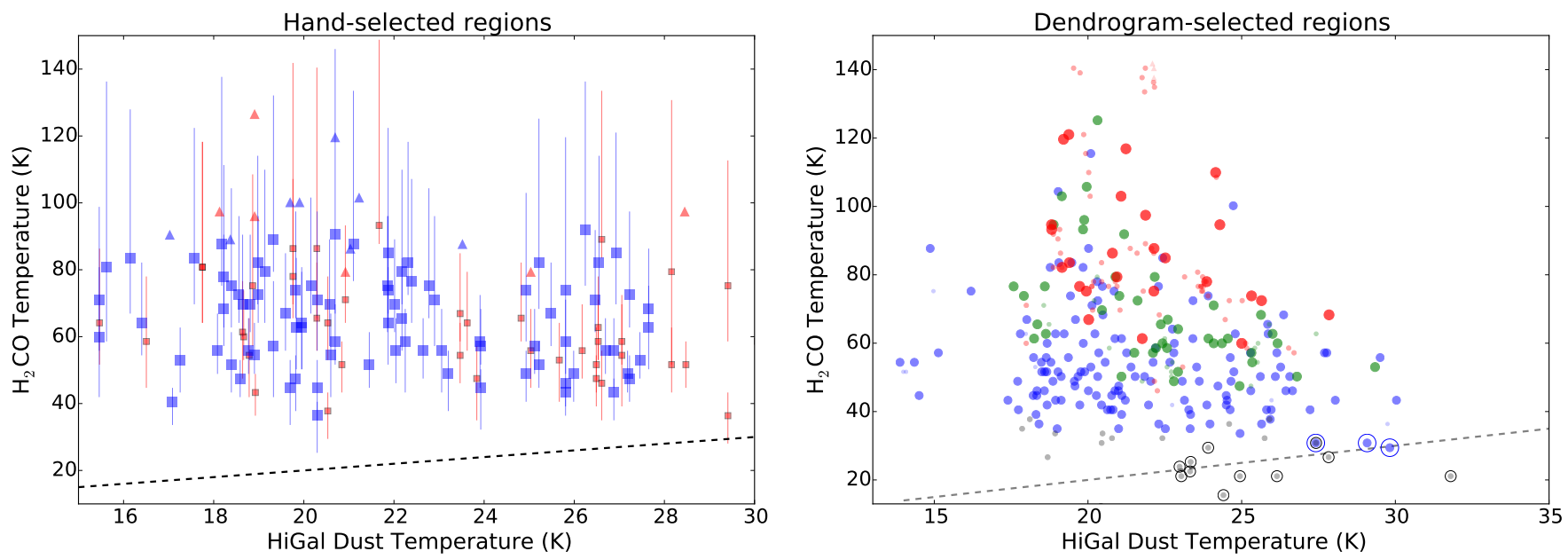

Fig. 12. a) Fitted temperature of region-averaged spectra as described in Sect. 4.2 plotted against the HiGal fitted dust temperature. As in Fig. 9, the blue symbols are compact "clump" sources and the red symbols are large-area square regions. The black dashed line shows the $T_{\mathrm{G}}=T_{\mathrm{D}}$ relation. Nearly all of the data points fall above this relation, and very few are consistent with it at the $3 \sigma$ level. b) Same as a) but for the dendrogramextracted sources, showing a similar discrepancy between dust and gas temperature. The points are color coded by signal-to-noise in the ratio $R_{1}$, with gray $S / N<5$, blue $5<S / N<25$, green $25<S / N<50$, and red $S / N>50$.

following an eccentric orbit with regular pericenter passages by Sgr A*. In this model, the pericenter passage drives additional turbulence and induces collapse, which implies increased turbulent energy dissipation (hence heating) after the pericenter passage. However, the possible trend is also reasonably consistent with a constant temperature over the same range, so we caution against overinterpretation.

\subsection{Comparison to $\mathrm{NH}_{3}$ measurements from Ott et al. (2014)}

Ott et al. (2014) measured the $\mathrm{NH}_{3}(1,1)$ and $(2,2)$ lines over a subset of the region we mapped, from $-0.2^{\circ}$ to $0.8^{\circ}$. These two lines are a frequently used thermometer sensitive to gas temperatures $5 \lesssim T_{\mathrm{G}} \lesssim 40 \mathrm{~K}$, with sensitivity up to $\sim 80 \mathrm{~K}$ but requiring a larger $T_{\text {rot }}$ to $T_{\text {kin }}$ correction, which means the uncertainties at high temperatures are larger (Mangum et al. 2013, their Fig. 1). The $\mathrm{NH}_{3}$ lines have lower critical densities than the $\mathrm{H}_{2} \mathrm{CO} 3-2$ transitions by a few orders of magnitude, $n_{\text {crit }}\left(\mathrm{NH}_{3}(1,1)\right) \sim 1 \times 10^{3} \mathrm{~cm}^{-3}$ while $n_{\text {crit }}\left(\mathrm{p}-\mathrm{H}_{2} \mathrm{CO} 3_{0,3}-2_{0,2}\right) \sim 8 \times 10^{5} \mathrm{~cm}^{-3}$ and $n_{\mathrm{eff}}\left(\mathrm{NH}_{3}(1,1)\right) \sim$ $1 e 3$ while $n_{\text {eff }}\left(\mathrm{p}-\mathrm{H}_{2} \mathrm{CO} 3_{0,3}-2_{0,2}\right) \sim 1 \times 10^{5}$ (Shirley 2015) $)^{22}$. The energy levels of the $3_{0,3}-2_{0,2}$ and $3_{2,1}-2_{2,0}$ lines are 21 and $68 \mathrm{~K}$ respectively, while $\mathrm{p}-\mathrm{NH}_{3}(1-1),(2-2)$, and (4-4) have $E_{\mathrm{U}}=1,42$, and $178 \mathrm{~K}$; the (4-4) line is therefore required to accurately measure temperatures over the range covered by the $\mathrm{p}-\mathrm{H}_{2} \mathrm{CO} \mathrm{J}=3-2$ lines.

The temperatures derived from the $\mathrm{NH}_{3}$ line ratio may be lower for a variety of reasons. The lower critical density means

${ }^{22} n_{\text {crit }}$ is defined to be the ratio between the spontaneous decay coefficient, the Einstein $A_{\mathrm{ul}}$ value, to the sum of the collision rates into and out of the upper level. $n_{\text {eff }}$ is the density required to provide a $1 \mathrm{~K} \mathrm{~km} \mathrm{~s}^{-1}$ flux density given a temperature and a reference column density. For either density measure, the species with the higher $n$ will trace denser gas. 


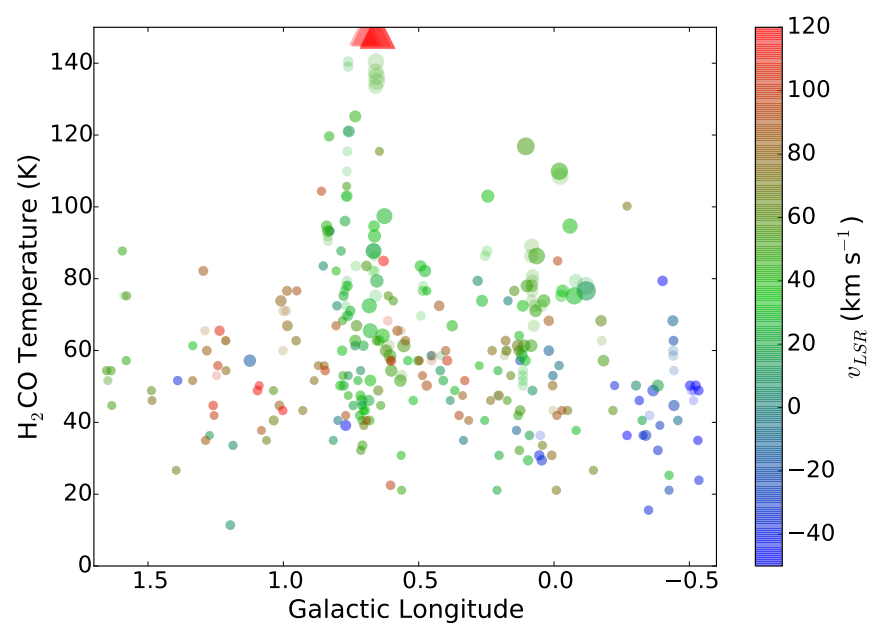

Fig. 13. Derived temperature as a function of longitude colored by LSR velocity for dendrogram-extracted data. The points have a size proportional to the $3_{0,3}-2_{0,2}$ line brightness. The more transparent points reflect ancestors in the dendrogram tree, while the opaque points show leaves. Lower limits for some of the Sgr B2 regions are shown as red upward-pointing triangles near the top of the plot.

that $\mathrm{NH}_{3}$ is measuring more diffuse gas (which is apparently colder) that is not detected at all in $\mathrm{H}_{2} \mathrm{CO}$. Since $\mathrm{H}_{2} \mathrm{CO}$ only provides measurable temperatures above $\sim 50 \mathrm{~K}$ in most locations, there may be low-column gas that was too faint to be measured with $\mathrm{H}_{2} \mathrm{CO}$. However, since Güsten et al. (1981) found temperatures comparable to the $\mathrm{H}_{2} \mathrm{CO}$ temperatures using higher transitions of $\mathrm{NH}_{3}$, there are either multiple temperature components in the diffuse gas or peculiar excitation effects affecting one or both of the thermometers (Güsten et al. 1981 and Morris et al. 1973 suggest that collisional excitation of the $(2,1)$ level may de-populate the $(2,2)$ level of $\mathrm{NH}_{3}$, for example). The presence of more diffuse gas traced by $\mathrm{H}_{3}^{+}$at $T_{\text {gas }} \sim 350 \mathrm{~K}$ (Goto et al. 2011, 2014) suggests that a cold and diffuse component traced by low- $\mathrm{J} \mathrm{NH}_{3}$ is unlikely.

Figure 16 shows the Ott et al. (2014) temperature map in the same color scheme that we have used in Fig. 7. There are some trends that are common between the two maps: the Sgr A complex and Sgr B2 complex are the warmest regions, the diffuse gas surrounding these is somewhat cooler, and the positive latitude component of the Molinari et al. (2011) "ring" is slightly warmer than the negative latitude component.

The most significant difference occurs in the southwest portion of the Sgr B2 cloud. In this region, the $\mathrm{NH}_{3}$ temperature ranges from the high $\left(T_{\mathrm{G}}>80 \mathrm{~K}\right)$ temperatures of the Sgr B2 N hot ridge into a cooler background $T_{\mathrm{G}} \sim 30-40 \mathrm{~K}$. By contrast, the $\mathrm{H}_{2} \mathrm{CO}$ temperatures stay high, indicating gas temperatures $T_{\mathrm{G}}>150 \mathrm{~K}$ extending to the southwest of Sgr B2 S. This difference probably indicates that there is a cool, diffuse component being detected in $\mathrm{NH}_{3}$, while $\mathrm{H}_{2} \mathrm{CO}$ is detecting a hotter, denser component. This region will provide a good comparison target for other thermometric observations.

Further comparison of the $\mathrm{NH}_{3}$ and $\mathrm{H}_{2} \mathrm{CO}$ thermometers is necessary, and will be possible with future ATCA and GBT surveys of higher ammonia transitions. These will be aided by observations of both higher and lower $\mathrm{H}_{2} \mathrm{CO}$ transitions (Mangum $\&$ Wootten 1993) to verify the presence of a cool, diffuse component.

\subsection{Comparison to other measurements with $\mathrm{p}-\mathrm{H}_{2} \mathrm{CO}$}

The largest survey of $\mathrm{p}-\mathrm{H}_{2} \mathrm{CO}$ temperatures prior to this work was by Ao et al. (2013), who observed the inner 75 pc. They performed a similar analysis to ours but over a smaller area. They reported similar temperatures, with some small differences due to a different set of collision rates adopted (we used Wiesenfeld \& Faure 2013, they used Green 1991). The Ao et al. (2013) survey found uniformly high temperatures within the bright Sgr A cloud complex. Our survey finds a greater variety in gas temperatures due to the larger area covered.

There have also been observations of $\mathrm{p}-\mathrm{H}_{2} \mathrm{CO}$ temperatures with interferometers. Johnston et al. (2014) noted a very high temperature peak, $T_{\mathrm{G}}>320 \mathrm{~K}$, using these lines of $\mathrm{p}-\mathrm{H}_{2} \mathrm{CO}$ toward The Brick, G0.253+0.016. The ratio of the SMA to APEX measurements of both the $3_{0,3}-2_{0,2}$ and $3_{2,1}-2_{2,0}$ line in the southwest portion of The Brick is $\approx 0.25$, but the ratio $R_{1} \approx 0.45$ is approximately the same within the $r=25^{\prime \prime}$ aperture we used. The high temperature in Johnston et al. (2014) comes from smaller regions in which a higher $R_{1} \approx 0.71$ is observed.

Above $300 \mathrm{~K}$, the collision rates provided by LAMDA (Green 1991; Schöier et al. 2005; Wiesenfeld \& Faure 2013) must be extrapolated, which means the models do not produce reliable results. Very high $R_{1}$ values above about 0.7 cannot be reproduced by high temperatures with the current suite of collision rates if the temperature is bounded to $T_{\mathrm{G}}<300 \mathrm{~K}$, but instead require that the lines be optically thick and thermalized and therefore that the gas be at very high densities. Given the low brightness temperatures observed $\left(\sim 2 \mathrm{~K}\right.$ even in the SMA $\sim 4^{\prime \prime}$ beams), if the emission is optically thick, it must be coming from an extremely small area (it must have a small filling factor). A small filling factor for the cloud-scale APEX observations is inconsistent with the observed large extent of the emission and the low interferometer to single-dish ratio. We discuss this topic further in Sect. 5.7. We conclude, therefore, that a lower limit on the gas temperature in The Brick, $T_{\mathrm{G}} \gtrsim 120 \mathrm{~K}$, is consistent with our observations, and that the Johnston et al. (2014) high $R_{1}$ measurement should be regarded as either a lower limit $T_{\mathrm{G}} \gtrsim 300 \mathrm{~K}$ or a suggestion that there is sub-arcsecond optically thick p- $\mathrm{H}_{2} \mathrm{CO} 3_{0,3}-2_{0,2}$ emission at the brightness peaks.

\subsection{Is the gas warm or just optically thick?}

As noted in Sect. 5.6, there is degeneracy in the modeling that allows the observed ratios to be produced by very dense gas in addition to hot gas. Indeed, ratios approaching $R_{1}=1$ occur for relatively low temperatures $\left(T_{\mathrm{G}} \sim 30 \mathrm{~K}\right)$ at very high densities $\left(n>10^{6} \mathrm{~cm}^{-3}\right)$ and very high column densities $\left(\mathrm{N}\left(\mathrm{p}-\mathrm{H}_{2} \mathrm{CO}\right)>\right.$ $10^{16}$ per $\mathrm{km} \mathrm{s}^{-1} \mathrm{~cm}^{-2}$ ) because the lines become optically thick and have LTE excitation temperatures.

Since the mean densities at $30^{\prime \prime}$ resolution are always significantly lower $\left(n \lesssim\right.$ few $\left.\times 10^{5} \mathrm{~cm}^{-3}\right)$, such high-density, optically thick gas would have to have a very low filling factor. The peak $\mathrm{p}-\mathrm{H}_{2} \mathrm{CO} 3_{0,3}-2_{0,2}$ brightness ranges from $0.2 \lesssim T_{\mathrm{mb}} \lesssim 2 \mathrm{~K}$ in the detected regions, implying a filling factor upper limit $f f \lesssim 1-7 \%$ if we assume the resolved brightness temperature equals the dust temperature, $T_{\mathrm{B}}=T_{\mathrm{D}}$. In practice, the filling factor would have to be lower still, since any optically thin $\mathrm{p}-\mathrm{H}_{2} \mathrm{CO}$ in surrounding lower-density gas would favor the $3_{0,3}-2_{0,2}$ line and contribute to a lower $R_{1}$.

The strongest argument against an optically thick, cold gas explanation for our observations is the extremely high column density required. At typical $\mathrm{p}-\mathrm{H}_{2} \mathrm{CO}$ abundances $X \sim 10^{-9}$, or even more extreme abundances $X \sim 10^{-8}$, the implied local 


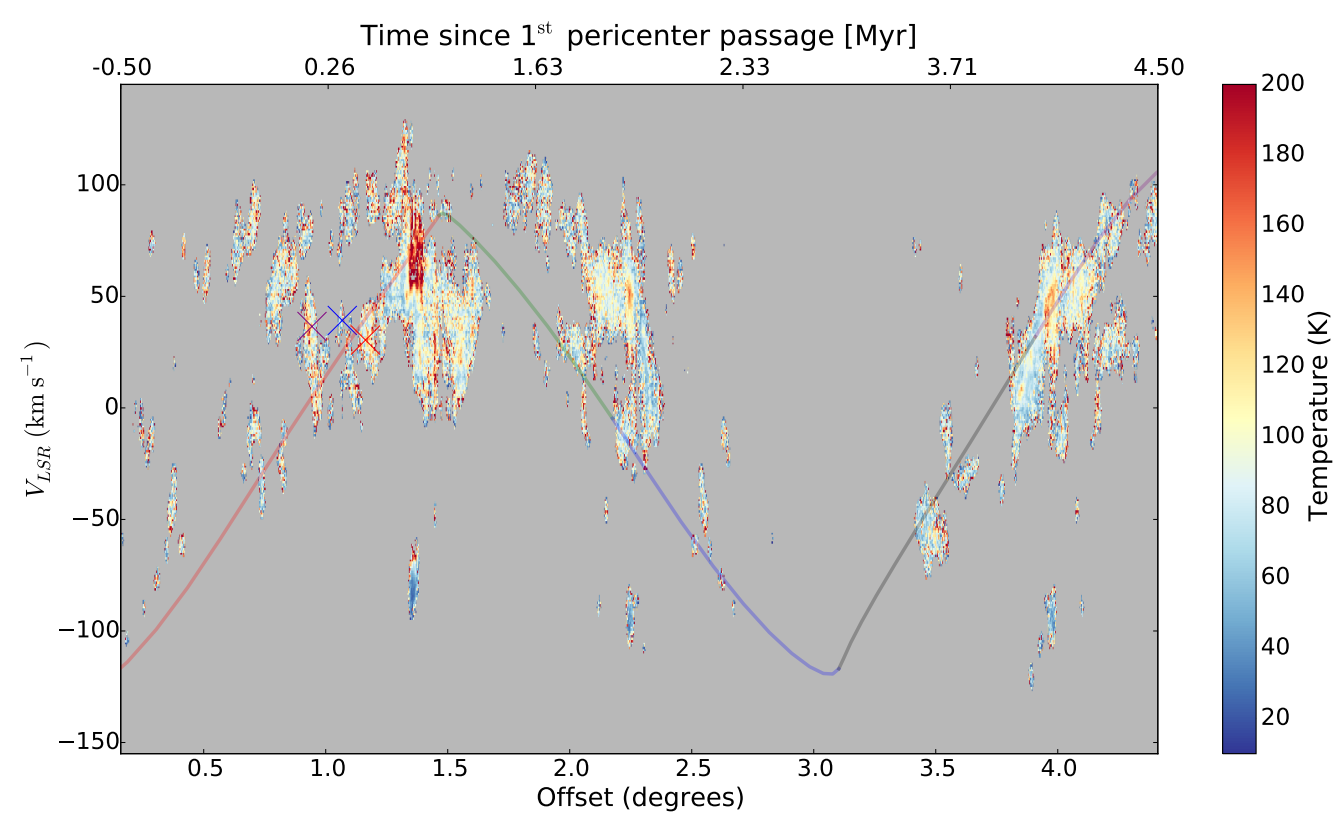

(a)

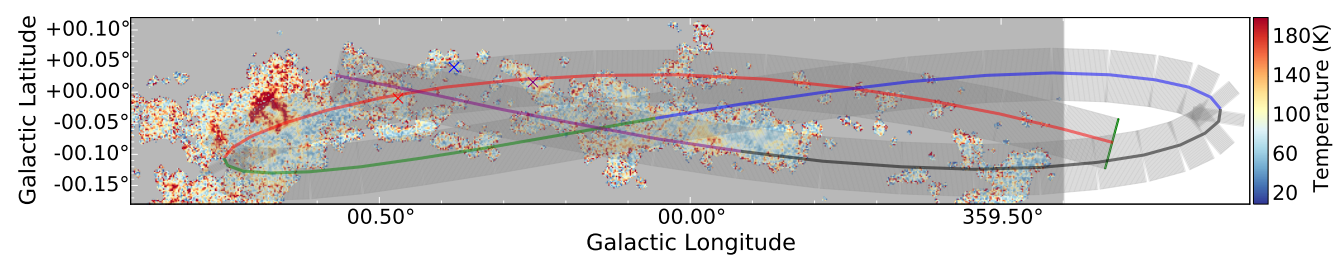

(b)

Fig. 14. a) A position-velocity slice through the temperature cube along the Kruijssen et al. (2015) orbit. Gray regions have no gas detected or inadequate signal to measure a temperature. b) The path used to create the slice shown in a). The path consists of a series of transparent $7.2^{\prime \prime} \times 300^{\prime \prime}$ rectangles over which the temperature has been averaged. The green bar at $\ell=359.3^{\circ}$ indicates the starting point (offset $\left.=0\right)$ of the positionvelocity slice. The orbit is outside of our observed field to the west of Sgr C. The X's mark The Brick (purple), cloud d (blue), and cloud e (red) to provide landmarks for comparison between the two figures.

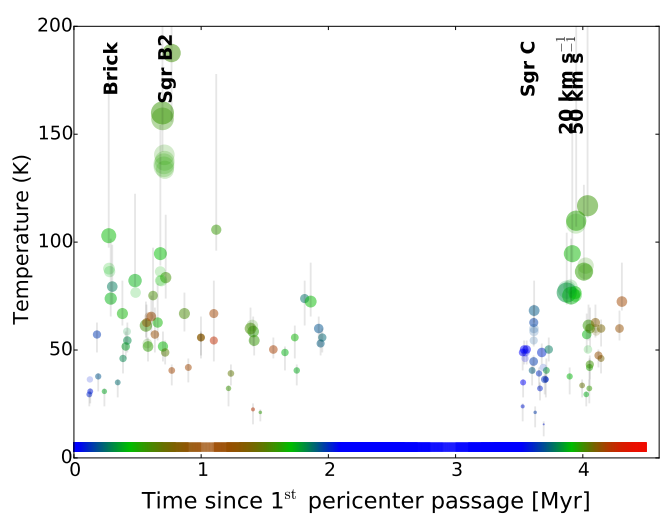

(a)
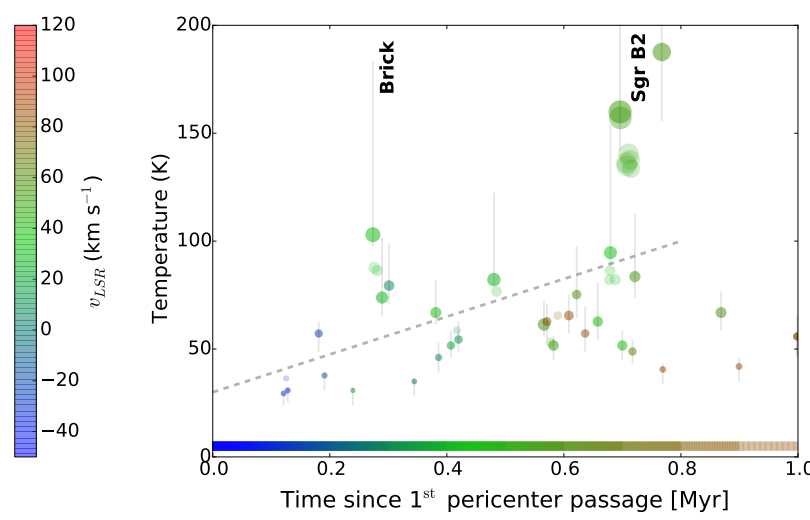

(b)

Fig. 15. Observed temperature vs. time since the most recent pericenter passage of The Brick and the dust ridge clouds on the Kruijssen et al. (2015) orbit for dendrogram-extracted clumps within $35 \mathrm{~km} \mathrm{~s}^{-1}$ and $12 \mathrm{pc}$ of the orbital path. a) shows the entire orbit while b) shows an expanded view of the first Myr. The colored bar along the bottom of the figure shows the modeled orbit velocity at each position. In both panels, the darker symbols represent "leaves" in the dendrogram structure, i.e. compact clumps, while the fainter symbols represent their parent structures. There is a hint of an increase in temperature with time, from an initial temperature $T_{\mathrm{G}} \lesssim 50 \mathrm{~K}$ to $T_{\mathrm{G}} \gtrsim 100 \mathrm{~K}$ over a period $\tau \sim 0.8 \mathrm{Myr}$, as illustrated by the dashed line in panel b); note that the cool features from 0.7-0.9 Myr are at a significantly different velocity and therefore cannot be part of the same stream. The Brick is an outlier (it is hotter) whether or not there is a trend. The symbol sizes are proportional to the p- $\mathrm{H}_{2} \mathrm{CO} 3_{2,1}-2_{2,0}$ brightness and are meant to give an indication of how reliable the temperature measurements are. The gray vertical lines through the circles indicate the formal $1 \sigma$ errors on the temperature. 


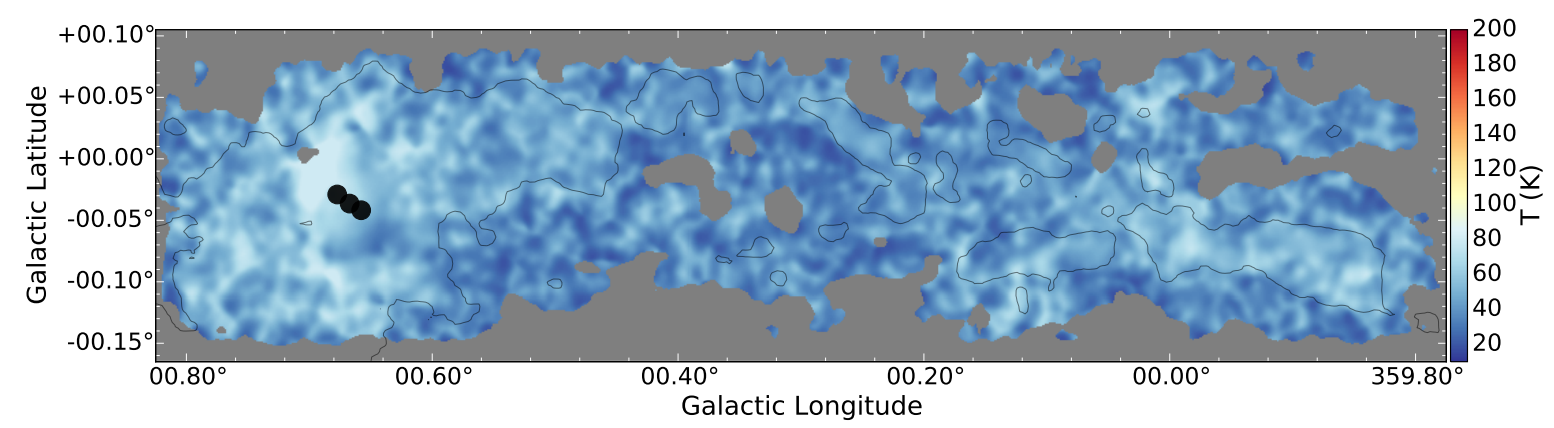

(a)

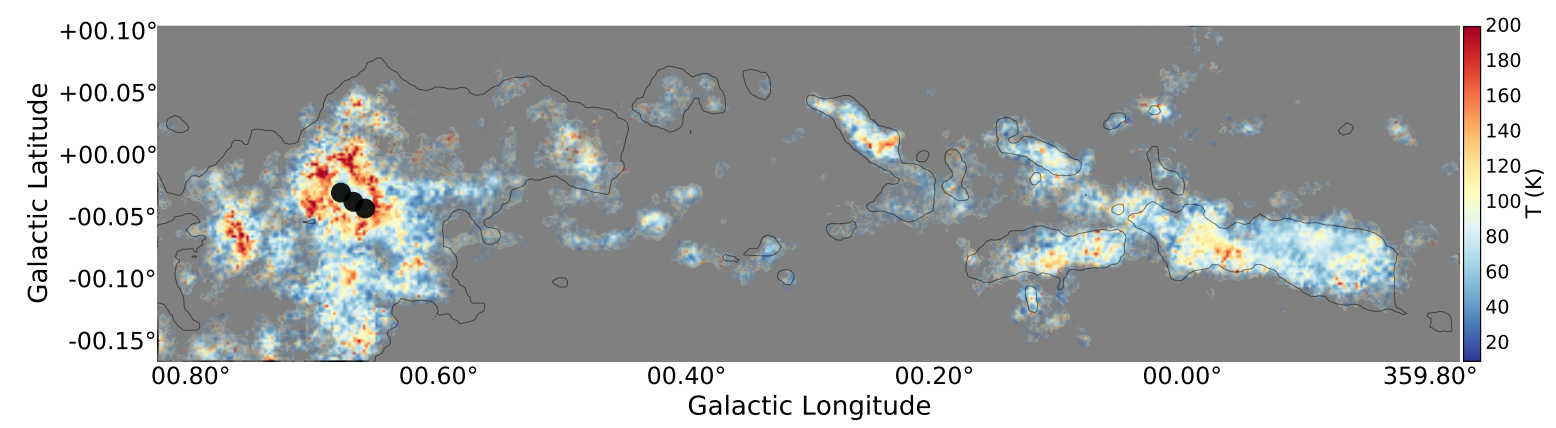

(b)

Fig. 16. a) A temperature map derived from the $\mathrm{NH}_{3}(1,1) /(2,2)$ line ratio from Ott et al. (2014). The $\mathrm{NH}_{3}$ thermometer has a more limited temperature range when using only these two lines, so the map is cut off at $T_{\mathrm{G}}=80 \mathrm{~K}$. b) The $\mathrm{p}-\mathrm{H}_{2} \mathrm{CO}$-derived temperature map over the same region from Fig. 7a.

column density is $N\left(\mathrm{H}_{2}\right) \gtrsim 10^{25(24)} \mathrm{cm}^{-2}$ in a single velocity bin. Column densities up to $N\left(\mathrm{H}_{2}\right) \sim 10^{24} \mathrm{~cm}^{-2}$ are observed, but with much lower filling fractions $f f \sim 10^{-4}$, in The Brick (Rathborne et al. 2014, their Fig. 4). Even in The Brick, one of the densest clouds in the dust ridge besides Sgr B2, higher column densities are not observed. So, while it remains possible that optically thick $\mathrm{p}-\mathrm{H}_{2} \mathrm{CO}$ is responsible for the observed line ratios, it is very unlikely, especially outside the densest clouds.

One remaining possibility is that the $\mathrm{p}-\mathrm{H}_{2} \mathrm{CO}$ lines become optically thick because of dramatically reduced turbulent line spreading and therefore increased line trapping. Such an effect would require a transition from supersonically turbulent to coherent (subsonically turbulent) gas motion (e.g., Pineda et al. 2010 ) and could reduce the required column densities by $\sim 100 \times$. Kauffmann et al. (2013) observed a few clumps within The Brick with narrow velocity dispersions that could represent subsonic regions, and such clumps could in principle mimic the observed signal since their relative line-of-sight velocities are high. However, there are not enough of these to account for the observed line brightness, and there were also equally many high-velocity-dispersion clumps detected.

A related argument is that the high temperatures come from a very small fraction of the gas heated in high velocity shocks, i.e. that we are seeing intermittent high temperatures (e.g., Falgarone \& Puget 1995). Given the high observed velocity dispersions, high velocity shocks capable of heating the gas far above the observed temperatures are possible. Very high velocity shocks, with $v_{\mathrm{s}} \gtrsim 50 \mathrm{~km} \mathrm{~s}^{-1}$ will dissociate molecules, so in this scenario the emission must come from intermediate velocity shocks (Neufeld \& Dalgarno 1989). The primary distinction between this intermittent model and the more generic turbulent dissipation heating model is timescale: in the intermittent model, the shock-heated gas is not well-mixed with the rest of the gas and therefore represents a distinct phase.

\subsection{The $C M Z$ average}

For comparison to extragalactic observations, we have included a spectrum averaged over the whole $\sim 300$ pc extent of our survey in Fig. 17. The peak amplitude is $50-60 \mathrm{mK}$ and the measured line ratio $\mathrm{p}-\mathrm{H}_{2} \mathrm{CO} S_{v}\left(3_{2,1}-2_{2,0}\right) / S_{v}\left(3_{0,3}-2_{0,2}\right)=0.25$, corresponding to a temperature $T_{\mathrm{G}}=65 \mathrm{~K}$ at $n=10^{4} \mathrm{~cm}^{-3}$, $T_{\mathrm{G}}=63 \mathrm{~K}$ at $n=10^{4.5} \mathrm{~cm}^{-3}$, or $T_{\mathrm{G}}=48 \mathrm{~K}$ at $n=10^{5} \mathrm{~cm}^{-3}$. Over the same area, the measured mean dust temperature from HiGal is $23 \mathrm{~K}$, though on such large scales it would be more appropriate to use Planck or WMAP data to perform this measurement. While the spectral fit does not capture all components of the line, the extracted line ratio is representative of the CMZ-wide average. This temperature is very close to the $\mathrm{NH}_{3}$-measured temperature in M83 (56 K; Mangum et al. 2013), which has a similarly low dust temperature $T_{\mathrm{D}, \mathrm{M} 83}=31 \mathrm{~K}$ over a 600 pc region $^{23}$. Maffei 2, NGC 1365, and NGC 6946 also exhibit similar gas and dust temperatures to our Galactic center in the Mangum et al. (2013) sample, suggesting that these galaxies all have analogous gas thermal structures in their inner regions. The other galaxies in that sample, especially the starburst galaxies, have significantly warmer gas and dust, with temperatures closer to those measured for Sgr B2 than for the whole CMZ.

${ }^{23}$ We also examine the HOPS (Walsh et al. 2011) $\mathrm{NH}_{3}$ observations of the $\mathrm{CMZ}$ over the same region as our $\mathrm{H}_{2} \mathrm{CO}$ survey, and find that the measured $(1-1) /(2-2)$ ratio is 1.48 , identical to that measured for M83 to within measurement errors, so the ammonia temperature on these large scales is also consistent. 


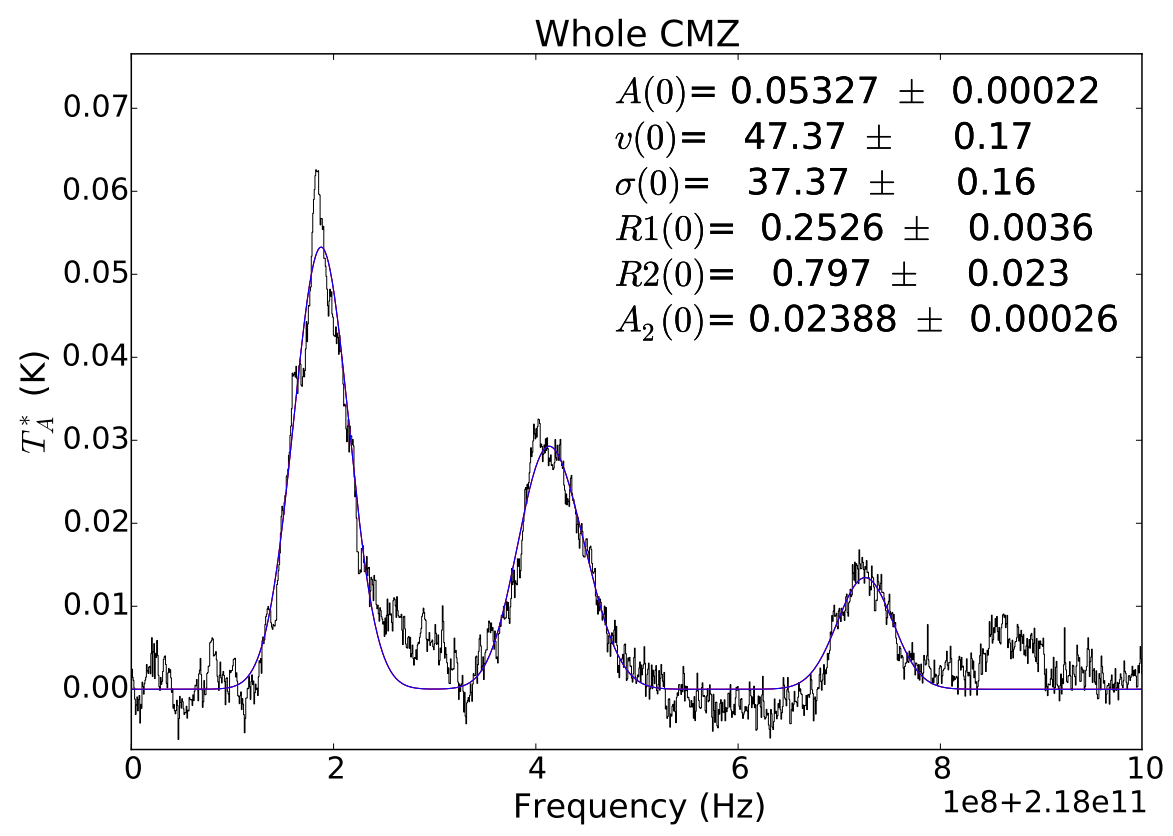

Fig. 17. Spectrum of the whole survey averaged over all pixels. The fit parameters, along with the nominal errors on the parameters, are shown in the legend. A single-component fit was used, though many subtler individual components are evident. Assuming a constant gas density $n=10^{4.5 \pm 0.5} \mathrm{~cm}^{-3}$, the measured ratio implies $T_{\mathrm{G}, \mathrm{CMZ}}=63_{-16}^{+2} \mathrm{~K}$.

M82's inner regions present an interesting contrast to the CMZ. Mühle et al. (2007) report high p- $\mathrm{H}_{2} \mathrm{CO}$-derived temperature $T \sim 200 \mathrm{~K}$, while Weiß et al. (2001) found $\mathrm{NH}_{3}$-based temperature $T \sim 60 \mathrm{~K}$. Taken at face value, these measurements imply that both thermometers show increasing temperatures in starbursts, but they each trace different gas.

\subsection{Implications for star formation}

The star formation properties of the CMZ appear to be different than in the rest of the Galaxy (Yusef-Zadeh et al. 2009, 2010; Immer et al. 2012; Longmore et al. 2013a). It generally appears deficient in star formation tracers relative to its dense gas mass. Kruijssen et al. (2014) and Krumholz \& Kruijssen (2015) suggested that one of the main driving factors in this low star formation rate is an increased threshold for gravitational collapse caused by turbulent pressure. Rathborne et al. (2014) support this claim with observations that the gas column probability distribution function in The Brick is consistent with a purely turbulent origin.

Our observations further confirm that turbulence is an energetically dominant process in the CMZ. We provide an important constraint on the isothermal sound speed, $c_{\mathrm{s}}=0.5-0.9 \mathrm{~km} \mathrm{~s}^{-1}$, which is important for setting the Mach number that is an essential ingredient in turbulence calculations. While the high $c_{\mathrm{s}}$ implies a lower Mach number, the observed line widths more than make up for it; CMZ gas is still more turbulent than Galactic disk gas.

The high temperature also means that the thermal Jeans mass is about a factor of 5 higher than in local clouds (assuming $T_{\mathrm{G}, \mathrm{CMZ}}=60 \mathrm{~K}, T_{\mathrm{G}, \text { local }}=20 \mathrm{~K}, M_{\mathrm{J}, \mathrm{CMZ}}\left(n=10^{5} \mathrm{~cm}^{-3}\right) \approx$ $\left.9 M_{\odot}, M_{\mathrm{J}, \text { local }}\left(n=10^{5} \mathrm{~cm}^{-3}\right) \approx 2 M_{\odot}\right)$; naively this implies a larger typical fragmentation scale and perhaps a preference toward forming high mass cores (Larson 2005), though the higher density in CMZ clouds should balance this. However, many modern theories of star formation now invoke turbulence to provide the core fragmentation spectrum, so the thermal Jeans scale is less relevant for setting core masses (Krumholz \& McKee 2005; Hennebelle \& Chabrier 2011; Padoan \& Nordlund 2011; Federrath \& Klessen 2012; Hennebelle \& Chabrier 2013; Hopkins 2013). The high temperatures and high Jeans mass provide an interesting opportunity to demonstrate that turbulent fragmentation, which occurs at the sonic scale, rather than Jeans fragmentation is responsible for setting the peak position of the core mass function. If turbulence is responsible for setting the core fragmentation scale (Hopkins 2013), it should be possible to find sub-Jeans-mass fragments, and in the warm CMZ it should be easier than in cold, less turbulent local clouds to distinguish the Jeans scale from the sonic scale.

The Equation of State (EOS) in many simulations of starforming regions is generally assumed to follow a fixed form that matches observations of gas in the solar neighborhood (Jappsen et al. 2005; Bonnell et al. 2006; Dale et al. 2012). They assume that $T_{\mathrm{G}}=T_{\mathrm{D}}$ at $n \sim 10^{5} \mathrm{~cm}^{-3}$. Our measurements suggest that gas at this density is still uncoupled from the dust, such that the first "inflection point" in the EOS should be at a higher characteristic density, $n_{\mathrm{c}} \gtrsim 10^{6} \mathrm{~cm}^{-3}$. Future simulations of CMZ-like environments should account for this difference.

\section{Conclusion}

We present the largest gas temperature map of the CMZ to date. We release the data ${ }^{24}$ and the source code ${ }^{25}$ for the entire project.

The main old result from this paper is that the gas temperature in the galactic center molecular clouds is relatively high $(50-120 \mathrm{~K})$ and apparently fairly uniform. The temperatures which we derive are higher than the dust temperature (Güsten et al. 1981, see their conclusion). We have added new information on the large scale of this uniformity and on the association of these temperatures with particular physical mechanisms, but overall we confirm those early results. There is

\footnotetext{
${ }^{24}$ http://thedata.harvard.edu/dvn/dv/APEX-CMZ-1mm

25 https://github.com/adamginsburg/APEX_CMZ_H2CO
} 
warm $\left(T_{\mathrm{G}} \gtrsim 60 \mathrm{~K}\right)$ dense gas pervading the CMZ out to a radius $\sim 200 \mathrm{pc}$.

We have examined the thermal balance in CMZ clouds. The high but variable gas temperatures throughout the CMZ suggest that turbulent heating is the dominant heating mechanism in Galactic Center dense gas. Cosmic rays are not dominant, but may be important in less turbulent sub-regions within the CMZ. We are able to place an upper limit $\zeta_{\mathrm{CR}}<10^{-14} \mathrm{~s}^{-1}$ in the dense gas because a higher CRIR would result in a higher floor temperature than we observe. Dust is a coolant in CMZ dense gas, and no other heating mechanisms should be able to affect the centers of dense clouds.

There are many regions with expected temperatures based on heating from turbulence substantially higher than observed. These regions may have chemistry significantly different from that in local clouds, with more line coolants (e.g., atomic oxygen) available due to the high gas temperature. While we did not investigate the chemistry of CMZ clouds in detail, we suggest that exploring chemistry in $\sim 60-100 \mathrm{~K}, n \gtrsim 10^{4} \mathrm{~cm}^{-3}$ material will be essential for understanding observations of molecular lines in the $\mathrm{CMZ}$ and in other galaxies with similar conditions.

The $\mathrm{H}_{2} \mathrm{CO}$ temperatures are uniformly higher than $\mathrm{NH}_{3}$ $(1-1) /(2-2)$ temperatures but similar to temperatures derived from higher $\mathrm{NH}_{3}$ transitions. The lower temperatures observed in the lower critical density tracer imply that there is a cool, lowdensity component of the molecular gas. This perplexing result is contrary to expectations that the dense gas should be the coldest and deserves further study.

Our new observations are broadly consistent with the orbital model of the CMZ dense gas described by Kruijssen et al. (2015). While there are some individual discrepancies, it at least appears that the near-side clouds (The Brick and the lettered clouds on the path to Sgr B2) have consistent thermal properties. There is a hint that the dust ridge clouds fall along a stream of progressively increasing temperature as a function of age along the predicted orbits. However, the observational uncertainties are also consistent with a constant temperature along the ridge, so these data cannot be taken as direct proof of the orbital model.

Acknowledgements. We thank the staff and observers at APEX for carrying out the service-mode observations. We are grateful to Arnaud Belloche, Axel Weiß, and Carlos de Breuck for assistance in developing the observing strategy, and Per Bergman for his assistance in understanding the SHFI-1 baseline issues. We thank Katharine Johnston for providing the SMA p- $\mathrm{H}_{2} \mathrm{CO} 3_{2,1}-2_{2,0}$ data cube for The Brick. We thank Erik Rosolowsky for advice concerning treatment of the biases inherent in clump extraction, Padelis Papadopoulos for providing commentary on a draft of the paper, Neale Gibson for discussions about parameter constraints, and the referee for a helpful review. T.P. acknowledges support from the Deutsche Forschungsgemeinschaft (DFG) via the SPP (priority program) 1573 "Physics of the ISM". This research was supported by the DFG cluster of excellence "Origin and Structure of the Universe" (JED).

\section{Code bibliography:}

- sdpy https://github.com/adamginsburg/sdpy

- FITS_tools https://github.com/keflavich/FITS_ tools

- aplpy http://aplpy.github.io

- pyradex https://github.com/adamginsburg/pyradex

- myRadex https://github.com/fjdu/myRadex

- pyspeckit http://pyspeckit.bitbucket.org (Ginsburg \& Mirocha 2011)

- astropy http://www.astropy.org

(Astropy

Collaboration et al. 2013)

- wcsaxes http://wcsaxes.readthedocs.org

- spectral cube http://spectral-cube.readthedocs. org
- pvextractor http://pvextractor.readthedocs.org/

- ipython http://ipython. org/ (Pérez \& Granger 2007)

- DESPOTIC https://sites.google.com/a/ucsc.edu/ krumholz/codes/despotic (Krumholz 2014).

\section{References}

Aguirre, J. E., Ginsburg, A. G., Dunham, M. K., et al. 2011, ApJS, 192, 4 Ao, Y. Henkel, C., Menten, K. M., et al. 2013, A\&A, 550, A135

Astropy Collaboration, Robitaille, T. P., Tollerud, E. J., et al. 2013, A\&A, 558, A33

Bally, J. Aguirre, J., Battersby, C., et al. 2010, ApJ, 721, 137

Battersby, C., Bally, J., Ginsburg, A., et al. 2011, A\&A, 535, A128

Battersby, C., Bally, J., Dunham, M., et al. 2014, ApJ, 786, 116

Bayet, E., Yates, J., \& Viti, S. 2011, ApJ, 728, 114

Belloche, A., Müller, H. S. P., Menten, K. M., Schilke, P., \& Comito, C. 2013 A\&A, 559, A47

Bonnell, I. A., Clarke, C. J., \& Bate, M. R. 2006, MNRAS, 368, 1296

Carey, S. J., Clark, F. O., Egan, M. P., et al. 1998, ApJ, 508, 721

Clark, P. C., Glover, S. C. O., Ragan, S. E., Shetty, R., \& Klessen, R. S. 2013, ApJ, 768, L34

Dale, J. E., Ercolano, B., \& Bonnell, I. A. 2012, MNRAS, 427, 2852

Dame, T. M. 2011, arXiv e-prints [arXiv: 1101.1499]

de Vicente, P., Martin-Pintado, J., \& Wilson, T. L. 1997, A\&A, 320, 957

Dunham, M. K. Rosolowsky, E., Evans, N. J., II, et al. 2010, ApJ, 717, 1157

Etxaluze, M. Goicoechea, J. R., Cernicharo, J., et al. 2013, A\&A, 556, A137

Falgarone, E., \& Puget, J.-L. 1995, A\&A, 293, 840

Federrath, C., \& Klessen, R. S. 2012, ApJ, 761, 156

Flower, D. R., Le Bourlot, J., Pineau des Forêts, G., \& Roueff, E. 2000, MNRAS, 314,753

Ghez, A. M. Salim, S., Weinberg, N. N., et al. 2008, ApJ, 689, 1044

Gillessen, S., Eisenhauer, F., Fritz, T. K., et al. 2009, ApJ, 707, L114

Gillessen, S., Eisenhauer, F., Fritz, T. K., et al. 2013, in IAU Symp. 289, ed. R. de Grijs, 2

Ginsburg, A., \& Mirocha, J. 2011, Astrophysics Source Code Library [record ascl:1109.001]

Ginsburg, A. Glenn, J., Rosolowsky, E., et al. 2013, ApJS, 208, 14

Goicoechea, J. R., Etxaluze, M., Cernicharo, J., et al. 2013, ApJ, 769, L13

Goldsmith, P. F. 2001, ApJ, 557, 736

Goto, M., Usuda, T., Geballe, T. R., et al. 2011, PASJ, 63, L13

Goto, M., Indriolo, N., Geballe, T. R., \& Usuda, T. 2013, J. Phys. Chem. A, 117 9919

Goto, M., Geballe, T. R., Indriolo, N., et al. 2014, ApJ, 786, 96

Green, S. 1991, ApJS, 76, 979

Güsten, R., Walmsley, C. M., \& Pauls, T. 1981, A\&A, 103, 197

Güsten, R. Booth, R. S., Cesarsky, C., et al. 2006, in SPIE Conf. Ser., 6267, 14

Heiderman, A., Evans, II, N. J., Allen, L. E., Huard, T., \& Heyer, M. 2010, ApJ, 723, 1019

Hennebelle, P., \& Chabrier, G. 2011, ApJ, 743, L29

Hennebelle, P., \& Chabrier, G. 2013, ApJ, 770, 150

Hollenbach, D. J., \& Tielens, A. G. G. M. 1999, Rev. Mod. Phys., 71, 173

Hopkins, P. F. 2013, MNRAS, 430, 1653

Hüttemeister, S., Wilson, T. L., Bania, T. M., \& Martin-Pintado, J. 1993, A\&A, 280, 255

Immer, K., Menten, K. M., Schuller, F., \& Lis, D. C. 2012, A\&A, 548, A120

Jappsen, A.-K., Klessen, R. S., Larson, R. B., Li, Y., \& Mac Low, M.-M. 2005, A\&A, 435, 611

Johnston, K. G., Beuther, H., Linz, H., et al. 2014, A\&A, 568, A56

Juvela, M., Pelkonen, V.-M., White, G. J., et al. 2012, A\&A, 544, A14

Kamenetzky, J., Glenn, J., Rangwala, N., et al. 2012, ApJ, 753, 70

Kamenetzky, J., Rangwala, N., Glenn, J., Maloney, P. R., \& Conley, A. 2014, ApJ, 795, 39

Kauffmann, J., Pillai, T., \& Zhang, Q. 2013, ApJ, 765, L35

Kennicutt, Jr., R. C. 1998, ApJ, 498, 541

Kennicutt, R. C., \& Evans, N. J. 2012, ARA\&A, 50, 531

Klein, B., Hochgürtel, S., Krämer, I., et al. 2012, A\&A, 542, L3

Kruijssen, J. M. D., \& Longmore, S. N. 2013, MNRAS, 435, 2598

Kruijssen, J. M. D., Longmore, S. N., Elmegreen, B. G., et al. 2014, MNRAS, 440,3370

Kruijssen, J. M. D., Dale, J. E., \& Longmore, S. N. 2015, MNRAS, 447, 1059

Krumholz, M. R. 2014, MNRAS, 437, 1662

Krumholz, M. R., \& Kruijssen, J. M. D. 2015, MNRAS, 453, 739

Krumholz, M. R., \& McKee, C. F. 2005, ApJ, 630, 250

Larson, R. B. 2005, MNRAS, 359, 211

Le Bourlot, J., Pineau des Forêts, G., \& Flower, D. R. 1999, MNRAS, 305, 802 


\section{A. Ginsburg et al.: APEX CMZ $\mathrm{H}_{2} \mathrm{CO}$}

Leroy, A. K., Walter, F., Sandstrom, K., et al. 2013, AJ, 146, L19

Lis, D. C., \& Menten, K. M. 1998, ApJ, 507, 794

Lis, D. C., Carlstrom, J. E., \& Keene, J. 1991, ApJ, 380, 429

Lis, D. C., Li, Y., Dowell, C. D., \& Menten, K. M. 1999, in The Universe as Seen by ISO, eds. P. Cox, \& M. Kessler, ESA, 427, 627

Lis, D. C., Schilke, P., Bergin, E. A., et al. 2014, ApJ, 785, 9

Loenen, A. F., Spaans, M., Baan, W. A., \& Meijerink, R. 2008, A\&A, 488, L5

Longmore, S. N., Rathborne, J., Bastian, N., et al. 2012, ApJ, 746, 117

Longmore, S. N., Bally, J., Testi, L., et al. 2013a, MNRAS, 429, 987

Longmore, S. N., Bally, J., Testi, L., et al. 2013b, MNRAS, 433, L15

Mangum, J. G., \& Wootten, A. 1993, ApJS, 89, 123

Mangum, J. G., Emerson, D. T., \& Greisen, E. W. 2007, A\&A, 474, 679

Mangum, J. G., Darling, J., Henkel, C., \& Menten, K. M. 2013, ApJ, 766, 108

Mauersberger, R., Henkel, C., Wilson, T. L., \& Walmsley, C. M. 1986, A\&A, 162,199

Meijerink, R., Spaans, M., Loenen, A. F., \& van der Werf, P. P. 2011, A\&A, 525, A119

Menten, K. M., Wilson, R. W., Leurini, S., \& Schilke, P. 2009, ApJ, 692, 47

Mills, E. A. C., \& Morris, M. R. 2013, ApJ, 772, 105

Molinari, S. S. Bally, J., Noriega-Crespo, A., et al. 2010, A\&A, 518, L100

Molinari, S., Bally, J., Noriega-Crespo, A., et al. 2011, ApJ, 735, L33

Morris, M., \& Serabyn, E. 1996, ARA\&A, 34, 645

Morris, M., Zuckerman, B., Palmer, P., \& Turner, B. E. 1973, ApJ, 186, 501

Mühle, S., Seaquist, E. R., \& Henkel, C. 2007, ApJ, 671, 1579

Mundy, L. G., Evans, II, N. J., Snell, R. L., \& Goldsmith, P. F. 1987, ApJ, 318, 392

Nelson, R. P., \& Langer, W. D. 1999, ApJ, 524, 923

Neufeld, D. A., \& Dalgarno, A. 1989, ApJ, 340, 869

Nummelin, A., Bergman, P., Hjalmarson, A., et al. 1998, ApJS, 117, 427

Ott, J., Weiss, A., Staveley-Smith, L., Henkel, C., \& Meier, D. S. 2014, ApJ, 785,16

Padoan, P., \& Nordlund, Å. 2011, ApJ, 730, 40

Papadopoulos, P. P. 2010, ApJ, 720, 226

Papadopoulos, P. P., \& Thi, W.-F. 2013, in Cosmic Rays in Star-Forming Environments, eds. D. F. Torres, \& O. Reimer, Astrophys. Space Sci. Proc., 34,41
Papadopoulos, P. P., Thi, W.-F., Miniati, F., \& Viti, S. 2011, MNRAS, 414, 1705 Pérez, F., \& Granger, B. E. 2007, Comput. Sci. Engineering, 9, 21

Pillai, T., Wyrowski, F., Carey, S. J., \& Menten, K. M. 2006, A\&A, 450, 569

Pineda, J. E., Goodman, A. A., Arce, H. G., et al. 2010, ApJ, 712, L116

Rathborne, J. M. Longmore, S. N., Jackson, J. M., et al. 2014, ApJ, 795, L25

Reid, M. J., Menten, K. M., Zheng, X. W., Brunthaler, A., \& Xu, Y. 2009, ApJ, 705,1548

Riquelme, D., Amo-Baladrón, M. A., Martín-Pintado, J., et al. 2013, A\&A, 549, A36

Rodriguez-Fernandez, N. J., Combes, F., Martin-Pintado, J., Wilson, T. L., \& Apponi, A. 2006, A\&A, 455, 963

Rosolowsky, E., \& Blitz, L. 2005, ApJ, 623, 826

Rosolowsky, E., \& Leroy, A. 2006, PASP, 118, 590

Rosolowsky, E. W., Pineda, J. E., Kauffmann, J., \& Goodman, A. A. 2008, ApJ, 679, 1338

Schöier, F. L., van der Tak, F. F. S., van Dishoeck, E. F., \& Black, J. H. 2005 A\&A, 432, 369

Shetty, R., Beaumont, C. N., Burton, M. G., Kelly, B. C., \& Klessen, R. S. 2012, MNRAS, 425, 720

Shirley, Y. L. 2015, PASP, 127, 299

Sofue, Y. 1995, PASJ, 47, 527

Traficante, A. Calzoletti, L., Veneziani, M., et al. 2011, MNRAS, 416, 2932

van der Tak, F. F. S., Black, J. H., Schöier, F. L., Jansen, D. J., \& van Dishoeck, E. F. 2007, A\&A, 468, 627

van der Werf, P. P. Isaak, K. G., Meijerink, R., et al. 2010, A\&A, 518, L42

Vassilev, V., Meledin, D., Lapkin, I., et al. 2008, A\&A, 490, 1157

Walsh, A. J. Breen, S. L., Britton, T., et al. 2011, MNRAS, 416, 1764

Weiß, A., Neininger, N., Henkel, C., Stutzki, J., \& Klein, U. 2001, ApJ, 554, L143

Wiesenfeld, L., \& Faure, A. 2013, MNRAS, 432, 2573

Wolniewicz, L., Simbotin, I., \& Dalgarno, A. 1998, ApJS, 115, 293

Wootten, A., Evans, II, N. J., Snell, R., \& vanden Bout, P. 1978, ApJ, 225, L143

Wrathmall, S. A., Gusdorf, A., \& Flower, D. R. 2007, MNRAS, 382, 133

Yusef-Zadeh, F., Hewitt, J. W., Arendt, R. G., et al. 2009, ApJ, 702, 178

Yusef-Zadeh, F., Lacy, J. H., Wardle, M., et al. 2010, ApJ, 725, 1429

Yusef-Zadeh, F., Hewitt, J. W., Arendt, R. G., et al. 2013, ApJ, 762, 33 


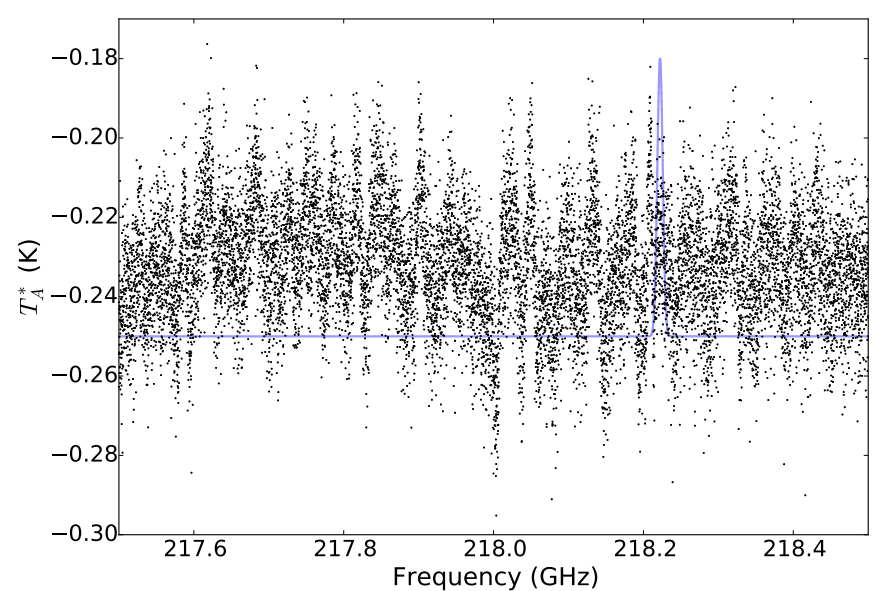

Fig. A.1. An example showing some of the worst baselines observed. The plotted spectrum is from an observation on April 2, 2014, showing the average of the $5 \%$ worst spectra. The blue curve shows a $\sigma=5 \mathrm{~km} \mathrm{~s}^{-1}\left(F W H M=11.75 \mathrm{~km} \mathrm{~s}^{-1}\right)$ line centered at the $0 \mathrm{~km} \mathrm{~s}^{-1}$ position of $\mathrm{p}-\mathrm{H}_{2} \mathrm{CO} 3_{0,3}-2_{0,2}$, illustrating that the baseline "ripples" have widths comparable to the observed lines. While we selected the worst $5 \%$ in this case, nearly all spectra are affected by these sorts of baselines, and the shape and amplitude varies dramatically and unpredictably. The variation, unpredictable though it is, works in our favor as it averages out over multiple independent observations. The $218 \mathrm{GHz}$ region shown here is also the worst-affected; the $220 \mathrm{GHz}$ range that includes the ${ }^{13} \mathrm{CO}$ lines generally exhibits smoother and lower-amplitude baseline spectra.

\section{Appendix A: Baseline removal}

The baselines in our APEX-1 (SHFI) data were particularly problematic, more than is usual in modern heterodyne obervations. The $218 \mathrm{GHz}$ window we have observed is particularly sensitive to resonances within the APEX-1 receiver that vary on $<1$ min timescales; our off-position calibrations were performed about once per minute and therefore were not rapid enough to mitigate this problem completely. The baselines can broadly be described as smoothly varying ripples on the scale of $\sim 1 / 10$ the spectral window, plus more rapidly varying ripples on $20-40 \mathrm{~km} \mathrm{~s}^{-1}$ scales. In principle, this is a straightforward problem of identifying the fourier components associated with each of these scales and subtracting them.
In practice, we discovered that it was not possible to remove the dominant baseline structure on either scale without significantly affecting the underlying spectral data. We simulated a variety of fourier-space suppression approaches by adding synthetic signal to baseline spectra extracted from the first few principle component analysis (PCA) components of the real spectra. The PCA extraction approach is able to pull out the dominant baseline components very effectively, but it inevitably includes significant signal in the top few most correlated components, especially for the strong $\mathrm{H}_{2} \mathrm{CO}$ and ${ }^{13} \mathrm{CO}$ lines. We therefore abandoned it for the final data reduction.

For reference, we show an example spectrum that we believe to consist entirely of baseline ripples in Fig. A.1.

\section{Appendix B: Spectral fits for regions and apertures}

We provide a catalog of line ratios measured from apertureextracted spectra along with figures showing the best fit model for each spectrum. We have extracted spectra in regions with significant $\mathrm{p}-\mathrm{H}_{2} \mathrm{CO}$ emission and in commonly studied individual regions. This catalog is intended to provide archival value from our data set and enable future studies of individual regions.

In the hand-extracted source table, three types of region are included:

1. Circular regions-of-interest (Fig. B.1a);

2. Rectangular regions-of-interest (Fig. B.1b);

3. $8^{\prime} \times 8^{\prime}$ square observation fields (Fig. B.1c).

The fit tables include multicomponent spectral fits with and without spline-based baseline removal (see Sect. 4.2). Spatial parameters for the box (GLON, GLAT, boxheight, and boxwidth) or circle (GLON, GLAT, and radius) regions are included. The average dust column and dust temperature are reported (higaldusttem and higalcolumndens). The best fit (maximum likelihood) parameters for gas temperature, $\mathrm{p}-\mathrm{H}_{2} \mathrm{CO}$ column, and $\mathrm{H}_{2}$ density are included along with their $\pm 1 \sigma$ marginalized limits. The full column description is in the tables/README.rst file in the source code repository, along with the tables in IPAC format.

The dendrogram property table is also included in the repository and described in the README. 


\section{A. Ginsburg et al.: APEX CMZ $\mathrm{H}_{2} \mathrm{CO}$}

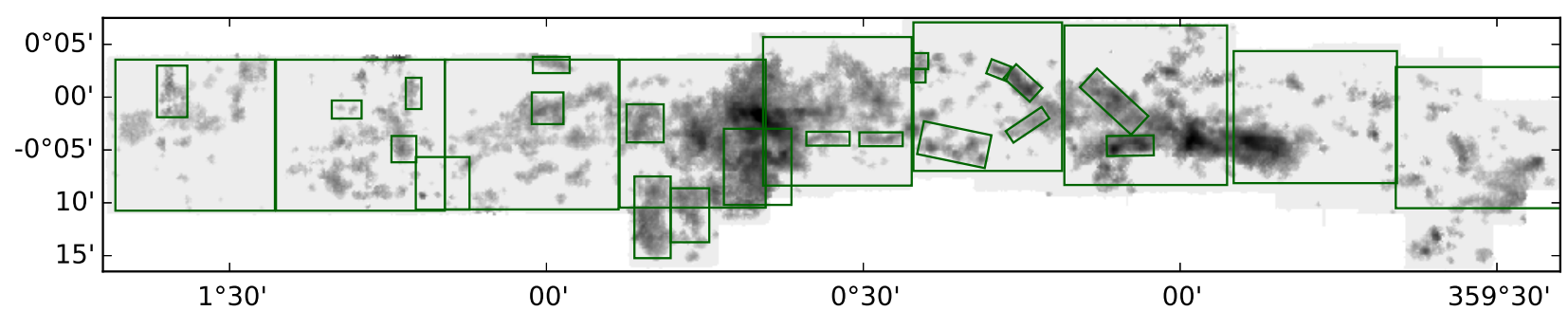

(a)

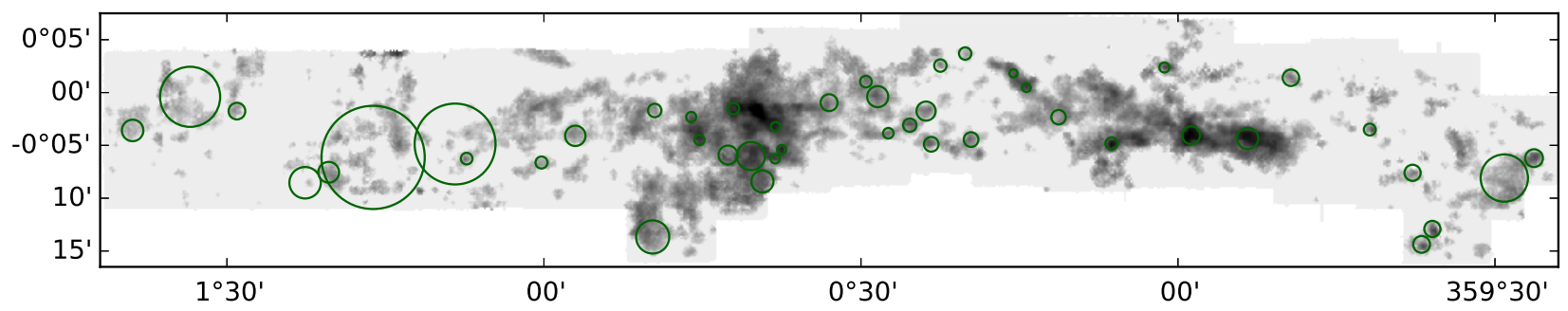

(b)

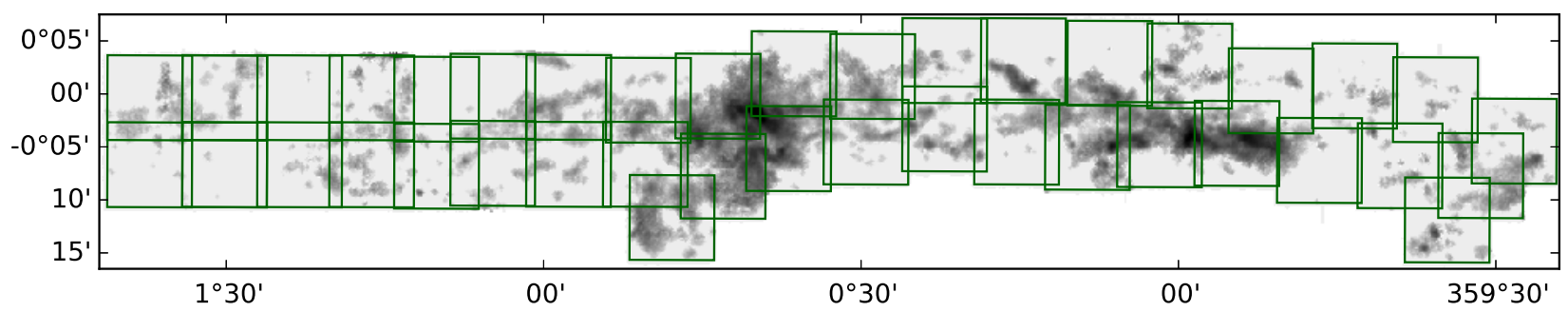

(c)

Fig. B.1. Figures showing the extracted regions. The background is the integrated masked $\mathrm{p}-\mathrm{H}_{2} \mathrm{CO} 3_{0,3}-2_{0,2}$ image. a) Box regions created for large-scale averaging and to track the morphology of individual clumps. b) Circular regions selected to highlight points of interest c) $8^{\prime} \times 8^{\prime}$ Box regions matching the observational setup in 2014. 


\section{Appendix C: Source code and data release}

The reduced and raw data are made available via the CfA dataverse (doi:10.7910/DVN/27601). The reduced data, including cubes, integrated images, and catalogs, are also made available at the CDS.

The source code for this project in its entirety is available at https://github.com/adamginsburg/APEX_CMZ_ $\mathrm{H} 2 \mathrm{CO}$. Because the archives are public, we include scripts to download and process the raw data so that all steps of the analysis performed here can be performed by any individual with access to a computer. We provide a script download_raw_files.py that will retrieve the raw .apex data from the ESO archive and from the dataverse; it requires a valid ESO archive account to use. The total data reduction process takes $\sim 30 \mathrm{~h}$ on a 48 -core, 2012-era linux machine, though most steps are not parallelized so the process may be faster on more recent machines with fewer cores. About $300 \mathrm{~GB}$ of free space are required to store the raw, intermediate, and reduced data products, though the final products are $<30 \mathrm{~GB}$, with an actual size depending on whether the baseline-subtracted versions are kept separate from the original files. The download process may take substantially longer than the data reduction.

To fully reproduce the data products in this paper, some effort is required to set up the appropriate directory structure. The file reduction/run_pipeline_cleese.py provides a useful template for informing the code about the appropriate directory location, and reduction/README.rst includes more detailed instructions. The raw . apex data files need to be stored in a common location, and output directories need to be specified for the merged data files, each epoch's data (one for the Ao et al. 2013, data, one for the 2013 data, and one for the 2014/2015 data), diagnostic plots, and the subcubes for each molecule. The function do_everything in reduction/make_apex_cubes.py will then run the pipeline end-to-end.

\section{Appendix D: A detailed example showing parameter constraints}

In Sect. 4.2, we discussed the fitting approach used to determine a best-fit temperature and uncertainties on that temperature. Here, we go through a detailed example showing projections of the full 3D temperature-density-column parameter space we explored and the constraints each measurement places in each phase space.

Figures D.1-D.3 show the 3D parameter space of the LVG models viewed from its three unique faces. Each figure has 4 panels, each of which shows the effect of one of our measurements in constraining the available parameter space. All panels include the marginalized likelihood contours. Figure D.4 shows slices through the LVG models at the maximum likelihood position. The panels show the predicted line brightness of the $3_{0,3}-2_{0,2}$ and $3_{2,1}-2_{2,0}$ lines in each direction through the LVG parameter cube.

Figure D.5 shows the marginalized one-dimensional distributions for each parameter along with the constraints provided by each measurement. Note that the posterior is not simply the product of the other displayed distributions; for example, very high temperatures require low volume and high column densities that occupy a small fraction of the total integrated likelihood.

In the script file example_parplot_constraints.py in the associated repository, we examine the systematic uncertainties in the fitting process. Using a known density, temperature, and column, we then use the fitting process applied to the dendrogram objects and the hand-extracted regions to derive our measured temperatures. Over a range $10^{4}<n<10^{7} \mathrm{~cm}^{-3}$, the maximum likelihood estimator recovers the true temperature to within $25 \%$ for all temperatures, and to $10 \%$ over the range $10^{4.25}<n<10^{5.5} \mathrm{~cm}^{-3}$. At densities lower than $n<10^{4} \mathrm{~cm}^{-3}$, the recovered temperature is significantly lower than the true temperature, which is expected since we impose a prior that $n>10^{4} \mathrm{~cm}^{-3}$. Curiously, the expectation value is more biased than the maximum likelihood estimator, with errors reaching $30 \%$ and with a much stronger dependence on the input temperature. 

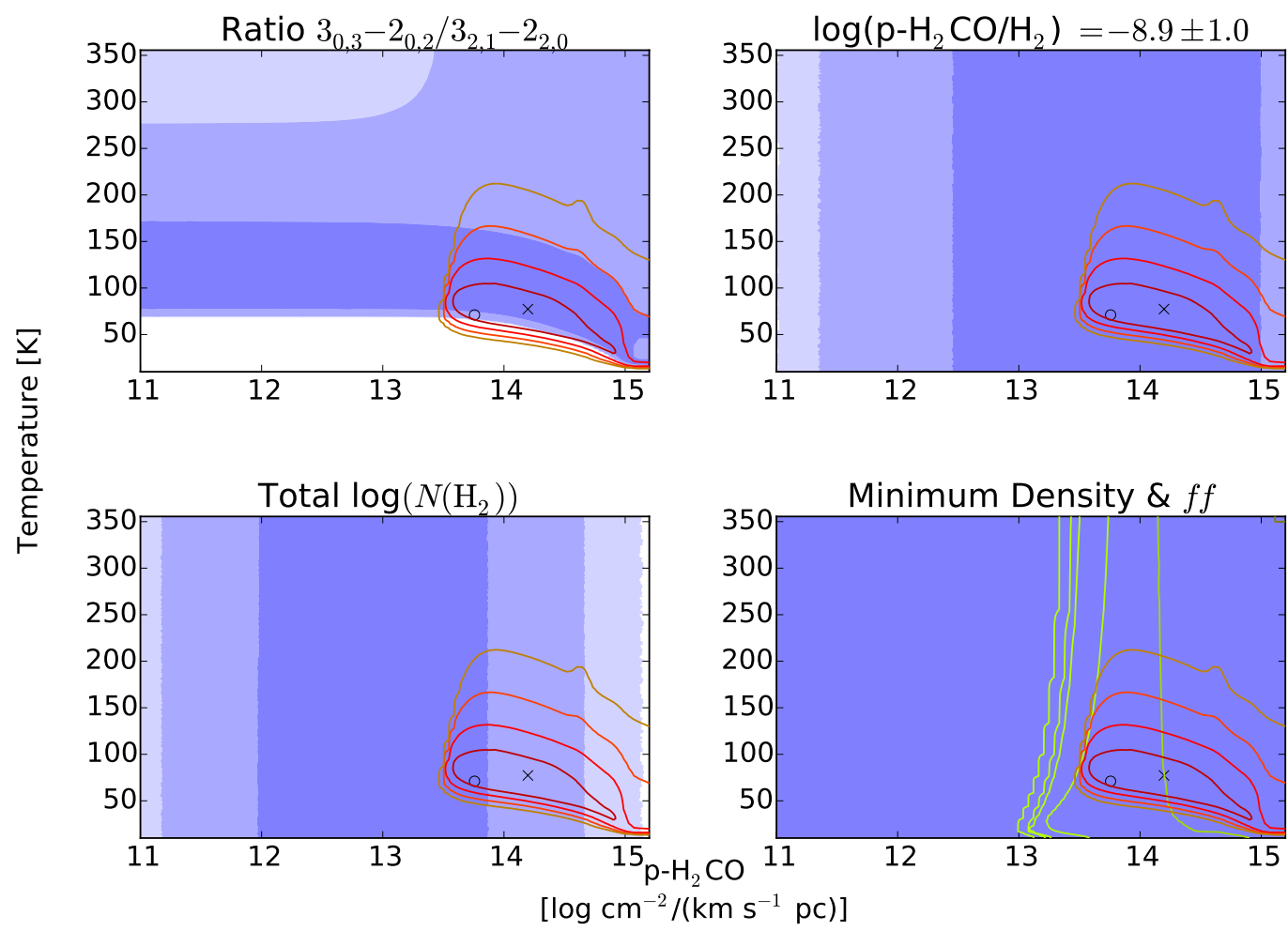

Fig. D.1. The parameter constraints for "cloud c" (Fig. 5) projected (marginalized) onto the temperature/column density plane. (top left) Constraints imposed by the measured ratio $\mathrm{p}-\mathrm{H}_{2} \mathrm{CO}_{v}\left(3_{2,1}-2_{2,0}\right) / S_{v}\left(3_{0,3}-2_{0,2}\right)$ are shown in the background filled colors, with significance ranges progressing from dark-light from $1-3 \sigma$. The red line contours show the joint constraints imposed by including restrictions on the total column density, volume density, filling factor, and abundance, with $1,2,3$, and $4 \sigma$ contours shown. The $\times$ marks the expectation value and the $\circ$ marks the maximum likelihood value. (top right) The same colorscheme as before, showing the constraints imposed by assuming the abundance of $\mathrm{p}-\mathrm{H}_{2} \mathrm{CO}$ relative to $\mathrm{H}_{2}$ is as labeled. The abundance does not constrain these parameters, but in Fig. D.2, the abundance rules out a substantial region of parameter space. (bottom left) The same colorscheme as before, showing the constraints imposed by using the measured mean volume density as a lower limit. In this figure, the volume density imposes no constraint, but in Figs. D.2 and D.3 it is important. (bottom right) The same colorscheme as before, showing the constraints imposed by the measured total column density of $\mathrm{H}_{2}$. The green contours show constraints imposed by the lower limit on the area filling factor.

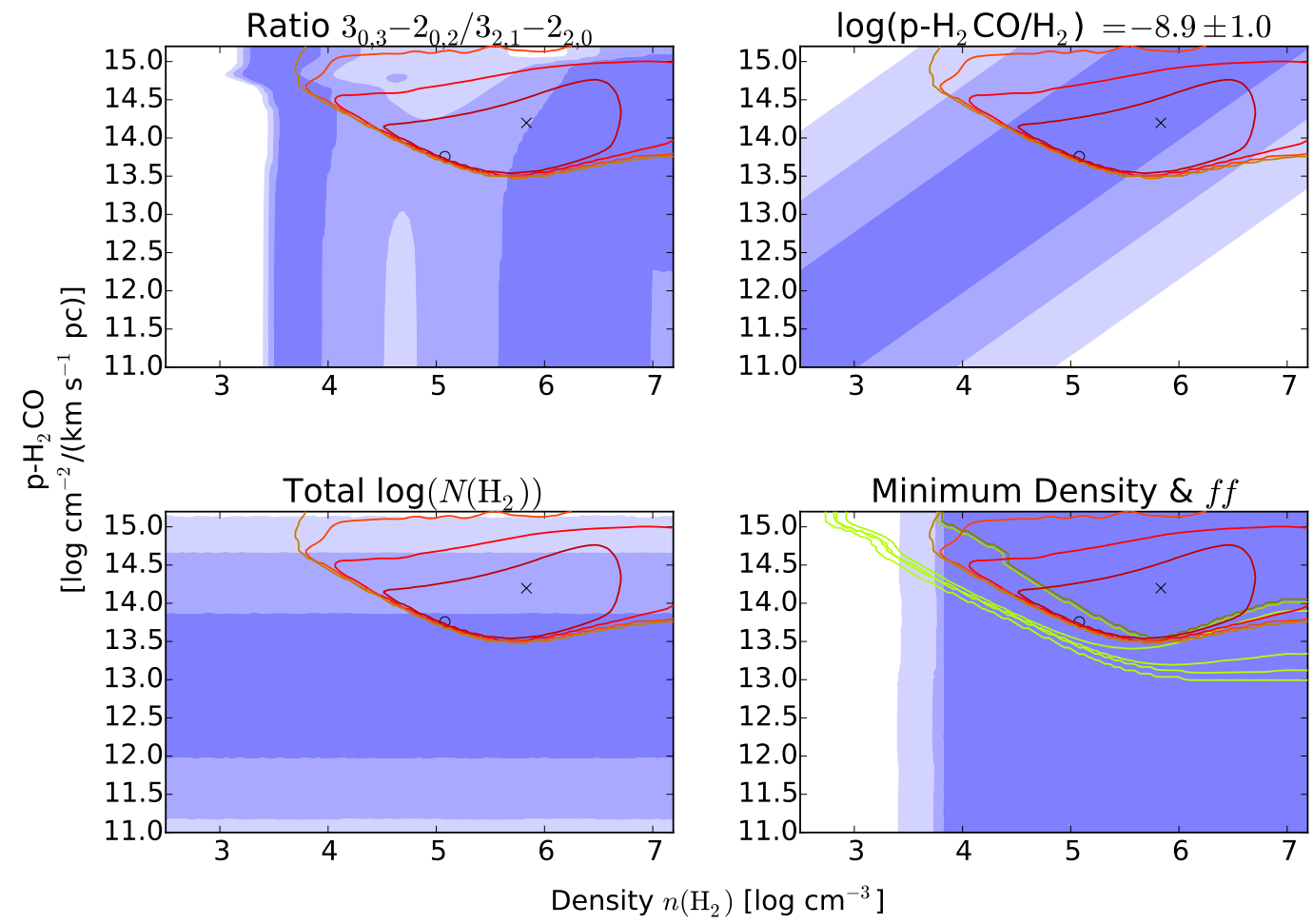

Fig. D.2. The constraints in density-column parameter space. See Fig. D.1 for details. 


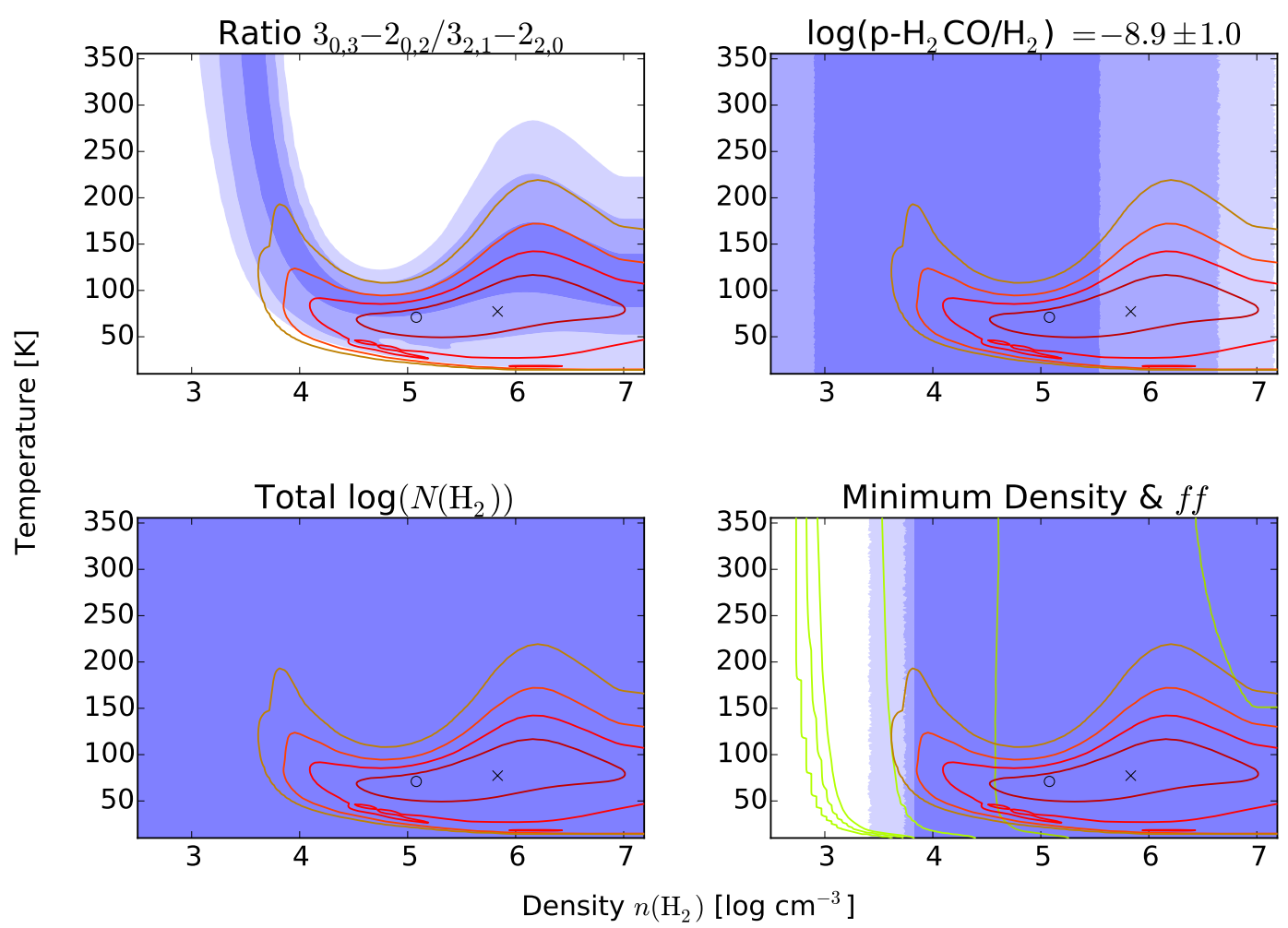

Fig. D.3. The constraints in density-temperature parameter space. See Fig. D.1 for details.

c:G0.38+0.04
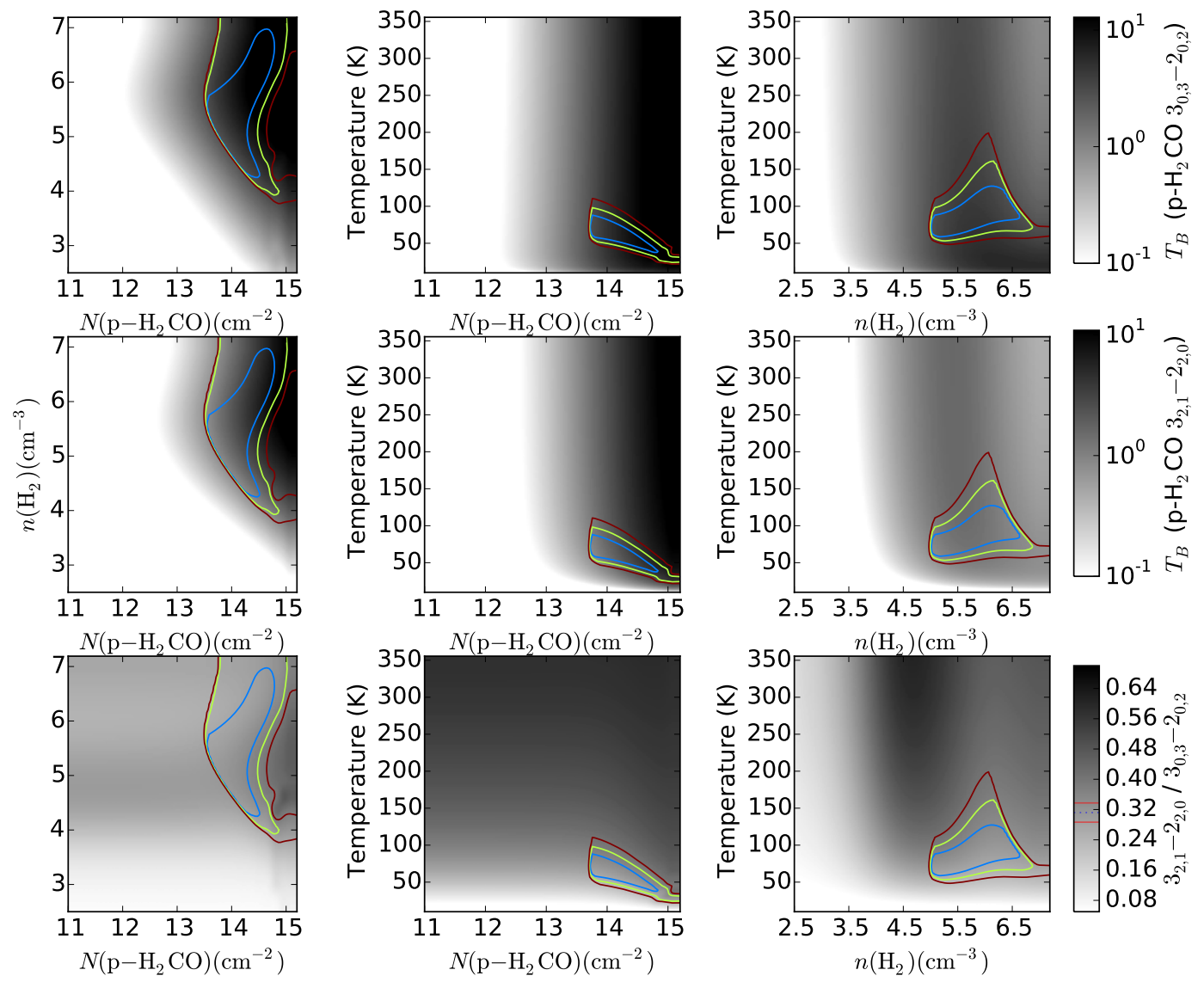

Fig. D.4. The line brightness of $\mathrm{p}-\mathrm{H}_{2} \mathrm{CO} 3_{0,3}-2_{0,2}$ (top row) and $\mathrm{p}-\mathrm{H}_{2} \mathrm{CO} 3_{2,1}-2_{2,0}$ (middle row) and p- $\mathrm{H}_{2} \mathrm{CO} S_{v}\left(3_{2,1}-2_{2,0}\right) / S_{v}\left(3_{0,3}-2_{0,2}\right)($ bottom row) in the three different projections of parameter space. The grayscale images correspond to a slice through the parameter spaces at the location of the best-fit parameter. The colored contours show the allowed marginalized regions in each parameter space as described in the Fig. D.1 caption. 

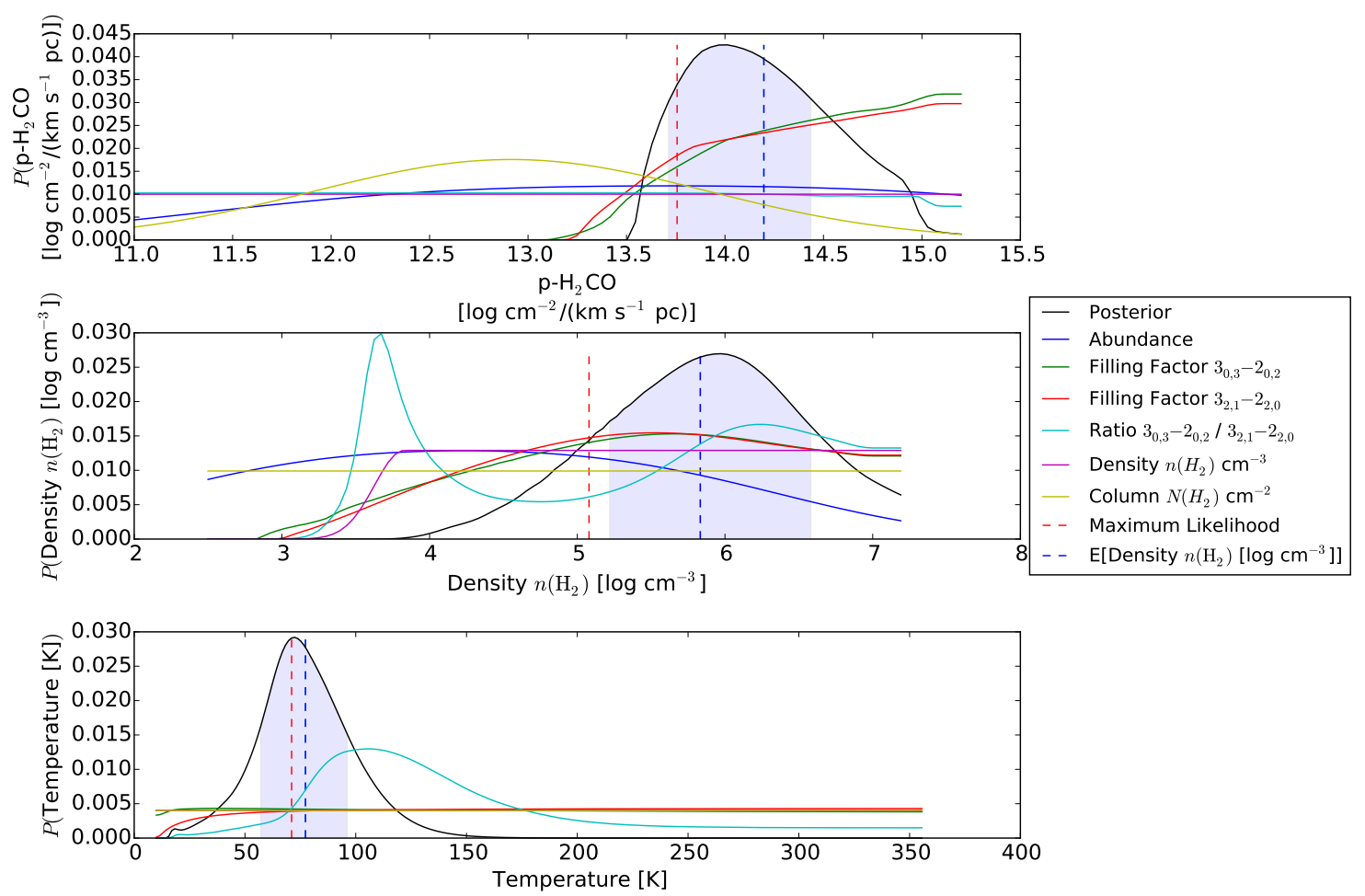

Fig. D.5. The one-dimensional probability distributions for each parameter. The legend describes the lines. The blue shaded area shows the highest $68 \%$ probability region. The maximum-likelihood and expectation value are generally close for temperature but can be very different for column and density.

\section{Appendix E: Smoothed maps}

In many regions, signal was detected over large areas though the peak signal-to-noise ratio remained low. In this Appendix, we display smoothed versions of many of the main figures in the paper to provide a record of features that were weakly detected. The smoothed maps may also give a sense of how the temperature varies with spatial scale.

The cubes were smoothed in the spatial dimensions with a $\sigma_{F W H M}=33.84^{\prime \prime}$ Gaussian to achieve a resolution $45^{\prime \prime}$ and $\sigma_{v, F W H M}=3 \mathrm{~km} \mathrm{~s}^{-1}$ in the spectral direction. The smoothed cubes were also downsampled by a factor of 2 in the spectral direction (the smoothing and downsampling are independent). The same signal extraction analysis described in Sect. 3 was used with a threshold $T_{\mathrm{A}}>3 \sigma$.

Figure E. 3 shows the peak signal-to-noise in both the unsmoothed and the smoothed maps to indicate where the temperature measurements can be relied upon. These figures show the variable-opacity mask used in most of the other figures in this paper, with black corresponding to fully transparent and white fully opaque.

\section{E.1. Dendrogram cubes}

The dendrogram-extracted catalog was used to build a temperature PPV data cube for visualization purposes. Each voxel was assigned a value corresponding to the temperature in the smallest dendrogram structure it was included in. For example, a voxel corresponding to a leaf has the temperature measured for that leaf, while a voxel that is not in a leaf but is part of a structure would be assigned the temperature of the smallest structure in which it is included. The rest of the cube was assigned NAN values. This cube is similar to a smoothed cube, but smoothed over connected structures rather than with a symmetric beam. The cube is interesting for visualization purposes, but should be treated with some skepticism: structures that are connected in PPV space in the $3_{0,3}-2_{0,2}$ line do not generally have the same, constant temperature, but they are forced by construction to have the same temperature in this cube. Despite this caveat, comparison of the dendrogram figures (Fig. E.4) to the directly averaged figures (Figs. E.2 and 7) shows that the dendrogram objects are effectively averages over the included area in most cases. Since there is less noise in these figures, it is easier to identify interesting features in them by eye. 


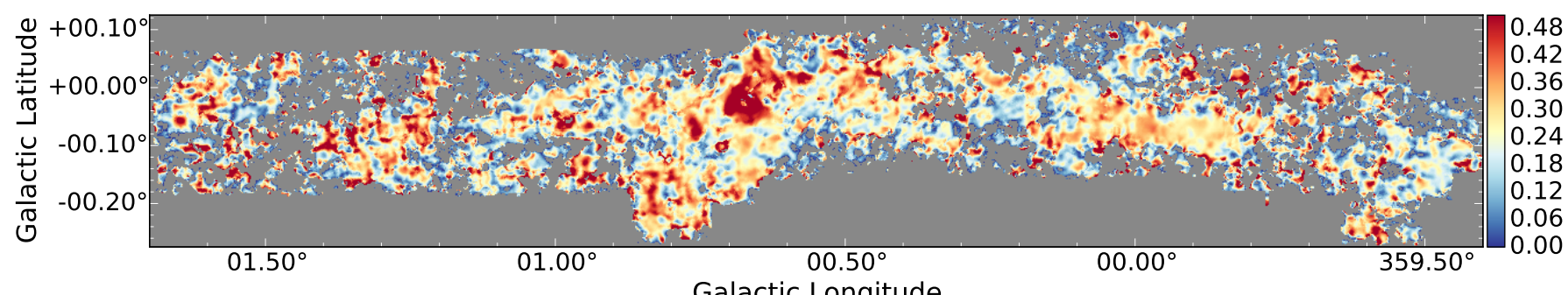

(a)

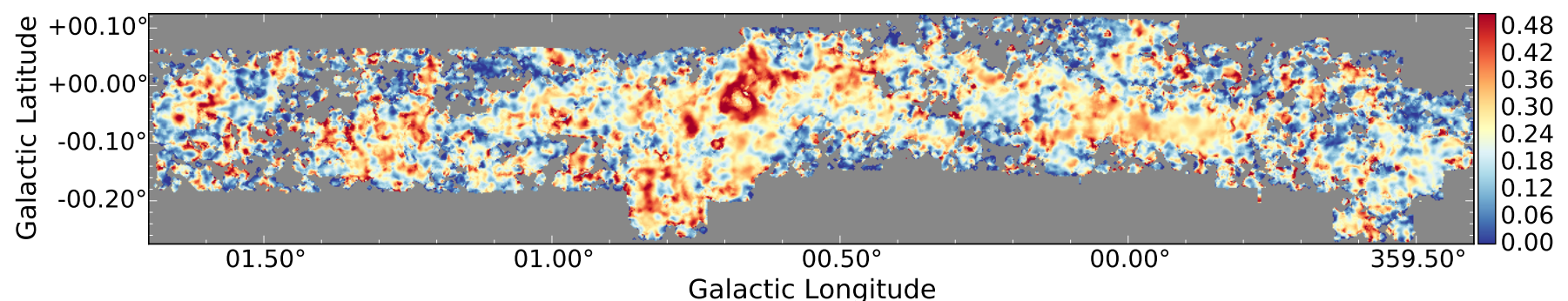

(b)

Fig. E.1. Same as Fig. 3, but smoothed with a 34" FWHM Gaussian.

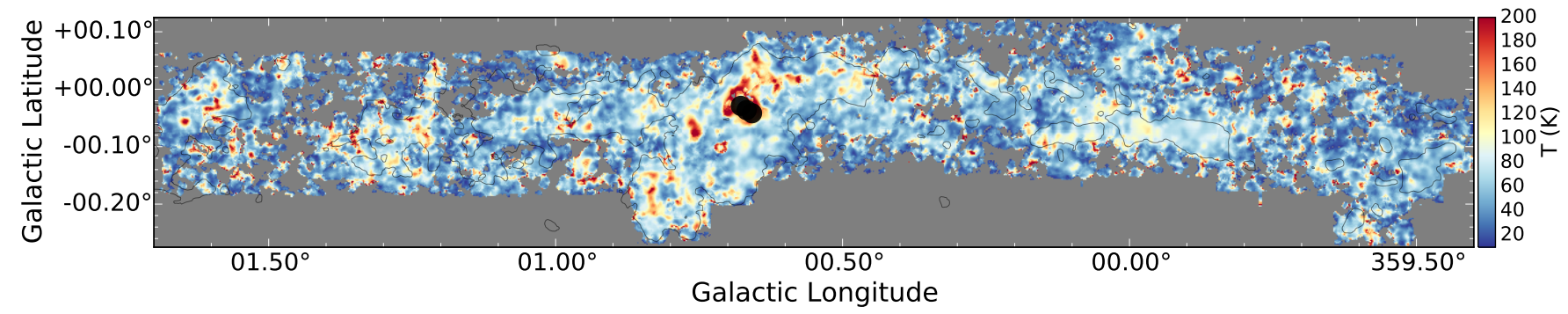

(a)

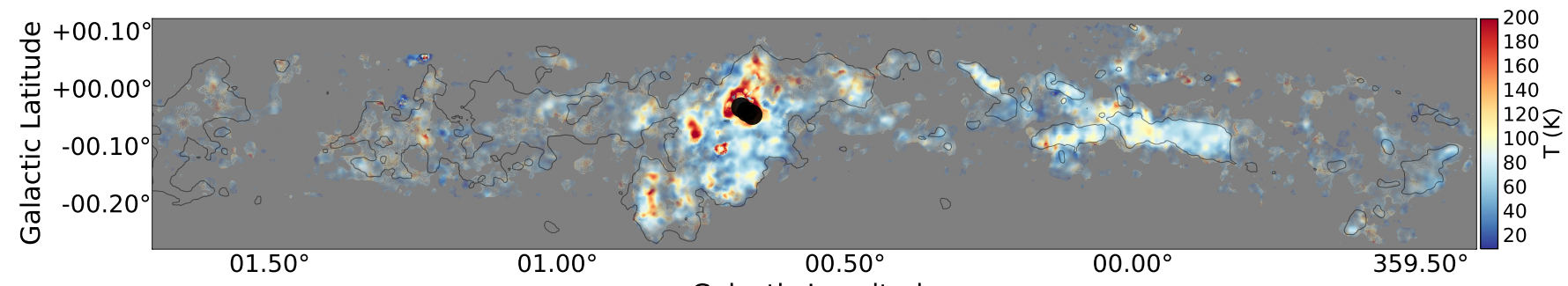

Galactic Longitude

(b)

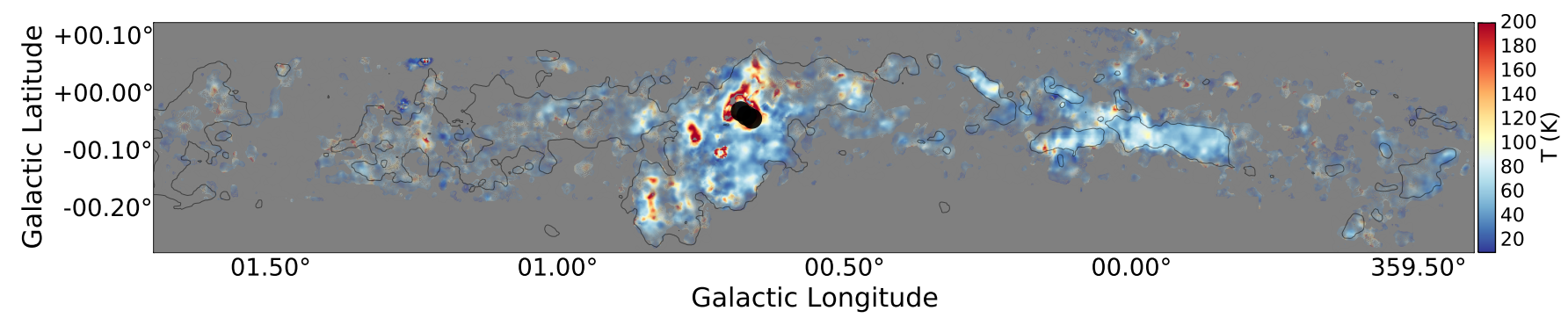

(c)

Fig. E.2. Temperature maps using the smoothed data assuming fixed $X_{\mathrm{p}-\mathrm{H}_{2} \mathrm{CO}}=1.2 \times 10^{-9}$ and $n\left(\mathrm{H}_{2}\right)$. The maps in b) and c) are masked by signal-to-noise as in Fig. 3. The thin black contours are at a column $N\left(\mathrm{H}_{2}\right)=5 \times 10^{22} \mathrm{~cm}^{-2}$ from the Herschel SED fit maps. a) $n\left(\mathrm{H}_{2}\right)=10^{4} \mathrm{~cm}^{-3}$, no mask b) $n\left(\mathrm{H}_{2}\right)=10^{4} \mathrm{~cm}^{-3}$ c) $n\left(\mathrm{H}_{2}\right)=10^{5} \mathrm{~cm}^{-3}$. Figure E.5 shows the same figures, but with varying abundance and fixed $n\left(\mathrm{H}_{2}\right)$. 


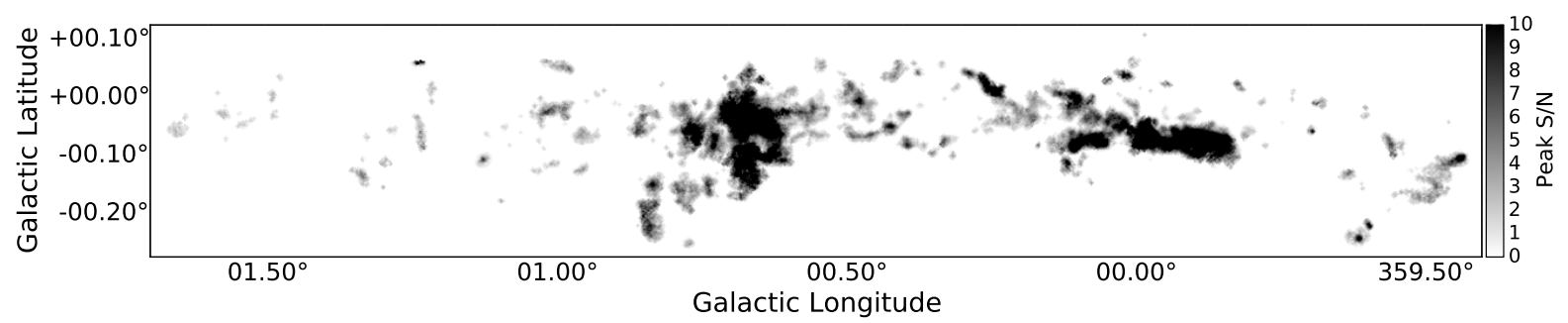

(a)

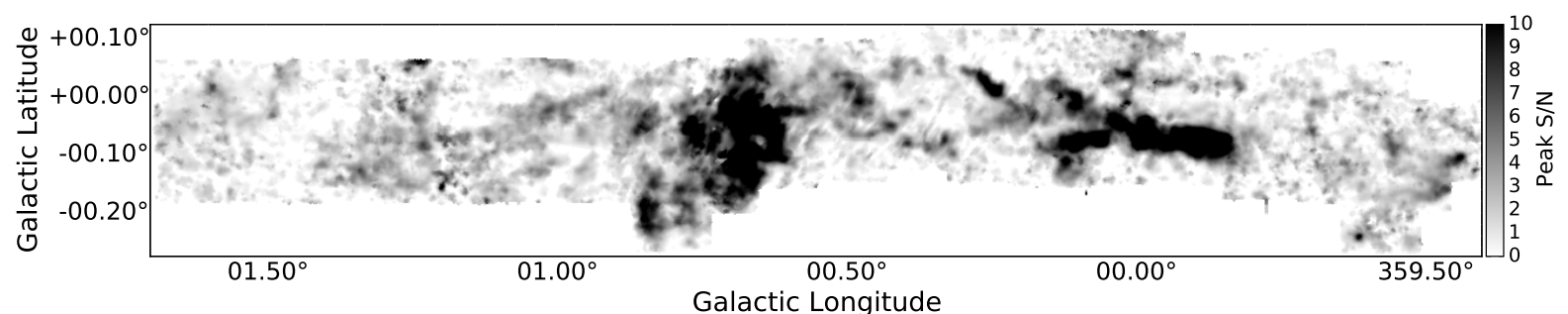

(b)

Fig. E.3. Map of the peak signal-to-noise in the p- $\mathrm{H}_{2} \mathrm{CO} 3_{0,3}-2_{0,2}$ line with no smoothing (top) and with 33.84" smoothing (bottom). These maps give an indication of the reliability of the temperatures extracted in Fig. E.2. The colorbars are intentionally saturated at $S / N>10$ since above this threshold, the temperatures are reliable as long as they are in the $T_{\mathrm{G}}<150 \mathrm{~K}$ regime.

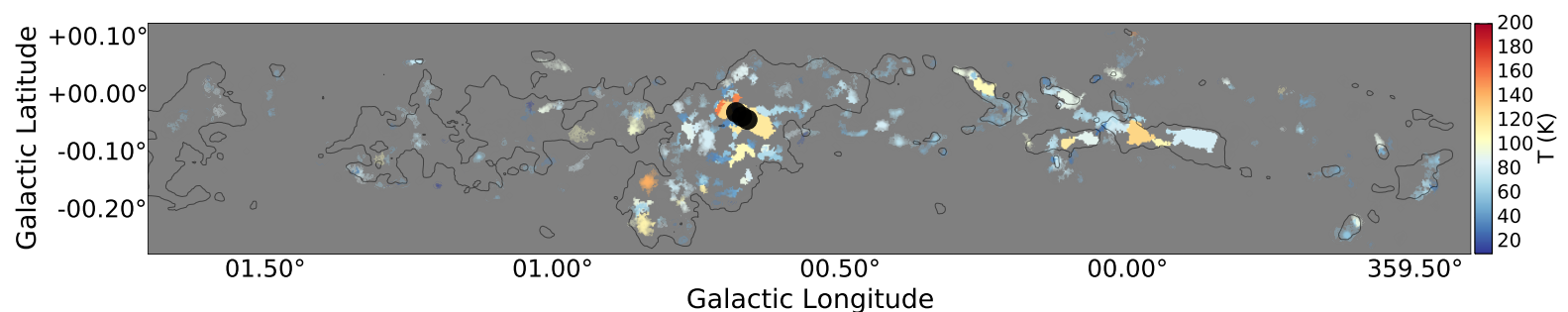

(a)

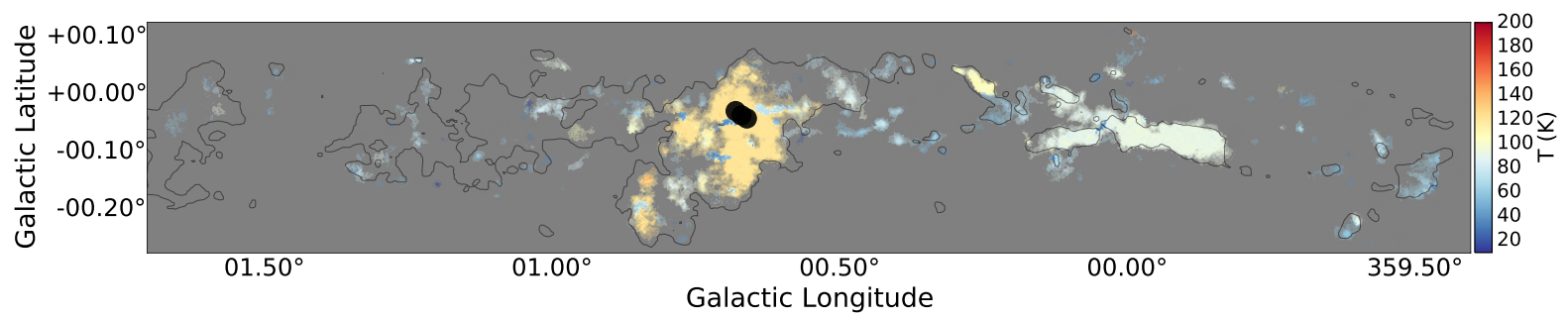

(b)

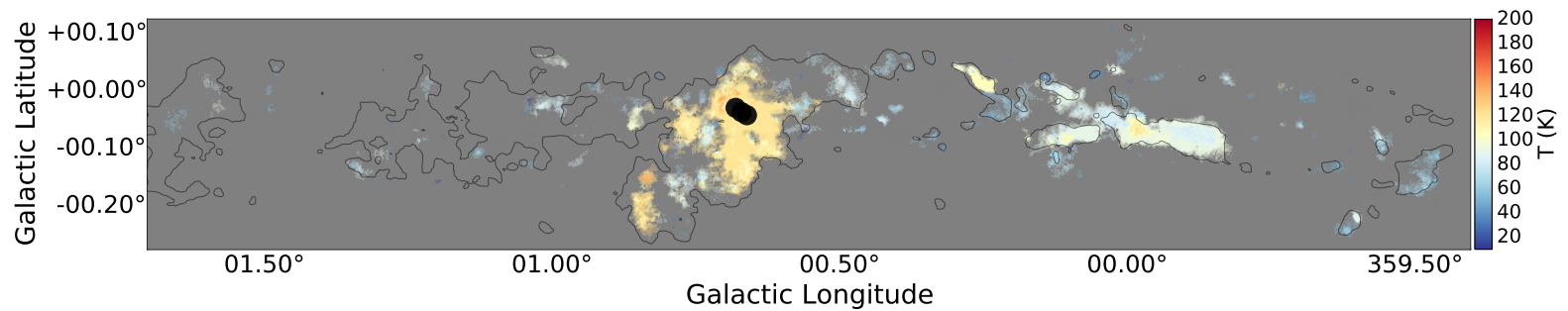

(c)

Fig. E.4. Maps generated by building a data cube in which each voxel has been replaced with the average temperature from the smallest associated dendrogram-extracted clump. These maps can be thought of as adaptively-smoothed maps, where the smoothing kernel is matched to the source size. a) The mean temperature along each line of sight through the dendrogram-extracted cube, where only the leaf nodes have been included b) The same as a), but including all ancestor nodes in addition to the leaf nodes. c) The same as b), but weighted by the $3_{0,3}-2_{0,2}$ brightness. In all three panels, regions of lower signal-to-noise, and therefore less reliable temperature, are grayed out with a filter that gets more opaque toward lower signal-to-noise. The thin contours are from the Herschel HiGal dust SED fit at a level $N\left(\mathrm{H}_{2}\right)=5 \times 10^{22} \mathrm{~cm}^{-2}$ and are included to provide a visual reference for comparison between the temperature maps. 
A\&A 586, A50 (2016)

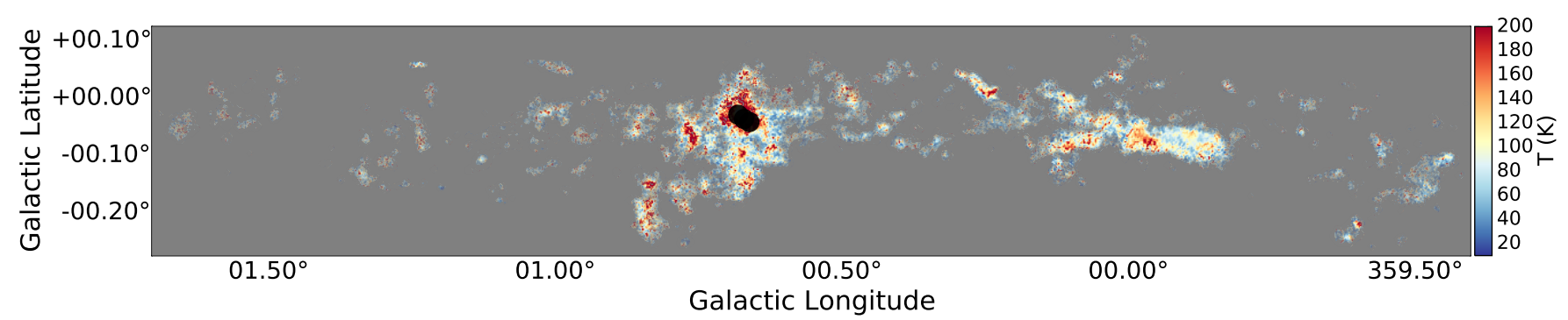

(a)

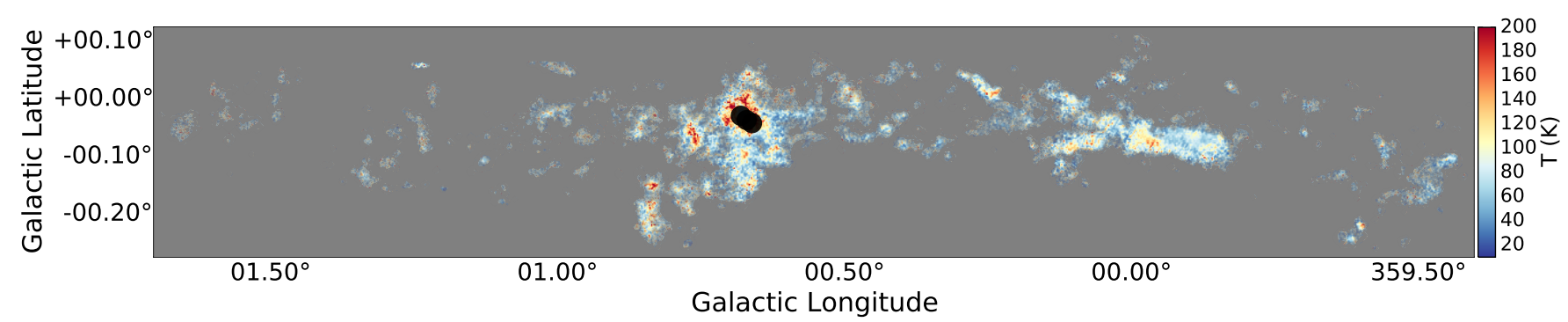

(b)

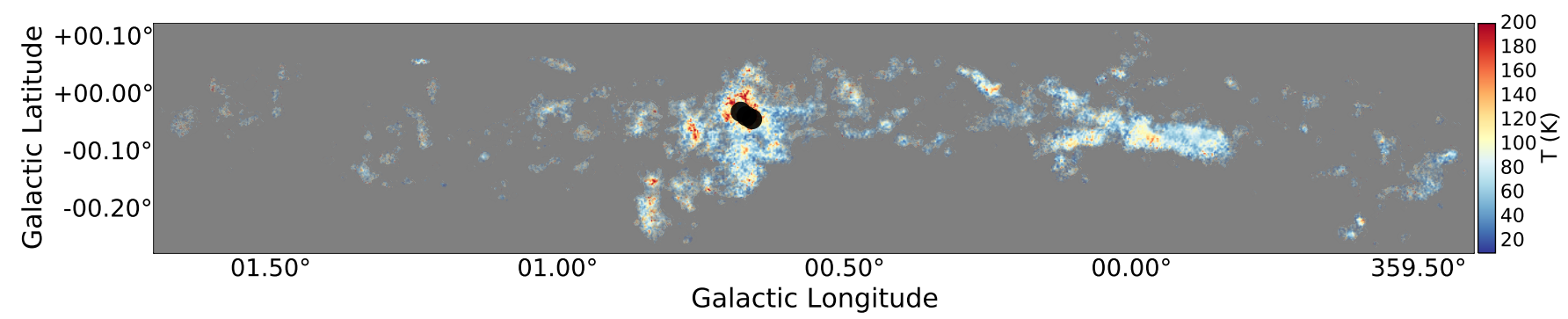

(c)

Fig. E.5. Temperature maps assuming fixed $X\left(\mathrm{p}-\mathrm{H}_{2} \mathrm{CO}\right)$ and $n\left(\mathrm{H}_{2}\right)=1 \times 10^{4} \mathrm{~cm}^{-3}$. The maps are masked by signal-to-noise as in Fig. 3. The thin black contours are at a column $N\left(\mathrm{H}_{2}\right)=5 \times 10^{22} \mathrm{~cm}^{-2}$ from the Herschel SED fit maps. a) $\left.X\left(\mathrm{p}-\mathrm{H}_{2} \mathrm{CO}\right)=1 \times 10^{-8}, \mathbf{b}\right) X\left(\mathrm{p}-\mathrm{H}_{2} \mathrm{CO}\right)=1.2 \times 10^{-9}$, c) $X\left(\mathrm{p}-\mathrm{H}_{2} \mathrm{CO}\right)=1 \times 10^{-10}$. 


\section{Appendix F: Temperature balance plots without data}

Figure 9 shows theoretical curves with all data points superposed. For clarity, we reproduce that figure with no data plotted here in Fig. F.1.

The legend shows the various modifications to model parameters used. The fiducial model is indicated by a dashed black line with $\zeta_{\mathrm{CR}}=1 \times 10^{-17} \mathrm{~s}^{-1}, n=10^{4} \mathrm{~cm}^{-3}, L=5 \mathrm{pc}$, velocity gradient $\mathrm{d} v / \mathrm{d} r=5 \mathrm{~km} \mathrm{~s}^{-1} \mathrm{pc}^{-1}$, dust temperature $T_{\mathrm{D}}=25 \mathrm{~K}$, dust radiation temperature $T_{\mathrm{D} \text {,rad }}=10 \mathrm{~K}$, and interstellar radiation field $G_{0}$. The green solid curve shows a model that follows both a size-linewidth relation $L=5 \sigma_{5}^{0.7}$ pc and is isobaric with $n=10^{4.25} \sigma_{5}^{2} \mathrm{~cm}^{-3}$, where $\sigma_{5}$ is the velocity dispersion in units of $5 \mathrm{~km} \mathrm{~s}^{-1}$. The green dashed curve shows the same with only the size-linewidth relation included. The green dotted curve shows a high cosmic-ray ionization rate with no turbulent heating and is meant to show the absolute floor temperature guaranteed by such a high CRIR.

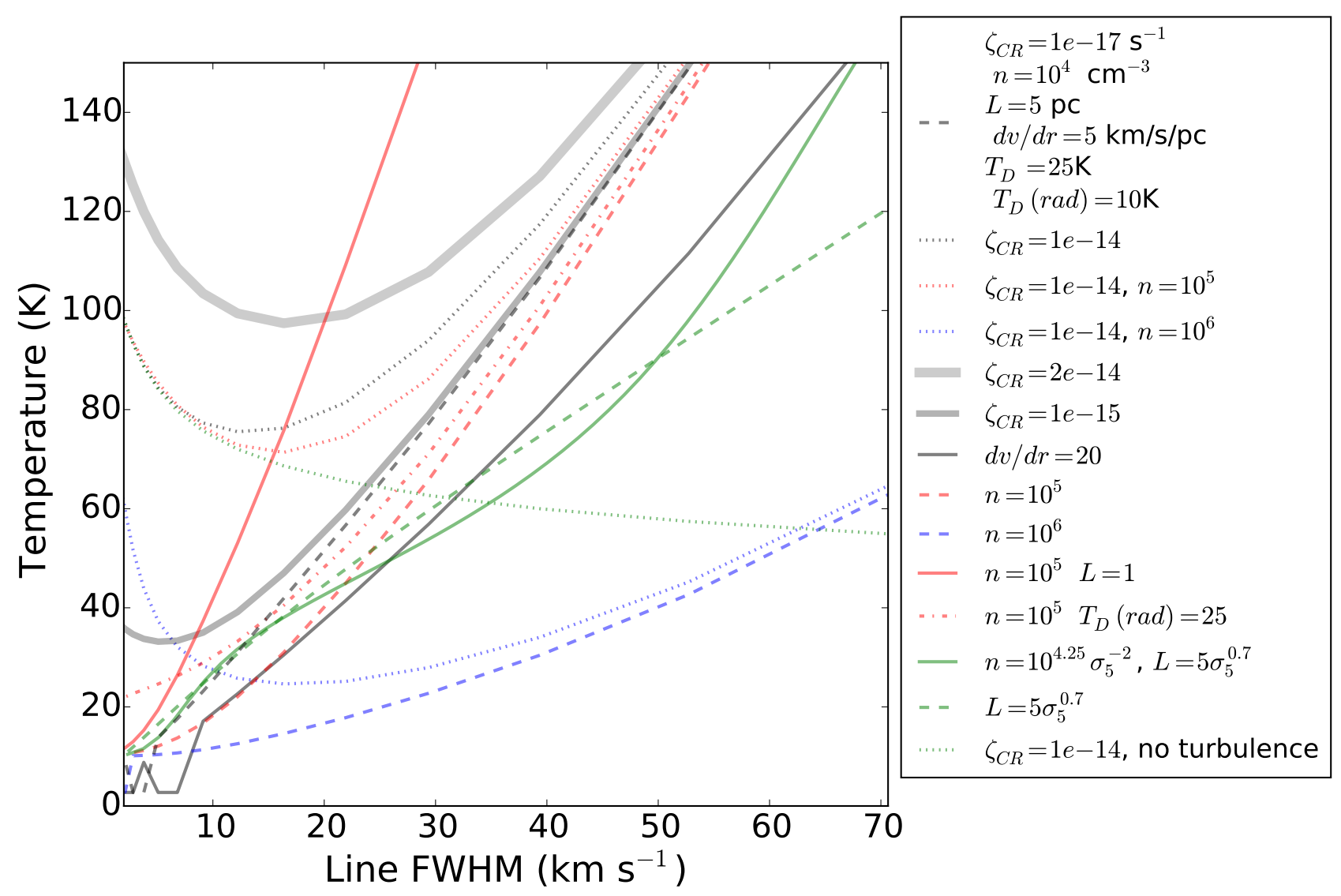

Fig. F.1. DESPOTIC-derived equilibrium temperature as a function of line width with no data overlaid. The curves are identical to Fig. 9. 


\section{Appendix G: Source table}

We include an excerpt from the source tables here. The full tables are available in digital form from https://raw.githubusercontent.com/adamginsburg/ APEX_CMZ_H2CO/v1.1/tables/PPV_H2CO_Temperature_ orbit.ipac and https://raw.githubusercontent.com/ adamginsburg/APEX_CMZ_H2CO/v1.1/tables/fitted_ line_parameters_Chi2Constraints_orbit.ipac.
Both tables are also available on the dataverse page. A complete description of the columns is available at https://github.com/adamginsburg/APEX_CMZ_H2CO/ blob/v1.1/tables/README.rst

Table G.1. $\mathrm{H}_{2} \mathrm{CO}$ line parameters and fit properties.

\begin{tabular}{|c|c|c|c|c|c|c|c|}
\hline Source name & $R_{1}$ & $\begin{array}{c}\sigma_{v} \\
\mathrm{~km} \mathrm{~s}^{-1}\end{array}$ & $\begin{array}{c}v_{\mathrm{lsr}} \\
\mathrm{km} \mathrm{s}^{-1}\end{array}$ & $\begin{array}{c}T_{\mathrm{B}}\left(\mathrm{H}_{2} \mathrm{CO}\right) \\
\mathrm{K}\end{array}$ & $\log \left(n\left(\mathrm{H}_{2}\right)\right)$ & $\begin{array}{c}T_{\text {gas }} \\
\mathrm{K}\end{array}$ & $\begin{array}{c}T_{\text {gas,turb }} \\
\mathrm{K}\end{array}$ \\
\hline Menten1 & $0.35 \pm 0.03$ & $4.93 \pm 0.15$ & $55.85 \pm 0.15$ & $1.65 \pm 0.05$ & 22.8 & $83.55_{-44.41}^{+16.65}$ & 36.8 \\
\hline Menten7 & $0.76 \pm 0.10$ & $10.34 \pm 0.97$ & $54.41 \pm 0.98$ & $0.35 \pm 0.04$ & 22.4 & $350.00_{-5.55}^{+8.1 .88}$ & 92.6 \\
\hline dn:G0.41+0.05box & $0.29 \pm 0.02$ & $11.19 \pm 0.27$ & $19.59 \pm 0.27$ & $1.55 \pm 0.04$ & 23.1 & $66.90_{-18.04}^{+11.10}$ & 102 \\
\hline $20 \mathrm{kms}$ & $0.32 \pm 0.00$ & $8.15 \pm 0.02$ & $17.18 \pm 0.02$ & $12.39 \pm 0.03$ & 23.3 & $62.73_{-20.82}^{+12.49}$ & 34.7 \\
\hline CoolSpot & $0.22 \pm 0.02$ & $7.26 \pm 0.39$ & $27.60 \pm 0.56$ & $3.79 \pm 0.13$ & 22.9 & $48.86_{-6.94}^{+11.10}$ & 140 \\
\hline G1.34-0.13 & $0.32 \pm 0.02$ & $5.19 \pm 0.16$ & $32.27 \pm 0.15$ & $1.68 \pm 0.05$ & 22.7 & $75.22_{-29.14}^{+12.49}$ & 41.3 \\
\hline G0.43-0.05 & $0.29 \pm 0.01$ & $10.25 \pm 0.13$ & $89.93 \pm 0.14$ & $2.84 \pm 0.03$ & 22.5 & $66.90_{-15.27}^{+9.71}$ & 87 \\
\hline G359.49-0.14 & $0.25 \pm 0.02$ & $9.82 \pm 0.20$ & $-56.04 \pm 0.20$ & $1.57 \pm 0.03$ & 22.8 & $55.80_{-9.71}^{+8.33}$ & 58.1 \\
\hline G0.76-0.08 & $0.76 \pm 0.05$ & $12.49 \pm 0.35$ & $19.83 \pm 0.26$ & $1.62 \pm 0.10$ & 23.1 & $350.00_{-5.55}^{+38.86}$ & 131 \\
\hline G0.24-0.05box & $0.20 \pm 0.02$ & $7.50 \pm 0.18$ & $79.72 \pm 0.18$ & $1.31 \pm 0.03$ & 22.7 & $46.08_{-6.94}^{+6.94}$ & 56.7 \\
\hline G0.85-0.04box & $0.31 \pm 0.01$ & $17.99 \pm 0.26$ & $4.03 \pm 0.27$ & $1.16 \pm 0.02$ & 22.9 & $73.84_{-23.59}^{+8.33}$ & 129 \\
\hline G359.8_box & $0.23 \pm 0.01$ & $10.53 \pm 0.25$ & $11.55 \pm 0.17$ & $0.72 \pm 0.02$ & 22.5 & $53.02_{-8.33}^{+5.55}$ & 47.5 \\
\hline G0.83-0.19box & $0.36 \pm 0.01$ & $10.11 \pm 0.14$ & $43.97 \pm 0.14$ & $1.76 \pm 0.03$ & 22.9 & $89.10_{-43.02}^{+9.71}$ & 50.3 \\
\hline Map_002 Off 2 & $0.18 \pm 0.04$ & $11.23 \pm 1.33$ & $6.48 \pm 1.54$ & $0.22 \pm 0.02$ & 22.8 & $46.08_{-12.49}^{+15.27}$ & 66.6 \\
\hline Map_007 Off 2 & $0.22 \pm 0.01$ & $26.86 \pm 0.22$ & $45.78 \pm 0.27$ & $1.30 \pm 0.01$ & 23.2 & $51.63_{-6.94}^{+8.33}$ & 142 \\
\hline Map_012 Off 2 & $0.31 \pm 0.06$ & $9.83 \pm 1.17$ & $7.63 \pm 1.34$ & $0.24 \pm 0.02$ & 22.5 & $79.39_{-51.35}^{+27.76}$ & 59.2 \\
\hline Map_023 Off 3 & $0.08 \pm 0.04$ & $19.67 \pm 1.30$ & $33.04 \pm 1.25$ & $0.28 \pm 0.01$ & 22.5 & $28.04_{-5.55}^{+9.71}$ & 114 \\
\hline Map_027 Off 2 & $0.15 \pm 0.03$ & $10.86 \pm 0.45$ & $81.72 \pm 0.43$ & $0.52 \pm 0.02$ & 22.8 & $37.76_{-5.55}^{+8.33}$ & 51.3 \\
\hline Map_055 Off 3 & $0.27 \pm 0.02$ & $8.36 \pm 0.27$ & $10.94 \pm 0.20$ & $0.81 \pm 0.03$ & 22.8 & $62.73_{-15.27}^{+9.71}$ & 47 \\
\hline Map_116 Off 1 & $0.33 \pm 0.03$ & $13.42 \pm 0.53$ & $64.31 \pm 0.55$ & $0.41 \pm 0.02$ & 22.5 & $80.78_{-37.47}^{+16.65}$ & 79.3 \\
\hline Map_122 Off 1 & $0.09 \pm 0.05$ & $22.05 \pm 1.61$ & $10.11 \pm 1.55$ & $0.19 \pm 0.01$ & 22.7 & $30.82_{-6.94}^{+12.49}$ & 128 \\
\hline
\end{tabular}




\section{A. Ginsburg et al.: APEX CMZ $\mathrm{H}_{2} \mathrm{CO}$}

Table G.2. $\mathrm{H}_{2} \mathrm{CO}$ parameters and fit properties for dendrogram-selected clumps.

\begin{tabular}{|c|c|c|c|c|c|c|c|}
\hline Source ID & $R_{1}$ & $\begin{array}{c}\sigma_{v} \\
\mathrm{~km} \mathrm{~s}^{-1}\end{array}$ & $\begin{array}{c}v_{\mathrm{lsr}} \\
\mathrm{km} \mathrm{s}^{-1}\end{array}$ & $\begin{array}{c}\operatorname{Max} T_{\mathrm{B}}\left(3_{0,3}\right) \\
\mathrm{K}\end{array}$ & $\log \left(n\left(\mathrm{H}_{2}\right)\right)$ & $\begin{array}{c}T_{\text {gas }} \\
\mathrm{K}\end{array}$ & $\begin{array}{c}T_{\text {gas,turb }} \\
\text { K }\end{array}$ \\
\hline 0 & $0.127 \pm 0.022$ & 0.00211 & -111 & 0.513 & 22.3 & $34.98_{-4.16}^{+5.55}$ & 35 \\
\hline 10 & $0.206 \pm 0.010$ & 0.0033 & -57.7 & 0.525 & 22.8 & $50.24_{-4.16}^{+6.94}$ & 56 \\
\hline 20 & $0.055 \pm 0.026$ & 0.00251 & -38 & 0.474 & 22.7 & $23.88_{-1.39}^{+6.16}$ & 59 \\
\hline 30 & $0.245 \pm 0.006$ & 0.00744 & -15 & 0.683 & 22.7 & $59.96_{-6.94}^{-1.399}$ & 118 \\
\hline 40 & $0.322 \pm 0.000$ & 0.019 & 27.7 & 2.3 & 23.1 & $79.39_{-2637}^{+8.33}$ & 122 \\
\hline 50 & $0.332 \pm 0.002$ & 0.0141 & 28.2 & 0.99 & 23.1 & $86.33_{-2359}^{+9.71}$ & 147 \\
\hline 60 & $0.317 \pm 0.001$ & 0.00886 & 14.7 & 2.3 & 23.3 & $78.00_{-20.82}^{+11.10}$ & 88 \\
\hline 70 & $0.382 \pm 0.001$ & 0.0172 & 47.4 & 3.97 & 23.3 & $98.82_{-68.00}^{+11.82}$ & 76 \\
\hline 80 & $0.420 \pm 0.002$ & 0.0116 & 25 & 0.604 & 23 & $121.02_{-116.57}^{+8.30}$ & 155 \\
\hline 90 & $0.382 \pm 0.001$ & 0.0172 & 47.4 & 3.97 & 23.3 & $105.76_{-63.84}^{+18.04}$ & 78 \\
\hline 100 & $0.235 \pm 0.014$ & 0.00394 & 11.4 & 0.411 & 23 & $54.41_{-8.33}^{+5.55}$ & 118 \\
\hline 110 & $0.460 \pm 0.001$ & 0.016 & 49 & 3.97 & 23.6 & $140.45_{-215.10}^{+2.78}$ & 104 \\
\hline 120 & $0.289 \pm 0.003$ & 0.00591 & 31.3 & 0.883 & 23.1 & $69.67_{-16.65}^{+5.55}$ & 47 \\
\hline 130 & $0.222 \pm 0.005$ & 0.00512 & 28.5 & 0.82 & 22.6 & $53.02_{-6.94}^{-1.05}$ & 54 \\
\hline 140 & $0.089 \pm 0.018$ & 0.00371 & 28.1 & 0.527 & 22.6 & $29.43_{-1.39}^{+5.54}$ & 107 \\
\hline 150 & $0.257 \pm 0.014$ & 0.0051 & 31.8 & 0.469 & 22.7 & $64.12_{-6.94}^{+9.39}$ & 128 \\
\hline 160 & $0.311 \pm 0.009$ & 0.00281 & 29.2 & 0.816 & 23.1 & $\begin{array}{l}72.45_{-23.59}^{+5.55} \\
0\end{array}$ & 145 \\
\hline 170 & $0.353 \pm 0.003$ & 0.00576 & 45 & 0.819 & 23 & $91.88_{-37.47}^{+6.94}$ & 56 \\
\hline 180 & $0.318 \pm 0.003$ & 0.00261 & 29.9 & 1.16 & 23.2 & $75.22_{-26.37}^{+4.41}$ & 88 \\
\hline 190 & $0.311 \pm 0.001$ & 0.00734 & 50.7 & 1.72 & 22.8 & $73.84_{-24.98}^{+4.36}$ & 37 \\
\hline 200 & $0.321 \pm 0.001$ & 0.00706 & 50.6 & 1.72 & 22.8 & $76.61_{-29.14}^{+2.78}$ & 38 \\
\hline 210 & $0.351 \pm 0.003$ & 0.0061 & 49.7 & 1.27 & 23.1 & $86.33_{-40.24}^{+4.16}$ & 138 \\
\hline 220 & $0.161 \pm 0.022$ & 0.00306 & 46.4 & 0.416 & 23 & $40.53_{-4.16}^{+6.94}$ & 82 \\
\hline 230 & $0.215 \pm 0.015$ & 0.00261 & 47.4 & 0.405 & 22.9 & $53.02_{-4.16}^{-4.16}$ & 57 \\
\hline 240 & $0.265 \pm 0.009$ & 0.00225 & 49.4 & 0.626 & 22.6 & $61.35_{-12.49}^{+4.16}$ & 75 \\
\hline 250 & $0.313 \pm 0.021$ & 0.00435 & 58 & 0.418 & 23.1 & $75.22_{-22.20}^{+11.49}$ & 93 \\
\hline 260 & $0.190 \pm 0.015$ & 0.00473 & 59.6 & 0.433 & 22.7 & $48.86_{-2.78}^{+8.33}$ & 98 \\
\hline 270 & $0.261 \pm 0.007$ & 0.0042 & 65.2 & 0.827 & 22.7 & $61.35_{-11.10}^{+4.16}$ & 74 \\
\hline 280 & $0.653 \pm 0.007$ & 0.00284 & 70.7 & 1 & 24 & $350.00_{-5.55}^{+1.39}$ & 85 \\
\hline 290 & $0.220 \pm 0.009$ & 0.0044 & 79.9 & 0.373 & 23 & $55.80_{-2.78}^{+9.71}$ & 80 \\
\hline 300 & $0.242 \pm 0.020$ & 0.00357 & 80.2 & 0.359 & 23.1 & $55.80_{-9.71}^{+8.33}$ & 93 \\
\hline 310 & $0.246 \pm 0.012$ & 0.00331 & 82.7 & 0.446 & 22.9 & $62.73_{-4.16}^{+11.10}$ & 57 \\
\hline 320 & $0.251 \pm 0.023$ & 0.00295 & 85.9 & 0.456 & 23.1 & $57.18_{-12.49}^{+8.33}$ & 69 \\
\hline 330 & $0.140 \pm 0.028$ & 0.00247 & 90 & 0.371 & 22.9 & $37.76_{-4.16}^{+8.33}$ & 61 \\
\hline 340 & $0.054 \pm 0.028$ & 0.00345 & 97.1 & 0.532 & 22.6 & $22.49_{-2.78}^{+6.94}$ & 70 \\
\hline 350 & $0.164 \pm 0.014$ & 0.00334 & 117 & 0.504 & 22.7 & $43.31_{-1.39}^{+6.94}$ & 59 \\
\hline
\end{tabular}

